\title{
The $B\left(C_{6} F_{5}\right)_{3}$-Catalyzed Tandem Meinwald- Rearrangement Reductive Amination
}

Martine R. Tiddens, Robertus J. M. Klein Gebbink and Matthias Otte*

Organic Chemistry \& Catalysis, Debye Institute for Nanomaterials Science, Universiteit

Utrecht, Universiteitsweg 99, 3584 CG, Utrecht, The Netherlands

\section{Supporting Information}

Table of contents

1. General information S1

2. Experiments $\quad \mathrm{S} 2$

3. References $\quad \mathrm{S} 16$

4. Spectra $\quad \mathrm{S} 17$

\section{General information}

All reactions involving air- or moisture sensitive compounds were carried out under Nitrogen using standard Schlenk- and vacuum line techniques. All reagents were purchased from commercial sources and used as received unless stated otherwise. Dichloromethane $\left(\mathrm{CH}_{2} \mathrm{Cl}_{2}\right)$ and methanol $(\mathrm{MeOH})$ were distilled over calcium hydride. Chlorobenzene ( $\mathrm{PhCl})$, 1,2-dichloroethane (1,2-DCE), toluene and 1,4dioxane (for Method A) were used as supplied from commercial sources. 1,4-Dioxane (for Method B) was distilled over sodium/benzophenone. Diethyl ether and toluene used for the synthesis of $\mathrm{B}(p \text {-tol })_{3}$ were taken from a MBRAUN MB SPS-80 solvent purification system. Compounds 1a, 1f, 1g, 1h, 2a, 2b, 2c, 2d, 2e, 2f, 2g, 2h, 2i, 2j, 2k, 2l, 2m, 4, S1, S2, S4, S6, S8, S10 and $\mathrm{B}\left(\mathrm{C}_{6} \mathrm{~F}_{5}\right)_{3}$ are commercially available and have been used without further purification.

${ }^{1} \mathrm{H}$ - and ${ }^{13} \mathrm{C}-\mathrm{NMR}$ spectra (400 and $100 \mathrm{MHz}$, respectively) were recorded on an Agilent MRF400 or Varian VNMR-S400 spectrometer at $25^{\circ} \mathrm{C}$. ${ }^{1} \mathrm{H}-$ and ${ }^{13} \mathrm{C}-\mathrm{NMR}$ chemical shifts are reported in ppm relative to TMS using the residual solvent resonance as internal standard.

Infrared spectra were recorded using a Perkin Elmer Spectrum One FT-IR spectrometer. MS measurements were performed on a Waters LCT Premier XE KE317. Samples were prepared by solving the products in dichloromethane, acetonitrile and/or water. Where necessary, reagents such as formic acid, silver nitrate or pyridine were added in order to obtain ionization. 


\section{Experiments}

\subsection{General procedure}

General procedure 1: $\mathrm{B}\left(\mathrm{C}_{6} \mathrm{~F}_{5}\right)_{3}$-catalyzed tandem Meinwald-rearrangement reductive amination

Method A: A flame dried Schlenk-flask equipped with a stirring bean was taken into a GloveBox. The Lewis-acid $\left.\left(\mathrm{B}_{(} \mathrm{C}_{6} \mathrm{~F}_{5}\right)_{3}, 5 \mathrm{~mol} \%\right)$ was added to the Schlenk-flask. The flask was taken out of the GloveBox and connected to a Schlenk-line. The epoxide (0.5 mmol, 1.0 equiv.) solved in $\mathrm{PhCl}(2 \mathrm{~mL})$ was added to the Lewis-acid. The mixture was allowed to stir at room temperature for $10 \mathrm{~min}$. The amine (1.2 equiv.) solved in $\mathrm{PhCl}(1 \mathrm{~mL})$ was added to the reaction mixture. The mixture was allowed to stir at room temperature for $5 \mathrm{~min}$. $\mathrm{PhMe}_{2} \mathrm{SiH}$ (1.2 equiv.) solved in $\mathrm{PhCl}(1 \mathrm{~mL})$ was added to the reaction mixture. After addition, the Schlenk-flask was immediately put into an oil bath at $100{ }^{\circ} \mathrm{C}$. The mixture was stirred for $3 \mathrm{~h}$ at $100{ }^{\circ} \mathrm{C}$ oil bath temperature. The mixture was allowed to cool to room temperature. The solvent was removed under vacuum at $60{ }^{\circ} \mathrm{C}$. Products that have not been described in literature and 3a were purified via flash column chromatography. Yields of products described in literature were determined via NMR by making use of an internal standard.

Method B: A flame dried Schlenk-flask equipped with a stirring bean was taken into

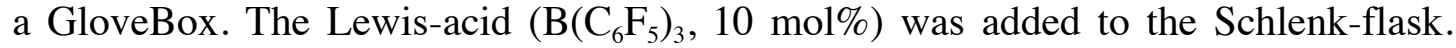
The flask was taken out of the GloveBox and connected to a Schlenk-line. The epoxide $(0.5 \mathrm{mmol}, 1.0$ equiv.) solved in 1,4-dioxane $(0.5 \mathrm{~mL})$ was added to the Lewis-acid. The mixture was allowed to stir at room temperature for $10 \mathrm{~min}$. The amine (1.2 equiv.) solved in $\mathrm{PhCl}(2.5 \mathrm{~mL})$ was added to the reaction mixture. The mixture was allowed to stir at room temperature for $5 \mathrm{~min}$. $\mathrm{PhMe}_{2} \mathrm{SiH}$ (1.2 equiv.) solved in $\mathrm{PhCl}(1 \mathrm{~mL})$ was added to the reaction mixture. After addition the Schlenkflask was immediately put into an oil bath at $100{ }^{\circ} \mathrm{C}$. The mixture was stirred for $3 \mathrm{~h}$ at $100{ }^{\circ} \mathrm{C}$ oil bath temperature. The mixture was allowed to cool to room temperature. The solvent was removed under vacuum at $60{ }^{\circ} \mathrm{C}$. Products that have not been described in literature were purified via flash column chromatography. Yields of products described in literature were determined via NMR by making use of an internal standard.

\section{General procedure 2: Wittig reaction}

In a flame dried three-neck flask under $\mathrm{N}_{2}$ were placed $\mathbf{S 1}(4.33 \mathrm{~g}, 10.0 \mathrm{mmol})$ and 18-crown-6 (264 mg, $1.00 \mathrm{mmol})$. Freshly distilled $\mathrm{CH}_{2} \mathrm{Cl}_{2}(50 \mathrm{~mL})$ was added and the mixture was cooled to $-79{ }^{\circ} \mathrm{C}$. The aldehyde $(11.0 \mathrm{mmol}, 1.1$ equiv.) and $\mathrm{KOH}$ $(1.20 \mathrm{~g}, 21.5 \mathrm{mmol})$ were added to the mixture. The mixture was allowed to warm slowly up to room temperature and to stir at room temperature overnight. The reaction mixture was filtered and the residue was washed with $\mathrm{CH}_{2} \mathrm{Cl}_{2}$. The combined organics were washed with water and dried over $\mathrm{MgSO}_{4}$. After removing of the solvent, the crude product was purified via flash column chromatography. 
General procedure 3: Epoxidation with $m \mathrm{CPBA}$

The olefin (1.0 equiv.) was solved in distilled $\mathrm{CH}_{2} \mathrm{Cl}_{2}$. The mixture was cooled to 0 ${ }^{\circ} \mathrm{C}$. mCPBA (70-75\% in water, $340 \mathrm{mg}$ per mmol olefin) was added to the reaction mixture. After warming to room temperature the mixture was stirred overnight at room temperature. Saturated $\mathrm{NaHCO}_{3}$ aq. was added to the reaction mixture. After phase separation the aqueous phase was extracted with $\mathrm{Et}_{2} \mathrm{O}$. The combined organics were washed with brine and dried over $\mathrm{MgSO}_{4}$. After removing of the solvent, the crude product was purified via flash column chromatography.

2.2. Optimization experiments for the $\mathrm{B}\left(\mathrm{C}_{6} \mathrm{~F}_{5}\right)_{3}$-catalyzed tandem Meinwaldrearrangement reductive amination - Synthesis of $\mathbf{3 a}$<smiles>c1ccc(NCC(c2ccccc2)c2ccccc2)cc1</smiles>

Entry 1: According to general procedure 1 (Method A), 1a (96 mg, $0.5 \mathrm{mmol}, 1.0$ equiv.), $\mathrm{B}\left(\mathrm{C}_{6} \mathrm{~F}_{5}\right)_{3}(12.5 \mathrm{mg}, 0.024 \mathrm{mmol}, 0.05$ equiv.), $2 \mathrm{a}$ (92 $\mathrm{mg}, 1.00 \mathrm{mmol}, 2.0$ equiv.) and $\mathrm{PhMe}_{2} \mathrm{SiH}$ (136 mg, $1.00 \mathrm{mmol}, 2.0$ equiv.) were stirred in $\mathrm{PhCl}(4 \mathrm{~mL})$ for $3 \mathrm{~h}$ at $100{ }^{\circ} \mathrm{C}$. After flash column chromatography (PE:EtOAc $\left.=95: 5, \mathrm{R}_{F}=0.10\right)$ 3a was obtained as yellowish oil (117 mg, $0.43 \mathrm{mmol}, 86 \%)$. ${ }^{1} \mathrm{H}-\mathrm{NMR}(400 \mathrm{MHz}$, $\left.\mathrm{CDCl}_{3}\right): 7.34-7.22(\mathrm{~m}, 10 \mathrm{H}), 7.17(\mathrm{t}, J=7.9 \mathrm{~Hz}, 2 \mathrm{H}), 6.71(\mathrm{t}, J=7.3 \mathrm{~Hz}, 1 \mathrm{H}), 6.59$ $(\mathrm{d}, J=8.5 \mathrm{~Hz}, 2 \mathrm{H}), 4.30(\mathrm{t}, J=7.7 \mathrm{~Hz}, 1 \mathrm{H}), 3.74(\mathrm{~d}, J=7.6 \mathrm{~Hz}, 2 \mathrm{H}), 3.63(\mathrm{br}, 1 \mathrm{H})$. ${ }^{13} \mathrm{C}-\mathrm{NMR}\left(100 \mathrm{MHz}, \mathrm{CDCl}_{3}\right)$ : 147.78, 142.23, 129.28, 128.71, 128.12, 128.76, $117.63,113.12,50.24,48.51$. NMR data matches with those reported in literature. ${ }^{[\mathrm{S} 1]}$

Entry 2: According to general procedure 1 (Method A), 1a (96 mg, $0.5 \mathrm{mmol}, 1.0$ equiv.), 2a (92 mg, $1.00 \mathrm{mmol}, 2.0$ equiv.) and $\mathrm{PhMe}_{2} \mathrm{SiH}$ (136 mg, $1.0 \mathrm{mmol}, 2.0$ equiv.) were stirred in $\mathrm{PhCl}(4 \mathrm{~mL})$ for $3 \mathrm{~h}$ at $100{ }^{\circ} \mathrm{C}$. NMR analysis of the crude reaction mixture indicated no formation of $\mathbf{3 a}$ and no or only little conversion of $\mathbf{1 a}$.

Entry 3: According to general procedure 1 (Method A), 1a (92 mg, $0.48 \mathrm{mmol}, 1.0$ equiv.), $\mathrm{B}\left(\mathrm{C}_{6} \mathrm{~F}_{5}\right)_{3}(11.5 \mathrm{mg}, 0.022 \mathrm{mmol}, 0.05$ equiv.), $2 \mathrm{a}$ (56 mg, $0.60 \mathrm{mmol}, 1.2$ equiv.) and $\mathrm{PhMe}{ }_{2} \mathrm{SiH}(81 \mathrm{mg}, 0.60 \mathrm{mmol}, 1.2$ equiv.) were stirred in $\mathrm{PhCl}(4 \mathrm{~mL})$ for $3 \mathrm{~h}$ at $100{ }^{\circ} \mathrm{C}$. After flash column chromatography (PE:EtOAc $\left.=95: 5\right)$ 3a was obtained as yellowish oil (114 $\mathrm{mg}, 0.42 \mathrm{mmol}, 88 \%)$.

Entry 4: According to general procedure 1 (Method A), 1a (92 mg, $0.48 \mathrm{mmol}, 1.0$ equiv.), $\mathrm{B}\left(\mathrm{C}_{6} \mathrm{~F}_{5}\right)_{3}(13.0 \mathrm{mg}, 0.025 \mathrm{mmol}, 0.05$ equiv.), $2 \mathrm{a}$ (93 $\mathrm{mg}, 1.00 \mathrm{mmol}, 2.0$ equiv.) and $\mathrm{PhMe}_{2} \mathrm{SiH}$ (136 mg, $1.00 \mathrm{mmol}, 2.0$ equiv.) were stirred in $\mathrm{PhCl}(4 \mathrm{~mL})$ for $6 \mathrm{~h}$ at $100{ }^{\circ} \mathrm{C}$. After flash column chromatography $(\mathrm{PE}:$ EtOAc $=95: 5)$ 3a was obtained as yellowish oil (114 mg, $0.42 \mathrm{mmol}, 88 \%)$.

Entry 5: According to general procedure 1 (Method A), 1a (96 mg, $0.50 \mathrm{mmol}, 1.0$ equiv.), $\mathrm{B}\left(\mathrm{C}_{6} \mathrm{~F}_{5}\right)_{3}$ (6.2 $\mathrm{mg}, 0.012 \mathrm{mmol}, 0.024$ equiv.), $2 \mathrm{a}$ (56 mg, $0.60 \mathrm{mmol}, 1.2$ 
equiv.) and $\mathrm{PhMe}_{2} \mathrm{SiH}(81 \mathrm{mg}, 0.60 \mathrm{mmol}, 1.2$ equiv.) were stirred in $\mathrm{PhCl}(4 \mathrm{~mL})$ for $3 \mathrm{~h}$ at $100{ }^{\circ} \mathrm{C}$. After flash column chromatography (PE:EtOAc $\left.=95: 5\right)$ 3a was obtained as yellowish oil (108 mg, $0.39 \mathrm{mmol}, 79 \%)$.

Entry 6: According to general procedure 1 (Method A), 1a (96 mg, $0.50 \mathrm{mmol}, 1.0$ equiv.), $\mathrm{B}\left(\mathrm{C}_{6} \mathrm{~F}_{5}\right)_{3}(12.5 \mathrm{mg}, 0.024 \mathrm{mmol}, 0.05$ equiv.), $2 \mathrm{a}(56 \mathrm{mg}, 0.60 \mathrm{mmol}, 1.2$ equiv.) and $\mathrm{PhMe}_{2} \mathrm{SiH}$ (81 mg, $0.60 \mathrm{mmol}, 1.2$ equiv.) were stirred in $\mathrm{PhCl}(4 \mathrm{~mL})$ for $3 \mathrm{~h}$ at $60{ }^{\circ} \mathrm{C}$. After flash column chromatography (PE:EtOAc $\left.=95: 5\right)$ 3a was obtained as yellowish oil (20 mg, $0.07 \mathrm{mmol}, 15 \%)$.

Entry 7: According to general procedure 1 (Method A), 1a (96 mg, $0.50 \mathrm{mmol}, 1.0$ equiv.), $\mathrm{B}\left(\mathrm{C}_{6} \mathrm{~F}_{5}\right)_{3}(12.5 \mathrm{mg}, 0.024 \mathrm{mmol}, 0.05$ equiv.), $2 \mathrm{a}(56 \mathrm{mg}, 0.60 \mathrm{mmol}, 1.2$ equiv.) and $\mathrm{PhMe}_{2} \mathrm{SiH}(81 \mathrm{mg}, 0.60 \mathrm{mmol}, 1.2$ equiv.) were stirred in 1,2dichloroethane $(4 \mathrm{~mL})$ for $3 \mathrm{~h}$ at $100{ }^{\circ} \mathrm{C}$. After flash column chromatography $(\mathrm{PE}: \mathrm{EtOAc}=95: 5)$ 3a was obtained as yellowish oil $(110 \mathrm{mg}, 0.40 \mathrm{mmol}, 80 \%)$.

Entry 8: According to general procedure 1 (Method A), 1a (96 mg, $0.50 \mathrm{mmol}, 1.0$ equiv.), $\mathrm{B}\left(\mathrm{C}_{6} \mathrm{~F}_{5}\right)_{3}(12.5 \mathrm{mg}, 0.024 \mathrm{mmol}, 0.05$ equiv.), $2 \mathrm{a}(56 \mathrm{mg}, 0.60 \mathrm{mmol}, 1.2$ equiv.) and $\mathrm{PhMe}_{2} \mathrm{SiH}(81 \mathrm{mg}, 0.60 \mathrm{mmol}, 1.2$ equiv.) were stirred in toluene (4 mL) for $3 \mathrm{~h}$ at $100{ }^{\circ} \mathrm{C}$. After flash column chromatography (PE:EtOAc $\left.=95: 5\right)$ 3a was obtained as yellowish oil (104 $\mathrm{mg}, 0.38 \mathrm{mmol}, 76 \%$ ).

Entry 9: According to general procedure 1 (Method A), 1a (96 mg, $0.50 \mathrm{mmol}, 1.0$ equiv.), $\mathrm{B}\left(\mathrm{C}_{6} \mathrm{~F}_{5}\right)_{3}(12.5 \mathrm{mg}, 0.024 \mathrm{mmol}, 0.05$ equiv.), $2 \mathrm{a}(56 \mathrm{mg}, 0.60 \mathrm{mmol}, 1.2$ equiv.) and $\mathrm{PhMe}_{2} \mathrm{SiH}$ (81 mg, $0.60 \mathrm{mmol}, 1.2$ equiv.) were stirred in 1,4-dioxane (4 $\mathrm{mL})$ for $3 \mathrm{~h}$ at $100{ }^{\circ} \mathrm{C}$. After flash column chromatography (PE:EtOAc $\left.=95: 5\right)$ 3a was obtained as yellowish oil ( $28 \mathrm{mg}, 0.11 \mathrm{mmol}, 21 \%)$.

Entry 10: According to general procedure 1 (Method A), $1 \mathbf{a}$ (96 mg, $0.50 \mathrm{mmol}, 1.0$ equiv.), $\mathrm{B}(p \text {-tol })_{3}(6.6 \mathrm{mg}, 0.023 \mathrm{mmol}, 0.05$ equiv.), $2 \mathrm{a}$ (56 $\mathrm{mg}, 0.60 \mathrm{mmol}, 1.2$ equiv.) and $\mathrm{PhMe}_{2} \mathrm{SiH}(81 \mathrm{mg}, 0.60 \mathrm{mmol}, 1.2$ equiv.) were stirred in $\mathrm{PhCl}(4 \mathrm{~mL})$ for $3 \mathrm{~h}$ at $100{ }^{\circ} \mathrm{C}$. NMR analysis of the crude reaction mixture indicated no formation of 3a.

Entry 11: According to general procedure 1 (Method A), $1 \mathbf{a}$ (96 mg, $0.50 \mathrm{mmol}, 1.0$ equiv.), $\mathrm{B}\left(\mathrm{C}_{6} \mathrm{~F}_{5}\right)_{2}(\mathrm{Mes})(11.6 \mathrm{mg}, 0.025 \mathrm{mmol}, 0.05$ equiv.), $2 \mathbf{a}(56 \mathrm{mg}, 0.60 \mathrm{mmol}$, 1.2 equiv.) and $\mathrm{PhMe} \mathrm{SiH}_{2} \mathrm{SiH} \mathrm{mg}, 0.60 \mathrm{mmol}, 1.2$ equiv.) were stirred in $\mathrm{PhCl}(4 \mathrm{~mL})$ for $3 \mathrm{~h}$ at $60{ }^{\circ} \mathrm{C}$. After flash column chromatography (PE:EtOAc $\left.=95: 5\right)$ 3a was obtained as yellowish oil (14 mg, $0.05 \mathrm{mmol}, 10 \%)$.

2.3. Synthesis of $\mathbf{3 b}$<smiles>COc1ccc(NCC(c2ccccc2)c2ccccc2)cc1</smiles>

$3 b$ 
According to general procedure 1 (Method A), $1 \mathrm{a}(98 \mathrm{mg}, 0.50 \mathrm{mmol}, 1.0$ equiv.), $\mathrm{B}\left(\mathrm{C}_{6} \mathrm{~F}_{5}\right)_{3}$ (12.8 mg, $0.025 \mathrm{mmol}, 0.05$ equiv.), $2 \mathbf{b}$ (( $p$-Anisidine) $74 \mathrm{mg}, 0.60 \mathrm{mmol}$, 1.2 equiv.) and $\mathrm{PhMe}_{2} \mathrm{SiH}$ (82 mg, $0.60 \mathrm{mmol}, 1.2$ equiv.) were stirred in $\mathrm{PhCl}(4 \mathrm{~mL})$ for $3 \mathrm{~h}$ at $100{ }^{\circ} \mathrm{C}$. After flash column chromatography $\left(\mathrm{PE}:\right.$ EtOAc $=95: 5 ; \mathrm{R}_{F}=0.11$ ) 3b was obtained as green oil (121 mg, $0.40 \mathrm{mmol}, 80 \%)$. $\mathrm{PhMe}_{2} \mathrm{SiOSiMe}_{2} \mathrm{Ph}$ was identified as impurity. ${ }^{1} \mathrm{H}-\mathrm{NMR}\left(400 \mathrm{MHz}, \mathrm{CDCl}_{3}\right): 7.35-7.25(\mathrm{~m}, 10 \mathrm{H}), 6.76(\mathrm{~d}, J=$ $8.8 \mathrm{~Hz}, 2 \mathrm{H}), 6.55(\mathrm{~d}, J=8.8 \mathrm{~Hz}, 2 \mathrm{H}), 4.28(\mathrm{t}, J=7.6 \mathrm{~Hz}, 1 \mathrm{H}), 3.74(\mathrm{~s}, 3 \mathrm{H}), 3.69$ (d, $J$ $=7.6 \mathrm{~Hz}, 2 \mathrm{H}), 3.38(\mathrm{br}, 1 \mathrm{H}) .{ }^{13} \mathrm{C}-\mathrm{NMR}\left(100 \mathrm{MHz}, \mathrm{CDCl}_{3}\right): 49.61,50.28,55.78$, $114.58,114.90,126.74,128.12,128.71,141.99,142.33,152.32$. IR (neat): 3390 , 3060, 3025, 2950, 2900, 2830, 1600, 1510, 1450, 1230, 1030, 820, 755, $700 \mathrm{~cm}^{-1}$. Exact mass ESI-MS $\mathrm{C}_{21} \mathrm{H}_{21} \mathrm{NOH}$ calculated: 304.1701 found: 304.1683 .

2.4. Synthesis of $\mathbf{3 c}$<smiles>Cc1ccc(NCC(c2ccccc2)c2ccccc2)cc1</smiles>

According to general procedure 1 (Method A), $1 \mathrm{a}$ (96 mg, $0.50 \mathrm{mmol}, 1.0$ equiv.), $\mathrm{B}\left(\mathrm{C}_{6} \mathrm{~F}_{5}\right)_{3}$ (12.4 mg, $0.023 \mathrm{mmol}, 0.05$ equiv.), 2c (( $p$-Toluidine) $64 \mathrm{mg}, 0.60 \mathrm{mmol}$, 1.2 equiv.) and $\mathrm{PhMe}_{2} \mathrm{SiH}$ ( $82 \mathrm{mg}, 0.60 \mathrm{mmol}, 1.2$ equiv.) were stirred in $\mathrm{PhCl}(4 \mathrm{~mL})$ for $3 \mathrm{~h}$ at $100{ }^{\circ} \mathrm{C}$. After flash column chromatography (PE:EtOAc $=96: 4 ; \mathrm{R}_{F}=0.10$ ) 3c was obtained as colorless oil (122 mg, $0.43 \mathrm{mmol}, 85 \%)$. ${ }^{1} \mathrm{H}-\mathrm{NMR}$ (400 $\mathrm{MHz}$, $\left.\mathrm{CDCl}_{3}\right): 7.34-7.21(\mathrm{~m}, 10 \mathrm{H}), 6.99(\mathrm{~d}, J=8.2 \mathrm{~Hz}, 2 \mathrm{H}), 6.53(\mathrm{~d}, J=8.4 \mathrm{~Hz}, 2 \mathrm{H}), 4.29$ $(\mathrm{t}, J=7.7 \mathrm{~Hz}, 1 \mathrm{H}), 3.73(\mathrm{~d}, J=7.7 \mathrm{~Hz}, 2 \mathrm{H}), 3.51(\mathrm{br}, 1 \mathrm{H}), 2.24(\mathrm{~s}, 3 \mathrm{H}) .{ }^{13} \mathrm{C}-\mathrm{NMR}$ $\left(100 \mathrm{MHz}, \mathrm{CDCl}_{3}\right): 145.52,142.33,129.75,128.69,128.13,126.90,126.72,113.36$, 50.23, 48.94, 20.37. IR (neat): 3400, 3025, 2915, 1615, 1520, 1490, 1320, 1255, $1130,1030,810,755,700,510 \mathrm{~cm}^{-1}$. Exact mass ESI-MS: $\mathrm{C}_{21} \mathrm{H}_{21} \mathrm{NH}$ calculated: 288.1752 found: 288.1728 .

2.5. Synthesis of $\mathbf{3 d}$<smiles>Clc1ccc(NCC(c2ccccc2)c2ccccc2)cc1</smiles>

According to general procedure 1 (Method A), $1 \mathrm{a}(96 \mathrm{mg}, 0.50 \mathrm{mmol}, 1.0$ equiv.), $\mathrm{B}\left(\mathrm{C}_{6} \mathrm{~F}_{5}\right)_{3}$ (12.5 mg, $0.024 \mathrm{mmol}, 0.05$ equiv.), 2d ((4-Chloroaniline) $77 \mathrm{mg}, 0.60$ mmol, 1.2 equiv.) and $\mathrm{PhMe}_{2} \mathrm{SiH}(81 \mathrm{mg}, 0.60 \mathrm{mmol}, 1.2$ equiv.) were stirred in $\mathrm{PhCl}$ $(4 \mathrm{~mL})$ for $3 \mathrm{~h}$ at $100{ }^{\circ} \mathrm{C}$. After flash column chromatography $\left(\mathrm{PE}: \mathrm{EtOAc}=95: 5 ; \mathrm{R}_{F}=\right.$ $0.23)$ 3d was obtained as yellowish oil (106 mg, $0.34 \mathrm{mmol}, 69 \%)$. ${ }^{1} \mathrm{H}-\mathrm{NMR}$ (400 $\left.\mathrm{MHz}, \mathrm{CDCl}_{3}\right): 7.34-7.22(\mathrm{~m}, 10 \mathrm{H}), 7.10(\mathrm{~d}, J=8.9 \mathrm{~Hz}, 2 \mathrm{H}), 6.50(\mathrm{~d}, J=8.9 \mathrm{~Hz}$, $2 \mathrm{H}), 4.27(\mathrm{t}, J=7.9 \mathrm{~Hz}, 1 \mathrm{H}), 3.70(\mathrm{~d}, J=7.9 \mathrm{~Hz}, 2 \mathrm{H}), 3.63(\mathrm{br}, 1 \mathrm{H}) .{ }^{13} \mathrm{C}-\mathrm{NMR}(100$ 
$\left.\mathrm{MHz}, \mathrm{CDCl}_{3}\right): 146.32,142.00,129.08,128.76,128.06,126.6,122.17,114.15,50.12$, 48.60. IR (neat): 3410, 3060, 3025, 2890, 1600, 1495, 1290, 1255, 1215, 1030, 1000 , 815, 755, 695, $505 \mathrm{~cm}^{-1}$. Exact mass ESI-MS: $\mathrm{C}_{20} \mathrm{H}_{18} \mathrm{NClH}$ calculated: 308.1206 found: 308.1260 .

2.6. Synthesis of $\mathbf{3 e}$<smiles>FC(F)(F)c1ccc(NCC(c2ccccc2)c2ccccc2)cc1</smiles>

According to general procedure 1 (Method A), $1 \mathrm{a}(98 \mathrm{mg}, 0.50 \mathrm{mmol}, 1.0$ equiv.), $\mathrm{B}\left(\mathrm{C}_{6} \mathrm{~F}_{5}\right)_{3}$ (12.8 mg, $0.025 \mathrm{mmol}, 0.05$ equiv.), 2e ((4-(Trifluoromethyl)aniline) $97 \mathrm{mg}$, $0.60 \mathrm{mmol}, 1.2$ equiv.) and $\mathrm{PhMe}_{2} \mathrm{SiH}(83 \mathrm{mg}, 0.60 \mathrm{mmol}, 1.2$ equiv.) were stirred in $\mathrm{PhCl}(4 \mathrm{~mL})$ for $3 \mathrm{~h}$ at $100{ }^{\circ} \mathrm{C}$. After flash column chromatography $(\mathrm{PE}: \mathrm{EtOAc}=$ 95:5; $\left.\mathrm{R}_{F}=0.33\right) 3 \mathrm{e}$ was obtained as colorless oil $(94 \mathrm{mg}, 0.28 \mathrm{mmol}, 55 \%) .{ }^{1} \mathrm{H}-\mathrm{NMR}$ $\left(400 \mathrm{MHz} \mathrm{CDCl}_{3}\right): 7.40(\mathrm{~d}, J=8.6 \mathrm{~Hz}, 2 \mathrm{H}), 7.37-7.31(\mathrm{~m}, 4 \mathrm{H}), 7.29-7.24(\mathrm{~m}, 6 \mathrm{H})$, $6.59(\mathrm{~d}, J=8.5 \mathrm{~Hz}, 2 \mathrm{H}), 4.29(\mathrm{t}, J=7.7 \mathrm{~Hz}, 1 \mathrm{H}), 3.96(\mathrm{br}, 1 \mathrm{H}), 3.77(\mathrm{~d}, J=7.7 \mathrm{~Hz}$, 2H). ${ }^{13} \mathrm{C}-\mathrm{NMR}\left(100 \mathrm{MHz}, \mathrm{CDCl}_{3}\right): 150.19,141.80,128.83,128.06,126.98,126.69$, 126.65, 126.61, 112.07, 50.12, 47.96. IR (neat): 3415, 3065, 3030, 2985, 2905, 1890 , $1615,1320,1105,1065,825,700 \mathrm{~cm}^{-1}$. Exact mass ESI-MS $\mathrm{C}_{21} \mathrm{H}_{18} \mathrm{NF}_{3} \mathrm{H}$ calculated: 342.1470 found: 342.1445 .

2.7. Synthesis of $\mathbf{3 f}$<smiles>CCOC(=O)c1cccc(NCC(c2ccccc2)c2ccccc2)c1</smiles>

According to general procedure 1 (Method A), 1a (95 mg, $0.50 \mathrm{mmol}, 1.0$ equiv.), $\mathrm{B}\left(\mathrm{C}_{6} \mathrm{~F}_{5}\right)_{3}$ (11.5 mg, $0.022 \mathrm{mmol}, 0.05$ equiv.), 2f ((Ethyl 3-aminobenzoate) $99 \mathrm{mg}$, $0.59 \mathrm{mmol}, 1.2$ equiv.) and $\mathrm{PhMe}_{2} \mathrm{SiH}(83 \mathrm{mg}, 0.61 \mathrm{mmol}, 1.2$ equiv.) were stirred in $\mathrm{PhCl}(4 \mathrm{~mL})$ for $3 \mathrm{~h}$ at $100{ }^{\circ} \mathrm{C}$. After flash column chromatography $(\mathrm{PE}:$ EtOAc $=$ 95:5; $\left.\mathrm{R}_{F}=0.13\right)$ 3f was obtained as colorless oil (118 mg, $\left.0.34 \mathrm{mmol}, 69 \%\right)$. $\mathrm{PhMe}_{2} \mathrm{SiOSiMe}_{2} \mathrm{Ph}$ was identified as impurity. ${ }^{1} \mathrm{H}-\mathrm{NMR}\left(400 \mathrm{MHz}, \mathrm{CDCl}_{3}\right): 7.39-$ $7.18(\mathrm{~m}, 13 \mathrm{H}), 6.74(\mathrm{dd}, J=8.1 \mathrm{~Hz}, J=2.4 \mathrm{~Hz}, 1 \mathrm{H}), 4.34(\mathrm{q}, J=7.1 \mathrm{~Hz}, 2 \mathrm{H}), 4.29(\mathrm{t}$, $J=7.5 \mathrm{~Hz}, 1 \mathrm{H}), 3.77(\mathrm{~d} J=7.1 \mathrm{~Hz}, 2 \mathrm{H}), 3.76(\mathrm{br}, 1 \mathrm{H}), 1.37(\mathrm{t}, J=7.1 \mathrm{~Hz}, 3 \mathrm{H}) .{ }^{13} \mathrm{C}-$ NMR $\left(100 \mathrm{MHz}, \mathrm{CDCl}_{3}\right)$ : 166.95, 147.76, 133.00, 131.44, 129.13, 128.77, 128.09, 126.86, 118.71, 117.33, 113.57, 60.83, 50.26, 48.38, 14.33. IR (neat): 3340, 3060, 3030, 2900, 1710, 1605, 1490, 1430, 1330, 1275, 1240, 1105, 1025, 990, 870, 780, $750,700,685,545,470 \mathrm{~cm}^{-1}$. Exact mass ESI-MS $\mathrm{C}_{23} \mathrm{H}_{23} \mathrm{NO}_{2} \mathrm{H}$ calculated: 346.1807 found: 346.1764 . 
2.8. Synthesis of $\mathbf{3 g}$<smiles>Cc1cccc(C)c1NCC(c1ccccc1)c1ccccc1</smiles>

$3 g$

According to general procedure 1 (Method A), $1 \mathbf{a}(98 \mathrm{mg}, 0.50 \mathrm{mmol}, 1.0$ equiv.), $\mathrm{B}\left(\mathrm{C}_{6} \mathrm{~F}_{5}\right)_{3}$ (12.8 mg, $0.025 \mathrm{mmol}, 0.05$ equiv.), $2 \mathrm{~g}$ ((2,6-dimethylaniline) $73 \mathrm{mg}, 0.60$ mmol, 1.2 equiv.) and $\mathrm{PhMe}_{2} \mathrm{SiH}(82 \mathrm{mg}, 0.60 \mathrm{mmol}, 1.2$ equiv.) were stirred in $\mathrm{PhCl}$ $(4 \mathrm{~mL})$ for $3 \mathrm{~h}$ at $100{ }^{\circ} \mathrm{C}$. After flash column chromatography (PE:EtOAc $=97: 3 ; \mathrm{R}_{F}=$ $0.17) 3 \mathrm{~g}$ was obtained as colorless oil (127 mg, $0.42 \mathrm{mmol}, 84 \%)$. ${ }^{1} \mathrm{H}-\mathrm{NMR}$ (400 $\left.\mathrm{MHz}, \mathrm{CDCl}_{3}\right): 7.35-7.21(\mathrm{~m}, 10 \mathrm{H}), 7.03(\mathrm{~d}, J=7.5 \mathrm{~Hz}, 2 \mathrm{H}), 6.88(\mathrm{t}, J=7.5 \mathrm{~Hz}, 1 \mathrm{H})$, $4.30(\mathrm{t}, J=7.7 \mathrm{~Hz}, 1 \mathrm{H}), 3.73(\mathrm{~d}, J=7.7 \mathrm{~Hz}, 2 \mathrm{H}), 3.22(\mathrm{br}, 1 \mathrm{H}), 2.11(\mathrm{~s}, 6 \mathrm{H}) .{ }^{13} \mathrm{C}-\mathrm{NMR}$ $\left(\mathrm{CDCl}_{3}\right): 145.68,142.51,128.94,128.82,128.74,128.12,126.77,121.60,52.75$, 51.84, 18.31. IR (neat): 3415, 3060, 3030, 2985, 1735, 1615, 1320, 1105, 1065, 825, $700 \mathrm{~cm}^{-1}$. Exact mass ESI-MS C ${ }_{22} \mathrm{H}_{23} \mathrm{NH}$ calculated: 302.1909 found: 302.1920 .

\subsection{Synthesis of $\mathbf{3 h}$}<smiles>Cc1cc(Br)cc(C)c1NCC(c1ccccc1)c1ccccc1</smiles>

According to general procedure 1 (Method A), $1 \mathrm{a}(96 \mathrm{mg}, 0.50 \mathrm{mmol}, 1.0$ equiv.), $\mathrm{B}\left(\mathrm{C}_{6} \mathrm{~F}_{5}\right)_{3}$ (12.5 mg, $0.024 \mathrm{mmol}, 0.05$ equiv.), 2h ((4-Bromo-2,6-dimethylaniline) 120 $\mathrm{mg}, 0.60 \mathrm{mmol}, 1.2$ equiv.) and $\mathrm{PhMe}_{2} \mathrm{SiH}(81 \mathrm{mg}, 0.60 \mathrm{mmol}, 1.2$ equiv.) were stirred in $\mathrm{PhCl}(4 \mathrm{~mL})$ for $3 \mathrm{~h}$ at $100{ }^{\circ} \mathrm{C}$. After flash column chromatography (PE:EtOAc $\left.=95: 5 ; \mathrm{R}_{F}=0.21\right) \mathbf{3 h}$ was obtained as yellowish oil $(110 \mathrm{mg}, 0.29 \mathrm{mmol}$, 58\%). ${ }^{1} \mathrm{H}-\mathrm{NMR}\left(400 \mathrm{MHz}, \mathrm{CDCl}_{3}\right): 7.34-7.21(\mathrm{~m}, 10 \mathrm{H}), 7.05(\mathrm{~s}, 2 \mathrm{H}), 4.16(\mathrm{t}, J=$ $8.3,1 \mathrm{H}), 3.59(\mathrm{~d}, J=7.9,2 \mathrm{H}), 3.08(\mathrm{br}, 1 \mathrm{H}), 1.96,(\mathrm{~s}, 6 \mathrm{H}) \cdot{ }^{13} \mathrm{C}-\mathrm{NMR}(100 \mathrm{MHz}$, $\mathrm{CDCl}_{3}$ ): 144.76, 142.21, 131.22, 130.96, 128.78, 128.04, 126.85, 113.73, 52.60, 51.73, 18.14. IR (neat): 3395, 3025, 3060, 2920, 1660, 1600, 1470, 1280, 1220, 1030, $920,860,700,580,540 \mathrm{~cm}^{-1}$. Exact mass ESI-MS $\mathrm{C}_{22} \mathrm{H}_{22} \mathrm{BrNH}$ calculated: 380.1014 found: 380.0930 . 
2.10. Synthesis of $\mathbf{3 i}$<smiles>c1ccc(C(CNc2ccc3ccccc3c2)c2ccccc2)cc1</smiles>

According to general procedure 1 (Method A), 1a (98 mg, $0.50 \mathrm{mmol}, 1.0$ equiv.), $\mathrm{B}\left(\mathrm{C}_{6} \mathrm{~F}_{5}\right)_{3}$ (12.8 mg, $0.025 \mathrm{mmol}, 0.05$ equiv.), 2i ((2-Naphthylamine) $86 \mathrm{mg}, 0.60$ mmol, 1.2 equiv.) and $\mathrm{PhMe}_{2} \mathrm{SiH}$ (82 $\mathrm{mg}, 0.60 \mathrm{mmol}, 1.2$ equiv.) were stirred in $\mathrm{PhCl}$ $(4 \mathrm{~mL})$ for $3 \mathrm{~h}$ at $100{ }^{\circ} \mathrm{C}$. After flash column chromatography (PE:EtOAc $=95: 5 ; \mathrm{R}_{F}=$ $0.23) 3 \mathbf{i}$ was obtained as orange oil $(96 \mathrm{mg}, 0.30 \mathrm{mmol}, 60 \%) .{ }^{1} \mathrm{H}-\mathrm{NMR}(400 \mathrm{MHz}$, $\left.\mathrm{CDCl}_{3}\right): 7.66(\mathrm{~d}, J=8.0 \mathrm{~Hz}, 1 \mathrm{H}), 7.61(\mathrm{~d}, J=8.3 \mathrm{~Hz}, 1 \mathrm{H}), 7.60(\mathrm{~d}, J=8.8 \mathrm{~Hz}, 1 \mathrm{H})$, $7.39-7.24(\mathrm{~m}, 11 \mathrm{H}), 7.20(\mathrm{t}, J=7.5 \mathrm{~Hz}, 1 \mathrm{H}), 6.87(\mathrm{~s}, 1 \mathrm{H}), 6.79(\mathrm{dd}, J=8.7 \mathrm{~Hz}, J=$ $2.2 \mathrm{~Hz}, 1 \mathrm{H}), 4.38(\mathrm{t}, J=7.6 \mathrm{~Hz}, 1 \mathrm{H}), 3.86(\mathrm{~d}, J=7.6 \mathrm{~Hz}, 2 \mathrm{H}) .{ }^{13} \mathrm{C}-\mathrm{NMR}(100 \mathrm{MHz}$, $\left.\mathrm{CDCl}_{3}\right): 145.40,142.21,135.16,128.94,128.77,128.66,128.60,128.16,127.62$, $126.85,126.35,125.94,122.10,118.11,104.83,50.06,48.52$. IR (neat): 3405, 3060, $3025,2980,2900,1630,1600,1520,830,700 \mathrm{~cm}^{-1}$. Exact mass ESI-MS $\mathrm{C}_{24} \mathrm{H}_{21} \mathrm{NH}$ calculated: 324.1752 found: 324.1743 .

2.11. Synthesis of $\mathbf{3 j}$<smiles>c1ccc(C(CNc2cccc3ccccc23)c2ccccc2)cc1</smiles>

3j

According to general procedure 1 (Method A), $1 \mathrm{a}(98 \mathrm{mg}, 0.50 \mathrm{mmol}, 1.0$ equiv), $\mathrm{B}\left(\mathrm{C}_{6} \mathrm{~F}_{5}\right)_{3}$ (12.8 mg, $0.025 \mathrm{mmol}, 0.05$ equiv.), 2j ((1-Naphthylamine) $86 \mathrm{mg}, 0.60$ mmol, 1.2 equiv.) and $\mathrm{PhMe}_{2} \mathrm{SiH}$ ( $82 \mathrm{mg}, 0.60 \mathrm{mmol}, 1.2$ equiv) were stirred in $\mathrm{PhCl}$ $(4 \mathrm{~mL})$ for $3 \mathrm{~h}$ at $100{ }^{\circ} \mathrm{C}$. After flash column chromatography (PE:EtOAc $=95: 5 ; \mathrm{R}_{F}=$ $0.33)$ 3j was obtained as yellowish oil ( $84 \mathrm{mg}, 0.26 \mathrm{mmol}, 52 \%) . \mathrm{PhMe}_{2} \mathrm{SiOSiMe}_{2} \mathrm{Ph}$ was identified as impurity. ${ }^{1} \mathrm{H}-\mathrm{NMR}\left(400 \mathrm{MHz}, \mathrm{CDCl}_{3}\right): 7.77(\mathrm{~d}, J=8.3 \mathrm{~Hz}, 1 \mathrm{H})$, 7.49-7.27 (m, 15H), $6.72(\mathrm{~d}, J=7.5 \mathrm{~Hz}, 1 \mathrm{H}), 4.49(\mathrm{t}, J=7.6 \mathrm{~Hz}, 1 \mathrm{H}), 4.35(\mathrm{br}, 1 \mathrm{H})$, $3.91(\mathrm{~d}, J=7.6 \mathrm{~Hz}, 2 \mathrm{H}) .{ }^{13} \mathrm{C}-\mathrm{NMR}\left(100 \mathrm{MHz}, \mathrm{CDCl}_{3}\right): 142.09,134.28,128.82$, $128.61,128.18,126.94,126.49,125.76,124.88,123.61,119.67,110.00,50.01,49.09$. IR (neat): 3420, 3060, 3025, 2955, 2895, 1950, 1810, 1730, 1580, 1525, 1475, 1410, $770,700 \mathrm{~cm}^{-1}$. Exact mass ESI-MS $\mathrm{C}_{24} \mathrm{H}_{21} \mathrm{NH}$ calculated: 324.1752 found: 324.1701 .

2.12. Unsuccessful synthesis of $\mathbf{3 k}, \mathbf{3 1}$, and $\mathbf{3 m}$

According to general procedure 1 (Method A), $1 \mathbf{a}\left(0.50\right.$ mmol, 1.0 equiv.), $\mathrm{B}\left(\mathrm{C}_{6} \mathrm{~F}_{5}\right)_{3}$ (0.023 mmol, 0.05 equiv.), $2 \mathbf{k}\left(0.60 \mathrm{mmol}, 1.2\right.$ equiv.) and $\mathrm{PhMe}_{2} \mathrm{SiH}(0.60 \mathrm{mmol}$, 
1.2 equiv.) were stirred in $\mathrm{PhCl}(4 \mathrm{~mL})$ for $3 \mathrm{~h}$ at $100{ }^{\circ} \mathrm{C}$. NMR analysis of the crude reaction mixture indicated no formation of $\mathbf{3 k}$.

According to general procedure 1 (Method A), $1 \mathrm{a}\left(0.50 \mathrm{mmol}, 1.0\right.$ equiv.), $\mathrm{B}\left(\mathrm{C}_{6} \mathrm{~F}_{5}\right)_{3}$ (0.023 mmol, 0.05 equiv.), $2 \mathrm{l}$ (0.60 mmol, 1.2 equiv.) and $\mathrm{PhMe}_{2} \mathrm{SiH}(0.60 \mathrm{mmol}, 1.2$ equiv.) were stirred in $\mathrm{PhCl}(4 \mathrm{~mL})$ for $3 \mathrm{~h}$ at $100{ }^{\circ} \mathrm{C}$. NMR analysis of the crude reaction mixture indicated no formation of 31 .

According to general procedure 1 (Method A), $1 \mathrm{a}\left(0.50 \mathrm{mmol}, 1.0\right.$ equiv.), $\mathrm{B}\left(\mathrm{C}_{6} \mathrm{~F}_{5}\right)_{3}$ (0.023 mmol, 0.05 equiv.), $2 \mathrm{~m}\left(0.60 \mathrm{mmol}, 1.2\right.$ equiv.) and $\mathrm{PhMe}_{2} \mathrm{SiH}(0.60 \mathrm{mmol}$, 1.2 equiv.) were stirred in $\mathrm{PhCl}(4 \mathrm{~mL})$ for $3 \mathrm{~h}$ at $100{ }^{\circ} \mathrm{C}$. NMR analysis of the crude reaction mixture indicated no formation of $\mathbf{3 m}$.

2.13. Synthesis of $\mathbf{3 n}$<smiles>CC(C)(C)c1ccc(C(CNc2ccccc2)c2ccccc2)cc1</smiles>

According to general procedure 1 (Method A), $1 \mathbf{b}(126 \mathrm{mg}, 0.50 \mathrm{mmol}, 1.0$ equiv), $\mathrm{B}\left(\mathrm{C}_{6} \mathrm{~F}_{5}\right)_{3}(12.5 \mathrm{mg}, 0.024 \mathrm{mmol}, 0.05$ equiv.), $2 \mathrm{a}(56 \mathrm{mg}, 0.60 \mathrm{mmol}, 1.2$ equiv.) and $\mathrm{PhMe}_{2} \mathrm{SiH}$ (81 mg, $0.60 \mathrm{mmol}, 1.2$ equiv.) were stirred in $\mathrm{PhCl}(4 \mathrm{~mL})$ for $3 \mathrm{~h}$ at 100 ${ }^{\circ} \mathrm{C}$. After flash column chromatography (PE:EtOAc $=95: 5 ; \mathrm{R}_{F}=0.28$ ) 3n was obtained as colorless oil ( $164 \mathrm{mg}, 0.50 \mathrm{mmol},>99 \%)$. ${ }^{1} \mathrm{H}-\mathrm{NMR}\left(400 \mathrm{MHz}, \mathrm{CDCl}_{3}\right.$ ): $7.34-7.14(\mathrm{~m}, 11 \mathrm{H}), 6.71(\mathrm{t}, J=6.8 \mathrm{~Hz}, 1 \mathrm{H}), 6.60(\mathrm{~d}, J=8.5 \mathrm{~Hz}, 2 \mathrm{H}), 4.26(\mathrm{t}, J=$ $7.7 \mathrm{~Hz}, 1 \mathrm{H}), 3.72(\mathrm{~d}, J=7.9 \mathrm{~Hz}, 2 \mathrm{H}), 3.04(\mathrm{br}, 1 \mathrm{H}), 1.30(\mathrm{~s}, 9 \mathrm{H}) .{ }^{13} \mathrm{C}-\mathrm{NMR}(100$ $\mathrm{MHz}, \mathrm{CDCl}_{3}$ ): 149.51, 147.85, 142.44, 139.12, 129.24, 128.67, 128.15, 127.66, $126.70,125.58,117.55,113.11,49.84,48.57,34.40,31.33$. IR (neat): 3055, 3025, 2960, 2870, 1600, 1505, 1475, 1320, 1260, 1110, 835, 745, 690, $505 \mathrm{~cm}^{-1}$. Exact mass ESI-MS $\mathrm{C}_{24} \mathrm{H}_{27} \mathrm{NH}$ calculated: 330.2222 found: 330.2209 .

2.14. Synthesis of $\mathbf{3 o}$<smiles>Brc1ccc(C(CNc2ccccc2)c2ccccc2)cc1</smiles>

According to general procedure 1 (Method A), 1c (138 mg, $0.50 \mathrm{mmol}, 1.0$ equiv.), $\mathrm{B}\left(\mathrm{C}_{6} \mathrm{~F}_{5}\right)_{3}(12.5 \mathrm{mg}, 0.024 \mathrm{mmol}, 0.05$ equiv.), $2 \mathrm{a}(56 \mathrm{mg}, 0.60 \mathrm{mmol}, 1.2$ equiv.) and $\mathrm{PhMe}_{2} \mathrm{SiH}$ (81 mg, $0.60 \mathrm{mmol}, 1.2$ equiv.) were stirred in $\mathrm{PhCl}(4 \mathrm{~mL})$ for $3 \mathrm{~h}$ at 100 ${ }^{\circ} \mathrm{C}$. After flash column chromatography (PE:EtOAc $=95: 5 ; \mathrm{R}_{F}=0.17$ ) 3o was obtained as colorless oil ( $156 \mathrm{mg}, 0.44 \mathrm{mmol}, 88 \%)$. ${ }^{1} \mathrm{H}-\mathrm{NMR}\left(400 \mathrm{MHz}, \mathrm{CDCl}_{3}\right.$ ): $7.44(\mathrm{~d}, J=8.3 \mathrm{~Hz}, 2 \mathrm{H}), 7.35-7.12(\mathrm{~m}, 9 \mathrm{H}), 6.72(\mathrm{t}, J=7.6 \mathrm{~Hz}, 1 \mathrm{H}), 6.59(\mathrm{~d}, J=8.1$ 
$\mathrm{Hz}, 2 \mathrm{H}), 4.25(\mathrm{t}, J=7.4 \mathrm{~Hz}, 1 \mathrm{H}), 3.71(\mathrm{~d}, J=8.5 \mathrm{~Hz}, 2 \mathrm{H}), 3.60(\mathrm{br}, 1 \mathrm{H}) .{ }^{13} \mathrm{C}-\mathrm{NMR}$ $\left(100 \mathrm{MHz}, \mathrm{CDCl}_{3}\right): 147.55,141.62,141.32,131.78,129.85,129.31,128.83,128.02$, $127.00,120.64,117.80,113.11,49.63,48.35$. IR (neat): 3405, 3050, 3025, 1600, $1505,1490,1450,1430,1315,1255,1180,1070,1010,820,745,715,690,540,505$ $\mathrm{cm}^{-1}$. Exact mass ESI-MS $\mathrm{C}_{20} \mathrm{H}_{18} \mathrm{BrNH}$ calculated: 352.0701 found: 352.0701 .

2.15. Synthesis of $\mathbf{3 p}$<smiles>Clc1ccc(C(CNc2ccccc2)c2ccccc2)cc1</smiles>

According to general procedure 1 (Method A), 1 d (115 mg, $0.50 \mathrm{mmol}, 1.0$ equiv.), $\mathrm{B}\left(\mathrm{C}_{6} \mathrm{~F}_{5}\right)_{3}(11.5 \mathrm{mg}, 0.022 \mathrm{mmol}, 0.05$ equiv.), $2 \mathrm{a}(56 \mathrm{mg}, 0.60 \mathrm{mmol}, 1.2$ equiv.) and $\mathrm{PhMe}_{2} \mathrm{SiH}$ (81 mg, $0.60 \mathrm{mmol}, 1.2$ equiv.) were stirred in $\mathrm{PhCl}(4 \mathrm{~mL})$ for $3 \mathrm{~h}$ at 100 ${ }^{\circ} \mathrm{C}$. After flash column chromatography (PE:EtOAc $=92: 8 ; \mathrm{R}_{F}=0.28$ ) $\mathbf{3 p}$ was obtained as colorless oil (133 mg, $0.43 \mathrm{mmol}, 87 \%)$. ${ }^{1} \mathrm{H}-\mathrm{NMR}\left(400 \mathrm{MHz}, \mathrm{CDCl}_{3}\right)$ : $7.35-7.17(\mathrm{~m}, 11 \mathrm{H}), 6.72(\mathrm{t}, J=7.3 \mathrm{~Hz}, 1 \mathrm{H}), 6.59(\mathrm{~d}, J=8.6 \mathrm{~Hz}, 2 \mathrm{H}), 4.27(\mathrm{t}, J=$ $7.6 \mathrm{~Hz}, 1 \mathrm{H}), 3.71(\mathrm{~d}, J=7.7 \mathrm{~Hz}, 2 \mathrm{H}), 3.59$ (br, $1 \mathrm{H}) .{ }^{13} \mathrm{C}-\mathrm{NMR}\left(100 \mathrm{MHz}, \mathrm{CDCl}_{3}\right)$ : $147.56,141.71,140.78,132.56,129.46,129.30,128.83,128.02,126.98,117.80$, 113.11, 49.57, 48.41. IR (neat): 3410, 3050, 2025, 1600, 1490, 1504, 1450, 1315, $1255,1090,1015,820,745,690,540,505,490 \mathrm{~cm}^{-1}$. Exact mass ESI-MS $\mathrm{C}_{20} \mathrm{H}_{18} \mathrm{ClNH}$ calculated: 308.1206 found: 308.1172 .

2.16. Synthesis of $\mathbf{3 q}$<smiles>O=[N+]([O-])c1ccc(C(CNc2ccccc2)c2ccccc2)cc1</smiles>

According to general procedure 1 (Method A), 1e (120 mg, $0.50 \mathrm{mmol}, 1.0$ equiv.), $\mathrm{B}\left(\mathrm{C}_{6} \mathrm{~F}_{5}\right)_{3}(12.5 \mathrm{mg}, 0.024 \mathrm{mmol}, 0.05$ equiv.), $2 \mathrm{a}(56 \mathrm{mg}, 0.60 \mathrm{mmol}, 1.2$ equiv.) and $\mathrm{PhMe}_{2} \mathrm{SiH}$ (82 mg, $0.60 \mathrm{mmol}, 1.2$ equiv.) were stirred in $\mathrm{PhCl}(4 \mathrm{~mL})$ for $3 \mathrm{~h}$ at 100 ${ }^{\circ} \mathrm{C}$. After flash column chromatography (PE:EtOAc $=95: 5 ; \mathrm{R}_{F}=0.07$ ) $\mathbf{3 q}$ was obtained as orange oil (65 mg, $0.20 \mathrm{mmol}, 40 \%)$. $\mathrm{PhMe}_{2} \mathrm{SiOSiMe}_{2} \mathrm{Ph}$ and 1e were identified as impurities. ${ }^{1} \mathrm{H}-\mathrm{NMR}\left(400 \mathrm{MHz}, \mathrm{CDCl}_{3}\right): 8.17(\mathrm{~d}, J=8.8 \mathrm{~Hz}, 2 \mathrm{H}), 7.43$ $(\mathrm{d}, J=8.8 \mathrm{~Hz}, 2 \mathrm{H}), 7.40-7.16(\mathrm{~m}, 7 \mathrm{H}), 6.75(\mathrm{t}, J=7.7 \mathrm{~Hz}, 1 \mathrm{H}), 6.59(\mathrm{~d}, J=8.3 \mathrm{~Hz}$, $2 \mathrm{H}), \mathrm{m}(\mathrm{t}, J=7.7 \mathrm{~Hz}, 1 \mathrm{H}), 4.42-4.38(\mathrm{~m}, 1 \mathrm{H}), 3.81-3.76(\mathrm{~m}, 2 \mathrm{H}) .{ }^{13} \mathrm{C}-\mathrm{NMR}(100$ $\left.\mathrm{MHz}, \mathrm{CDCl}_{3}\right): 150.02,147.32,129.39,129.09,128.98,128.08,127.58,127.44$, 126.59, 123.91, 123.06, 118.09, 113.13, 50.06, 48.21. IR (neat): 3405, 3055, 3025, 2850, 1930, 1690, 1600, 1515, 1450, 1430, 1340, 1320, 1215, 1155, 1110, 1075, 1030, 850, 745, 695, 545, 510, $470 \mathrm{~cm}^{-1}$. Exact mass ESI-MS $\mathrm{C}_{20} \mathrm{H}_{18} \mathrm{~N}_{2} \mathrm{O}_{2} \mathrm{H}$ calculated: 319.1447 found: 319.1390 . 
<smiles>c1ccc(NCC(c2ccccc2)c2ccccc2)cc1</smiles>

$3 a$

According to general procedure 1 (Method B), 1 f (98 mg, $0.50 \mathrm{mmol}, 1.0$ equiv.), $\mathrm{B}\left(\mathrm{C}_{6} \mathrm{~F}_{5}\right)_{3}$ (25.6 mg, $0.05 \mathrm{mmol}, 0.10$ equiv.), $2 \mathrm{a}$ (56 mg, $0.60 \mathrm{mmol}, 1.2$ equiv.) and $\mathrm{PhMe}_{2} \mathrm{SiH}$ (82 mg, $0.60 \mathrm{mmol}, 1.2$ equiv.) were stirred in 1,4-dioxane $(0.5 \mathrm{~mL})$ and $\mathrm{PhCl}(3.5 \mathrm{~mL})$ for $3 \mathrm{~h}$ at $100{ }^{\circ} \mathrm{C}$. According to $\mathrm{NMR}$ analysis of the crude product (internal standard: 1,3,5-trimethoxybenzene) 3a was obtained (87 $\mathrm{mg}, 0.32 \mathrm{mmol}$, 64\%). NMR data matches with those reported at $\mathbf{2 . 2}$ and in literature. ${ }^{[\mathrm{S} 1]}$

2.18. Synthesis of $\mathbf{3 r}$<smiles>Brc1ccccc1CCNc1ccccc1</smiles>

According to general procedure 1 (Method B), $1 \mathrm{~g}$ (60 mg, $0.50 \mathrm{mmol}, 1.0$ equiv.), $\mathrm{B}\left(\mathrm{C}_{6} \mathrm{~F}_{5}\right)_{3}$ (25.6 mg, $0.05 \mathrm{mmol}, 0.10$ equiv.), 2a (56 mg, $0.60 \mathrm{mmol}, 1.2$ equiv.) and $\mathrm{PhMe}_{2} \mathrm{SiH}$ (82 mg, $0.60 \mathrm{mmol}, 1.2$ equiv.) were stirred in 1,4-dioxane $(0.5 \mathrm{~mL})$ and $\mathrm{PhCl}(3.5 \mathrm{~mL})$ for $3 \mathrm{~h}$ at $100{ }^{\circ} \mathrm{C}$. According to $\mathrm{NMR}$ analysis of the crude product (internal standard: 1,3,5-trimethoxybenzene) $3 \mathbf{r}$ was obtained $(30 \mathrm{mg}, 0.15 \mathrm{mmol}$, $30 \%) .{ }^{1} \mathrm{H}-\mathrm{NMR}\left(400 \mathrm{MHz}, \mathrm{CDCl}_{3}\right): 3.42(\mathrm{t}, J=7.0 \mathrm{~Hz}, 2 \mathrm{H}), 2.93(\mathrm{t}, J=7.0 \mathrm{~Hz}, 2 \mathrm{H})$. (Aromatic signals overlap with those of other compounds). Exact mass ESI-MS $\mathrm{C}_{14} \mathrm{H}_{15} \mathrm{NH}$ calculated: 198.1283 found: 198.1264 . NMR data matches with those reported in literature. ${ }^{[\mathrm{S} 2]}$

2.19. Synthesis of $3 \mathrm{~s}$<smiles>Clc1ccc(CCNc2ccccc2)cc1</smiles>

According to general procedure 1 (Method B), $\mathbf{1 h}$ (77 $\mathrm{mg}, 0.50 \mathrm{mmol}, 1.0$ equiv.), $\mathrm{B}\left(\mathrm{C}_{6} \mathrm{~F}_{5}\right)_{3}$ (25.6 mg, $0.05 \mathrm{mmol}, 0.10$ equiv.), $2 \mathrm{a}(56 \mathrm{mg}, 0.60 \mathrm{mmol}, 1.2$ equiv.) and $\mathrm{PhMe}_{2} \mathrm{SiH}$ (82 mg, $0.60 \mathrm{mmol}, 1.2$ equiv.) were stirred in 1,4-dioxane $(0.5 \mathrm{~mL})$ and $\mathrm{PhCl}(3.5 \mathrm{~mL})$ for $3 \mathrm{~h}$ at $100{ }^{\circ} \mathrm{C}$. According to NMR analysis of the crude product (internal standard: 1,3,5-trimethoxybenzene) $3 \mathrm{~s}$ was obtained $(30 \mathrm{mg}, 0.13 \mathrm{mmol}$, 26\%). ${ }^{1} \mathrm{H}-\mathrm{NMR}\left(400 \mathrm{MHz}, \mathrm{CDCl}_{3}\right): 3.38(\mathrm{t}, J=6.9 \mathrm{~Hz}, 2 \mathrm{H}), 2.89(\mathrm{t}, J=7.0 \mathrm{~Hz}, 2 \mathrm{H})$. (Aromatic signals overlap with those of other compounds) Exact mass ESI-MS $\mathrm{C}_{14} \mathrm{H}_{14} \mathrm{ClNH}$ calculated: 232.0893 found: 232.0867 . NMR data matches with those reported in literature. ${ }^{[\mathrm{S} 3]}$ 
<smiles>CC(CNc1ccccc1)c1ccc(C(C)(C)C)cc1</smiles>

According to general procedure 1 (Method B), 1i (95 mg, $0.50 \mathrm{mmol}, 1.0$ equiv.), $\mathrm{B}\left(\mathrm{C}_{6} \mathrm{~F}_{5}\right)_{3}(25.6 \mathrm{mg}, 0.05 \mathrm{mmol}, 0.10$ equiv.), $2 \mathrm{a}$ (56 mg, $0.60 \mathrm{mmol}, 1.2$ equiv.) and $\mathrm{PhMe}_{2} \mathrm{SiH}$ (81 mg, $0.60 \mathrm{mmol}, 1.2$ equiv.) were stirred in 1,4-dioxane $(0.5 \mathrm{~mL})$ and $\mathrm{PhCl}(3.5 \mathrm{~mL})$ for $3 \mathrm{~h}$ at $100{ }^{\circ} \mathrm{C}$. According to $\mathrm{NMR}$ analysis of the crude product (internal standard: mesitylene) $3 \mathrm{t}$ was obtained (32 mg, $0.12 \mathrm{mmol}, 24 \%$ ). ${ }^{1} \mathrm{H}-\mathrm{NMR}$ $\left(400 \mathrm{MHz}, \mathrm{CDCl}_{3}\right): 6.76(\mathrm{tt}, J=7.4 \mathrm{~Hz}, J=1.1 \mathrm{~Hz}, 1 \mathrm{H}), 6.72-6.67(\mathrm{~m}, 2 \mathrm{H}), 3.32$ $(\mathrm{dd}, J=12.3 \mathrm{~Hz}, J=6.3 \mathrm{~Hz}, 1 \mathrm{H}), 3.23(\mathrm{dd}, J=12.2 \mathrm{~Hz}, J=8.0 \mathrm{~Hz}, 1 \mathrm{H}), 3.32(\mathrm{dq}, J=$ $13.8 \mathrm{~Hz}, J=6.7 \mathrm{~Hz}, 1 \mathrm{H}), 1.38(\mathrm{~s}, 9 \mathrm{H}), 1.25(\mathrm{~d}, J=6.0 \mathrm{~Hz}, 3 \mathrm{H})$. (Some aromatic signals overlap with those of other compounds) NMR data matches with those reported in literature. ${ }^{[\mathrm{S} 4]}$

\subsection{Unsuccessful synthesis of $\mathbf{3 u}$}

According to general procedure 1 (Method B), 1j (64 mg, $0.50 \mathrm{mmol}, 1.0$ equiv.), $\mathrm{B}\left(\mathrm{C}_{6} \mathrm{~F}_{5}\right)_{3}(25.6 \mathrm{mg}, 0.05 \mathrm{mmol}, 0.10$ equiv.), $2 \mathrm{a}(56 \mathrm{mg}, 0.60 \mathrm{mmol}, 1.2$ equiv.) and $\mathrm{PhMe}_{2} \mathrm{SiH}$ (82 $\mathrm{mg}, 0.60 \mathrm{mmol}, 1.2$ equiv.) were stirred in 1,4-dioxane $(0.5 \mathrm{~mL})$ and $\mathrm{PhCl}(4 \mathrm{~mL})$ for $3 \mathrm{~h}$ at $100{ }^{\circ} \mathrm{C}$. NMR analysis of the crude reaction mixture indicated no formation of $\mathbf{3 u}$.

\subsection{Synthesis of $\mathbf{1 b}$}

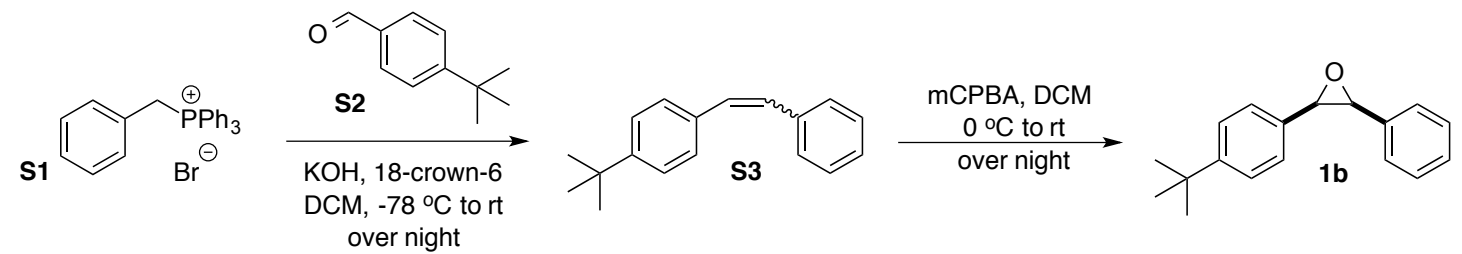

According to general procedure 2, S2 $(1.78 \mathrm{~g}, 11.0 \mathrm{mmol})$ was transformed into $\mathbf{S 3}$. After purification of the crude product via flash column chromatography $\left(\mathrm{PE}_{\mathrm{Et}} \mathrm{E}=\right.$ 90:10, $\left.\mathrm{R}_{F}=0.70\right) \mathbf{S 3}$ was obtained as a colorless oil $(1.82 \mathrm{~g}, 7.69 \mathrm{mmol}, 77 \%, E: Z=$ $32: 68)$ that crystallized upon storing between $5-7{ }^{\circ} \mathrm{C}$. NMR data matches with those reported in literature $\left(\mathrm{E}^{[\mathrm{S5}]}\right.$ and $\left.\mathrm{Z}^{[\mathrm{S6}]}\right)$.

According to general procedure $2, \mathbf{S 3}(1.80 \mathrm{~g}, 7.59 \mathrm{mmol})$ was stirred with $m \mathrm{CPBA}$ (70-75\% in water, $2.56 \mathrm{~g}$ ) in $\mathrm{CH}_{2} \mathrm{Cl}_{2}(40 \mathrm{~mL})$. After purification of the crude product via flash column chromatography $\left(\mathrm{PE}: \mathrm{Et}_{2} \mathrm{O}=95: 5, \mathrm{R}_{F}=0.40\right.$ in $\left.\mathrm{PE}: \mathrm{Et}_{2} \mathrm{O}=90: 10\right) \mathbf{1 b}$ was obtained as a colorless oil (998 $\mathrm{mg}, 3.96 \mathrm{mmol}, 52 \%)$ that crystallized upon storing between $5-7{ }^{\circ} \mathrm{C}$. The overall yield is $1.58 \mathrm{~g}, 6.29 \mathrm{mmol}, 83 \%$ and corresponds to a cis:trans ratio of $63: 37$ with $\mathbf{1 b}$-trans $\left(586 \mathrm{mg}, 2.33 \mathrm{mmol}, 31 \%, \mathrm{R}_{F}\right.$ $=0.49$ in PE: $\mathrm{Et}_{2} \mathrm{O}=90: 10$ ??). ${ }^{1} \mathrm{H}-\mathrm{NMR}\left(400 \mathrm{MHz}, \mathrm{CDCl}_{3}\right): 7.22-7.15(\mathrm{~m}, 7 \mathrm{H}), 7.08$ $(\mathrm{d}, J=8.4 \mathrm{~Hz}, 2 \mathrm{H}), 4.33(\mathrm{~d}, J=4.3 \mathrm{~Hz}, 1 \mathrm{H}), 4.31(\mathrm{~d}, J=4.3 \mathrm{~Hz}, 1 \mathrm{H}), 1.22(\mathrm{~s}, 9 \mathrm{H})$. ${ }^{13} \mathrm{C}-\mathrm{NMR}\left(100 \mathrm{MHz}, \mathrm{CDCl}_{3}\right): 150.42,134.56,131.24,127.76,127.42,126.93$, 
126.66, 124.71, 59.89, 34.44, 31.23. IR (neat): 2955, 2905, 2870, 1515, 1495, 1370 , $1270,1205,895,815,735,550,515 \mathrm{~cm}^{-1}$. Exact mass ESI-MS $\mathrm{C}_{18} \mathrm{H}_{20} \mathrm{OC}_{5} \mathrm{H}_{5} \mathrm{NH}$ calculated: 332.2014 found: 332.1992 .

2.23 Synthesis of 1c

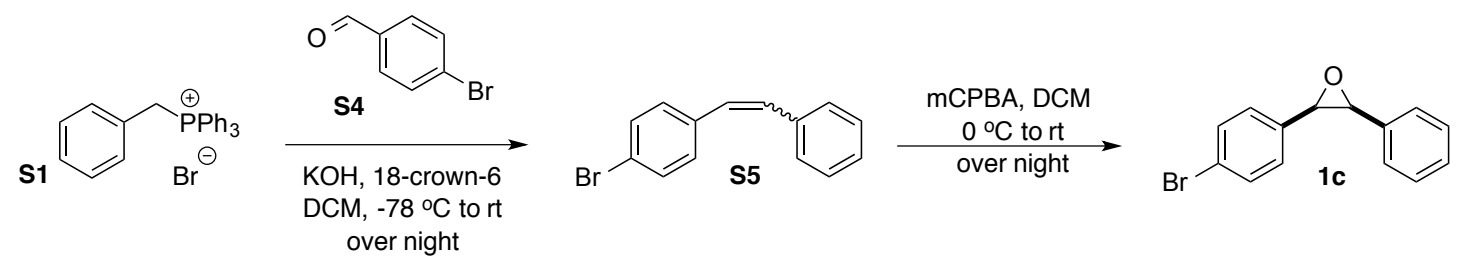

According to general procedure 2, S4 $(2.04 \mathrm{~g}, 11.0 \mathrm{mmol})$ was transformed into S5.

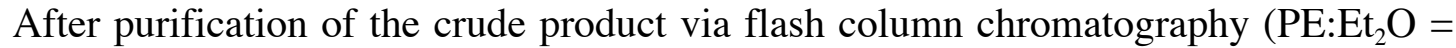
90:10, $\left.\mathrm{R}_{F}=0.62\right) \mathbf{S 5}$ was obtained as a colorless oil (1.24 g, $4.77 \mathrm{mmol}, 48 \%, E: Z=$ 32 : 68) that crystallized upon storing between $5-7{ }^{\circ} \mathrm{C}$. NMR data matches with those reported in literature $\left(\mathrm{E}^{[\mathrm{S7]}}\right.$ and $\left.\mathrm{Z}^{[\mathrm{S8}]}\right)$.

According to general procedure $2, \mathbf{S 5}(1.24 \mathrm{~g}, 4.77 \mathrm{mmol})$ was stirred with $m$ CPBA (70-75\% in water, $1.61 \mathrm{~g}$ ) in $\mathrm{CH}_{2} \mathrm{Cl}_{2}(30 \mathrm{~mL})$. After purification of the crude product via flash column chromatography $\left(\mathrm{PE}: \mathrm{Et}_{2} \mathrm{O}=95: 5, \mathrm{R}_{F}=0.28\right) 1 \mathrm{c}$ was obtained as a colorless oil (934 mg, $3.40 \mathrm{mmol}, 71 \%$ ) that crystallized upon storing between $5-7$ ${ }^{\circ} \mathrm{C}$. The overall yield is $1.18 \mathrm{~g}, 4.31 \mathrm{mmol}, 90 \%$ and corresponds to a cis:trans ratio of 79:21 with 1c-trans $\left(250 \mathrm{mg}, 0.91 \mathrm{mmol}, 19 \%, \mathrm{R}_{F}=0.47\right.$ in $\left.\mathrm{PE}: \mathrm{Et}_{2} \mathrm{O}=95: 5\right) .{ }^{1} \mathrm{H}$ NMR (400 MHz, $\left.\mathrm{CDCl}_{3}\right): 7.30(\mathrm{~d}, J=8.3 \mathrm{~Hz}, 2 \mathrm{H}), 7.23-7.12(\mathrm{~m}, 5 \mathrm{H}), 7.04(\mathrm{~d}, J=$ $8.3 \mathrm{~Hz}, 2 \mathrm{H}), 4.36(\mathrm{~d}, J=4.2 \mathrm{~Hz}, 1 \mathrm{H}), 4.29(\mathrm{~d}, J=4.2 \mathrm{~Hz}, 1 \mathrm{H}) .{ }^{13} \mathrm{C}-\mathrm{NMR}(100 \mathrm{MHz}$, $\mathrm{CDCl}_{3}$ ): 133.89, 133.47, 130.95, 128.50, 127.91, 127.69, 126.76, 121.53, 59.74, 59.13. IR (neat): 3030, 2995, 1590, 1490, 1450, 1410, 1385, 1360, 1170, 1070, 1010, $890,865,800,735,695,600,540,450 \mathrm{~cm}^{-1}$.

2.24 Synthesis of $\mathbf{1 d}$

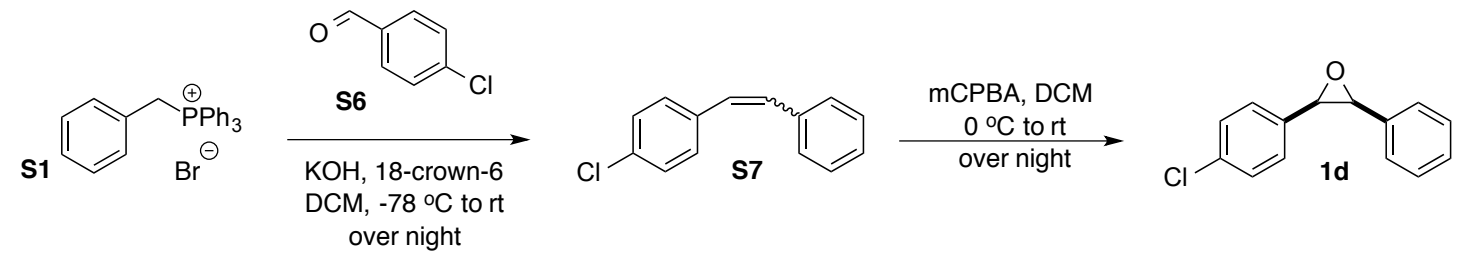

According to general procedure 2, S6 $(1.40 \mathrm{~g}, 10.0 \mathrm{mmol})$ was transformed into $\mathbf{S 7}$. After purification of the crude product via flash column chromatography ( $\mathrm{PE}_{\mathrm{Et}} \mathrm{O}=$ 90:10, $\left.\mathbf{R}_{F}=0.64\right) \mathbf{S} 7$ one was obtained as a colorless oil $(1.25 \mathrm{~g}, 5.81, \mathrm{mmol}, 58 \%, E$ : $Z=42: 58)$. NMR data matches with those reported in literature. ${ }^{[\mathrm{S} 9]}$

According to general procedure $2, \mathbf{S} 7(1.25 \mathrm{~g}, 5.81 \mathrm{mmol})$ was stirred with $m$ CPBA (70-75\% in water, $1.91 \mathrm{~g})$ in DCM $(50 \mathrm{~mL})$. After purification of the crude product via flash column chromatography $\left(\mathrm{PE}: \mathrm{Et}_{2} \mathrm{O}=95: 5, \mathrm{R}_{F}=0.26\right) 1 \mathrm{~d}$ one was obtained as a colorless oil $(862 \mathrm{mg}, 3.75, \mathrm{mmol}, 65 \%)$ that crystalized upon storing between $5-7$ ${ }^{\circ} \mathrm{C}$. The overall yield is $1.17 \mathrm{~g}, 5.10 \mathrm{mmo}, 88 \%$ and corresponds to a cis:trans ratio of

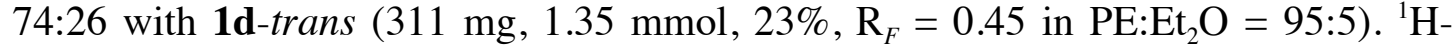


NMR (400 MHz, $\left.\mathrm{CDCl}_{3}\right): 7.23-7.13(\mathrm{~m}, 7 \mathrm{H}), 7.10(\mathrm{~d}, J=8.6 \mathrm{~Hz}, 2 \mathrm{H}), 4.37(\mathrm{~d}, J=$ $4.3 \mathrm{~Hz}, 1 \mathrm{H}), 4.31(\mathrm{~d}, J=4.3 \mathrm{~Hz}, 1 \mathrm{H})$. NMR data matches with those reported in literature. ${ }^{[\mathrm{S} 10]}$

2.25 Synthesis of 1e

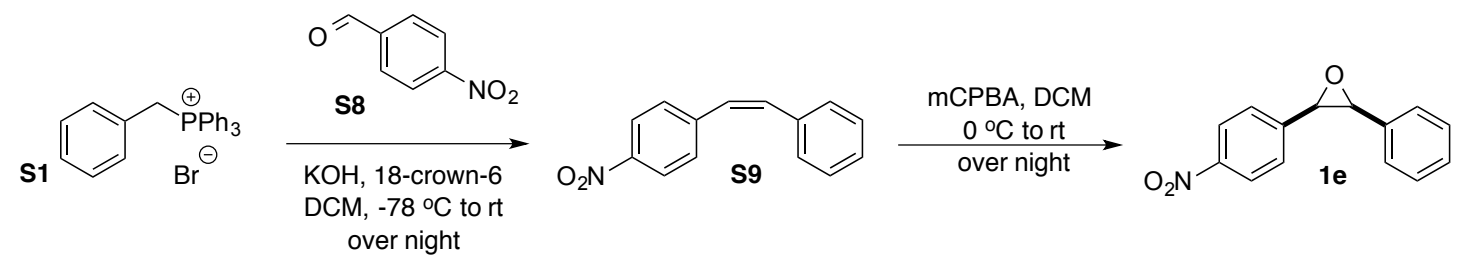

According to general procedure $2, \mathbf{S 8}(1.51 \mathrm{~g}, 10.0 \mathrm{mmol})$ was transformed into $\mathbf{S 9}$. After purification of the crude product $(E: Z=40: 60)$ via flash column chromatography $\left(\mathrm{PE}: \mathrm{Et}_{2} \mathrm{O}=90: 10\right) \mathbf{S 9}$ one was obtained as an orange solid (906 $\mathrm{mg}$, $4.03, \mathrm{mmol}, 40 \%$,). NMR data matches with those reported in literature. ${ }^{[\mathrm{S} 11]}$

According to general procedure $2, \mathbf{S 9}(906 \mathrm{mg}, 5.81 \mathrm{mmol})$ was stirred with $m$ CPBA (70-75\% in water, $1.36 \mathrm{~g})$ in DCM $(30 \mathrm{~mL})$. After purification of the crude product via flash column chromatography $\left(\mathrm{PE}: \mathrm{Et}_{2} \mathrm{O}=80: 20, \mathrm{R}_{F}=0.28\right)$ 1e was obtained as a colorless oil (538 mg, 2.23, mmol, 55\%). ${ }^{1} \mathrm{H}-\mathrm{NMR}$ (400 MHz, $\left.\mathrm{CDCl}_{3}\right): 8.03$ (d, $J=$ $8.6 \mathrm{~Hz}, 2 \mathrm{H}), 7.34(\mathrm{~d}, J=8.5 \mathrm{~Hz}, 2 \mathrm{H}), 7.20-7.14(\mathrm{~m}, 5 \mathrm{H}), 4.47(\mathrm{~d}, J=4.3 \mathrm{~Hz}, 1 \mathrm{H})$, $4.41(\mathrm{~d}, J=4.2 \mathrm{~Hz}, 1 \mathrm{H}) .{ }^{13} \mathrm{C}-\mathrm{NMR}\left(100 \mathrm{MHz}, \mathrm{CDCl}_{3}\right): 147.32,141.92,133.19$, 128.06, 127.98, 127.58, 126.59, 123.05, 60.03, 58.77. IR (neat): 3075, 2990, 1700, $1600,1515,1495,1450,1400,1340,1310,1260,1175,1105,1010,905,850,740$, $695,540,470 \mathrm{~cm}^{-1}$.

2.26 Synthesis of $\mathbf{1 i}$

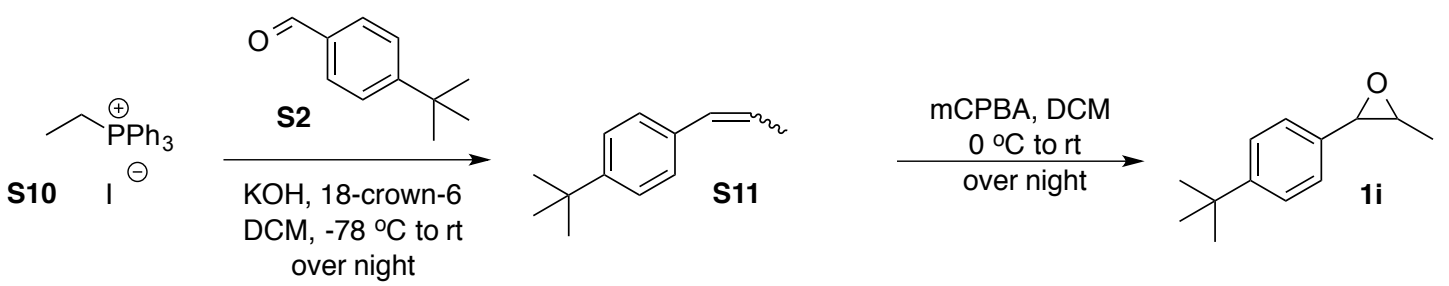

S10 (4.18 g, $10.0 \mathrm{mmol}$ ) was placed with THF ( $80 \mathrm{~mL})$ in a Schlenk-flask under $\mathrm{N}_{2}$. $\mathrm{NaH}(60 \%$ in oil, $400 \mathrm{mg}, 10.0 \mathrm{mmol})$ was added in portions to the mixture. The mixture was allowed to stir $30 \mathrm{~min}$ at room temperature. S2 $(12.3 \mathrm{~g}, 10.0 \mathrm{mmol})$ was added to the mixture. The reaction mixture stirred overnight at room temperature. Water $(40 \mathrm{~mL})$ was added to the mixture. After phase separation and extraction with $\mathrm{PE}$, the combined organics were dried over $\mathrm{MgSO}_{4}$. After purification of the crude product via flash column chromatography $\left(\mathrm{PE}: \mathrm{Et}_{2} \mathrm{O}=90: 10\right)$ S11 was obtained as a colorless oil $(1.35,7.74 \mathrm{mmol}, 77 \%, \mathrm{E}: Z=27: 63)$. ${ }^{1} \mathrm{H}-\mathrm{NMR}\left(400 \mathrm{MHz}, \mathrm{CDCl}_{3}\right)$ : $Z$ S11: $7.37(\mathrm{dd}, J=8.5 \mathrm{~Hz}, J=2.1 \mathrm{~Hz}, 2 \mathrm{H}), 7.29-7.22(\mathrm{~m}, 2 \mathrm{H}), 6.47-6.38(\mathrm{~m}, 1 \mathrm{H})$, $5.76(\mathrm{dq}, J=11.6 \mathrm{~Hz}, J=7.2 \mathrm{~Hz}, 1 \mathrm{H}), 1.92(\mathrm{dd}, J=7.2 \mathrm{~Hz}, J=1.9 \mathrm{~Hz}, 3 \mathrm{H}), 1.34(\mathrm{~s}$, 9H). $\boldsymbol{E}$-S11: $7.34-7.30(\mathrm{~m}, 2 \mathrm{H}), 7.29-7.22(\mathrm{~m}, 2 \mathrm{H}), 6.47-6.38(\mathrm{~m}, 1 \mathrm{H}), 6.20(\mathrm{dq}$, $J=15.7 \mathrm{~Hz}, J=6.6 \mathrm{~Hz}, 1 \mathrm{H}), 1.87(\mathrm{dd}, J=6.6 \mathrm{~Hz}, J=1.7 \mathrm{~Hz}, 3 \mathrm{H}), 1.32(\mathrm{~s}, 9 \mathrm{H}) .{ }^{13} \mathrm{C}-$ NMR $\left(100 \mathrm{MHz}, \mathrm{CDCl}_{3}\right): 149.66,149.82,135.16,134.76,130.78,129.55,128.53$, $125.47,125.34,125.00,124.85,34.48,31.31,18.47,14.69$. IR (neat): 3085, 3020, 
2960, 2905, 2870, 1905, 1785, 1655, 1610, 1515, 1415, 1365, 1270, 1110, 1020, 965 , $945,840,790,710,600,665,455 \mathrm{~cm}^{-1}$.

According to general procedure 2, S11 $(1.35,7.74 \mathrm{mmol})$ was stirred with $m \mathrm{CPBA}$ (70-75\% in water, $2.60 \mathrm{~g}$ ) in $\mathrm{CH}_{2} \mathrm{Cl}_{2}(50 \mathrm{~mL})$. After purification of the crude product via flash column chromatography $\left(\mathrm{PE}: \mathrm{Et}_{2} \mathrm{O}=95: 5, \mathrm{R}_{F}=0.27\right.$ in $\left.\mathrm{PE}: \mathrm{Et}_{2} \mathrm{O}=90: 10\right)$ 1i was obtained as a colorless oil $(956 \mathrm{mg}, 5.03 \mathrm{mmol}, 67 \%$, trans: cis $=26: 74) .{ }^{1} \mathrm{H}$ NMR (400 MHz, $\left.\mathrm{CDCl}_{3}\right)$ : cis-1i: $7.38(\mathrm{~d}, J=8.2,2 \mathrm{H}), 7.23(\mathrm{~d}, J=8.2,2 \mathrm{H}), 4.04(\mathrm{~d}, J$ $=4.1,1 \mathrm{H}), 3.33(\mathrm{p}, J=4.9,1 \mathrm{H}), 1.33(\mathrm{~s}, 9 \mathrm{H}), 1.11(\mathrm{~d}, J=5.4,3 \mathrm{H})$. trans $-1 \mathrm{i}: 7.38(\mathrm{~d}$, $J=8.2,2 \mathrm{H}), 7.21(\mathrm{~d}, J=8.2,2 \mathrm{H}), 3.56(\mathrm{~m}, 1 \mathrm{H}), 3.09-3.03(\mathrm{~m}, 1 \mathrm{H}), 1.45(\mathrm{~d}, J=5.1$, 3H), 1.32 (s, 9H). ${ }^{13} \mathrm{C}-\mathrm{NMR}\left(100 \mathrm{MHz}, \mathrm{CDCl}_{3}\right)$ : cis-1i: 150.40, 132.41, 126.31, 124.90, 57.52, 55.16, 34.54, 31.35, 12.62. trans-1i: 151.14, 134.70, 125.37, 125.33, 59.46, 58.83, 34.57, 31.31, 17.90. IR (neat): 2960, 2905, 2670, 1475, 1515, 1460 , $1360,1215,1125,1205,1060,1020,955,860,830,785,585,550,495 \mathrm{~cm}^{-1}$. $\mathrm{C}_{13} \mathrm{H}_{18} \mathrm{OC}_{5} \mathrm{H}_{5} \mathrm{NH}$ calculated: 270.1858 found: 270.1847 .

\subsection{Synthesis of $\mathrm{B}(p-\text { tol })_{3}$.}

In a 3-neck-flask under nitrogen with reflux condenser were placed magnesium (314 $\mathrm{mg}, 12.9 \mathrm{mmol}$ ) and $\mathrm{Et}_{2} \mathrm{O}(5 \mathrm{~mL}) . \mathrm{I}_{2}$ (one bead) and 1,2-dibromoethane (4 droplets) solved in $\mathrm{Et}_{2} \mathrm{O}(2 \mathrm{~mL})$ were added drop wise to the suspension. Bromotoluene $(2.06 \mathrm{~g}$, $12 \mathrm{mmol}$ ) was dissolved in $10 \mathrm{~mL} \mathrm{Et}{ }_{2} \mathrm{O}$ and was added drop wise to the suspension. The reaction mixture was refluxed for $1 \mathrm{~h}$. The reaction mixture was added to a solution of $\mathrm{BF}_{3}{ }^{*} \mathrm{OEt}_{2}(48 \%, 0.74 \mathrm{~mL})$ in $15 \mathrm{~mL} \mathrm{Et}{ }_{2} \mathrm{O}$. The reaction mixture was allowed to stir overnight at room temperature. The mixture was filtered under $\mathrm{N}_{2}$ atmosphere. Removel of the solvent from the filtrate under reduced pressure and Schlenk-conditions gave a red-brown powder, which was dissolved in $13 \mathrm{~mL}$ dry toluene and stirred overnight. The mixture was filtered under $\mathrm{N}_{2}$ atmosphere. Toluene was removed under reduced pressure to obtain $\mathrm{B}(\mathrm{p}-\text { tol })_{3}$ as a light brownish solid (510 $\mathrm{mg}, 1.8 \mathrm{mmol}, 45 \%$ based on Bromotoluene). ${ }^{1} \mathrm{H}$ NMR $\left(\mathrm{C}_{6} \mathrm{D}_{6}, 400 \mathrm{MHz}\right): 7.71$ (d, $J=$ $7.8 \mathrm{~Hz}, 6 \mathrm{H}), 7.11(\mathrm{~d}, J=7.8 \mathrm{~Hz}, 6 \mathrm{H}), 2.14(\mathrm{~s}, 9 \mathrm{H})$. NMR data matches with those reported in literature. ${ }^{[\mathrm{S} 12]}$

2.28 Synthesis of $\mathrm{B}\left(\mathrm{C}_{6} \mathrm{~F}_{3}\right)_{2}$ Mes.

$\mathrm{B}\left(\mathrm{C}_{6} \mathrm{~F}_{3}\right)_{2}$ Mes was synthesized as described in literature. ${ }^{[\mathrm{S} 13]}$ 


\section{References}

S1. H. Chen, S. Sanjaya, Y.-F. Wang, Org. Lett., 2013, 15, 212.

S2. I. Sorribes, J. R. Cabrero-Antonino, C. Vincent, K. Junge, M. Beller, J. Am. Chem. Soc., 2015, 137, 13580-13587.

S3. $\quad$ C.-Z. Tao, W.-W. Liu, J.-Y. Sun, Z.-L. Cao, H. Li, Y.-F. Zhang, Synthesis, 2010, $8,1280-1284$.

S4. J. Dörfler, S. Doye, Eur. J. Org. Chem., 2014, 2790-2797.

S5. $\quad$ A. R. Ehle, Q. Zhou, M. P. Watson, Org. Lett., 2012, 14, 1202-1205.

S6. T. N. Gieshoff, A. Welther, M. T. Kessler, M. H. G. Prechtl, A. Jacobi van Wangelin, Chem. Commun., 2014, 50, 2261-2264.

S7. T. Luo, R. Zhang, W. Zhang, X. Shen, T. Umemoto, J. Hu, Org. Lett., 2014, 16, 888-891.

S8. $\quad$ M. Das, D. F. O'Shea, Org. Lett., 2016, 18, 336-339.

S9. W. Huang, S.-H. Zhao, N. Xu, Synthesis, 2015, 47, 359-366.

S10. R. Robiette, M. Conza, V. K. Aggarwal, Org. Biomol. Chem., 2006, 4, 621623.

S11. R. Nishibayashi, T. Kurahasi, S. Matsubara, Synlett, 2014, 25, 1287-1290.

S12. Filippou, A. C.; Chernov, O.; Stumpf, K. W.; Schnakenburg, G. Angew. Chem. Int. Ed., 2010, 49, 3296-3300.

S13. G. Erős, H. Mehdi, I. Pápai, T. R. Rokob, P. Király, G. Tárkáyi, T. Soós, Angw. Chem. Int. Ed., 2010, 49, 6550-6563. 
4. Spectra

Figure S1: ${ }^{1} \mathrm{H}-\mathrm{NMR}$ spectrum of $\mathbf{3 a}$ in $\mathrm{CDCl}_{3}$.

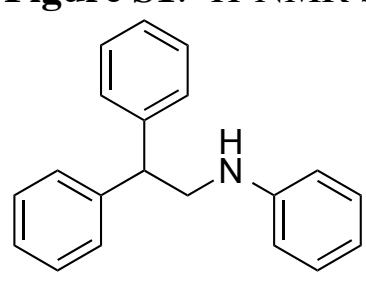

$3 a$

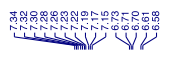

漹
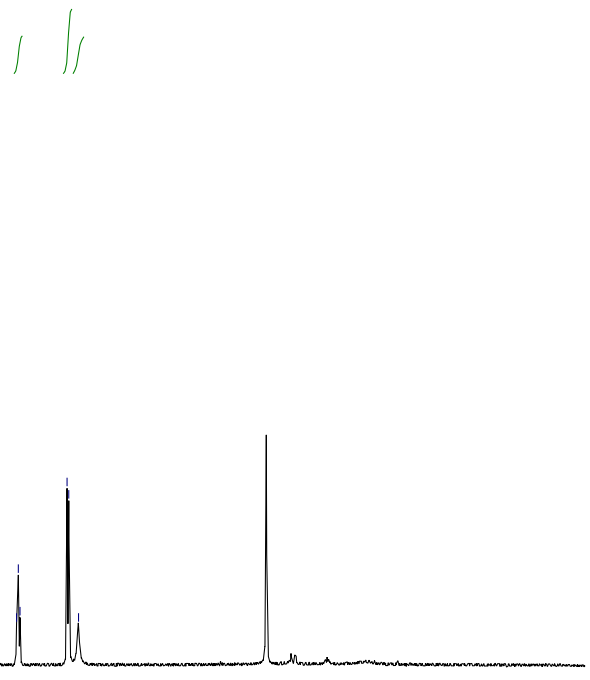

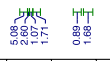

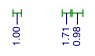

Figure S2: ${ }^{13} \mathrm{C}-\mathrm{NMR}$ spectrum of $\mathbf{3 a}$ in $\mathrm{CDCl}_{3}$.

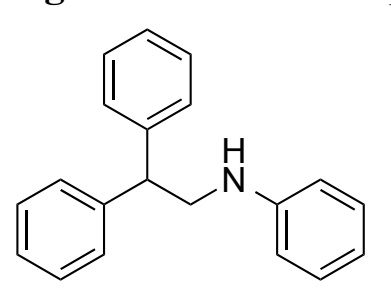

$3 a$

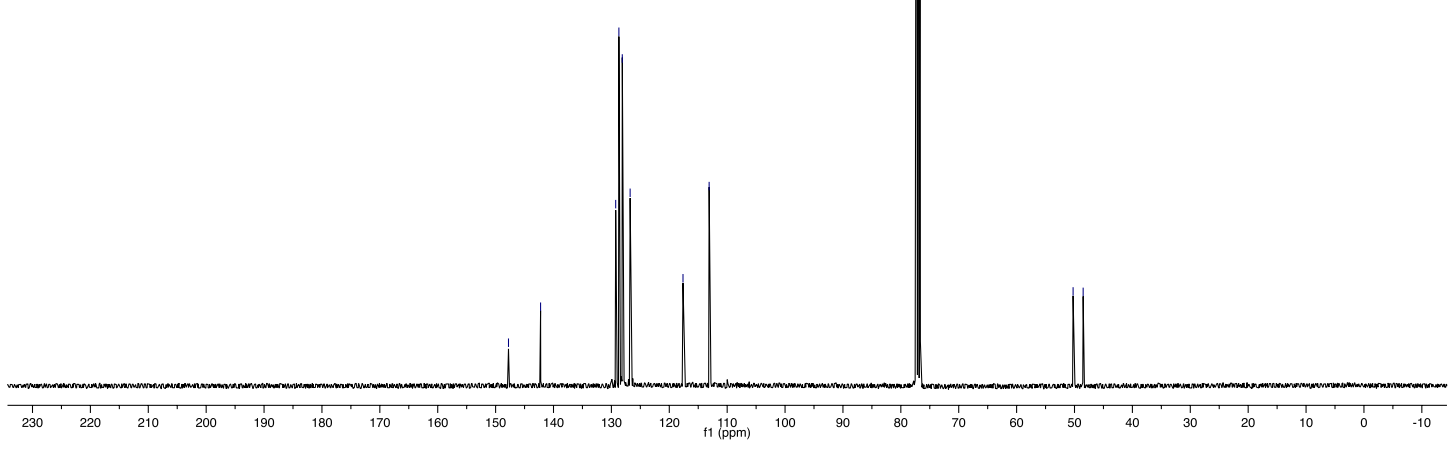

17 
Figure S3: ${ }^{1} \mathrm{H}-\mathrm{NMR}$ spectrum of $\mathbf{3 b}$ in $\mathrm{CDCl}_{3}$.

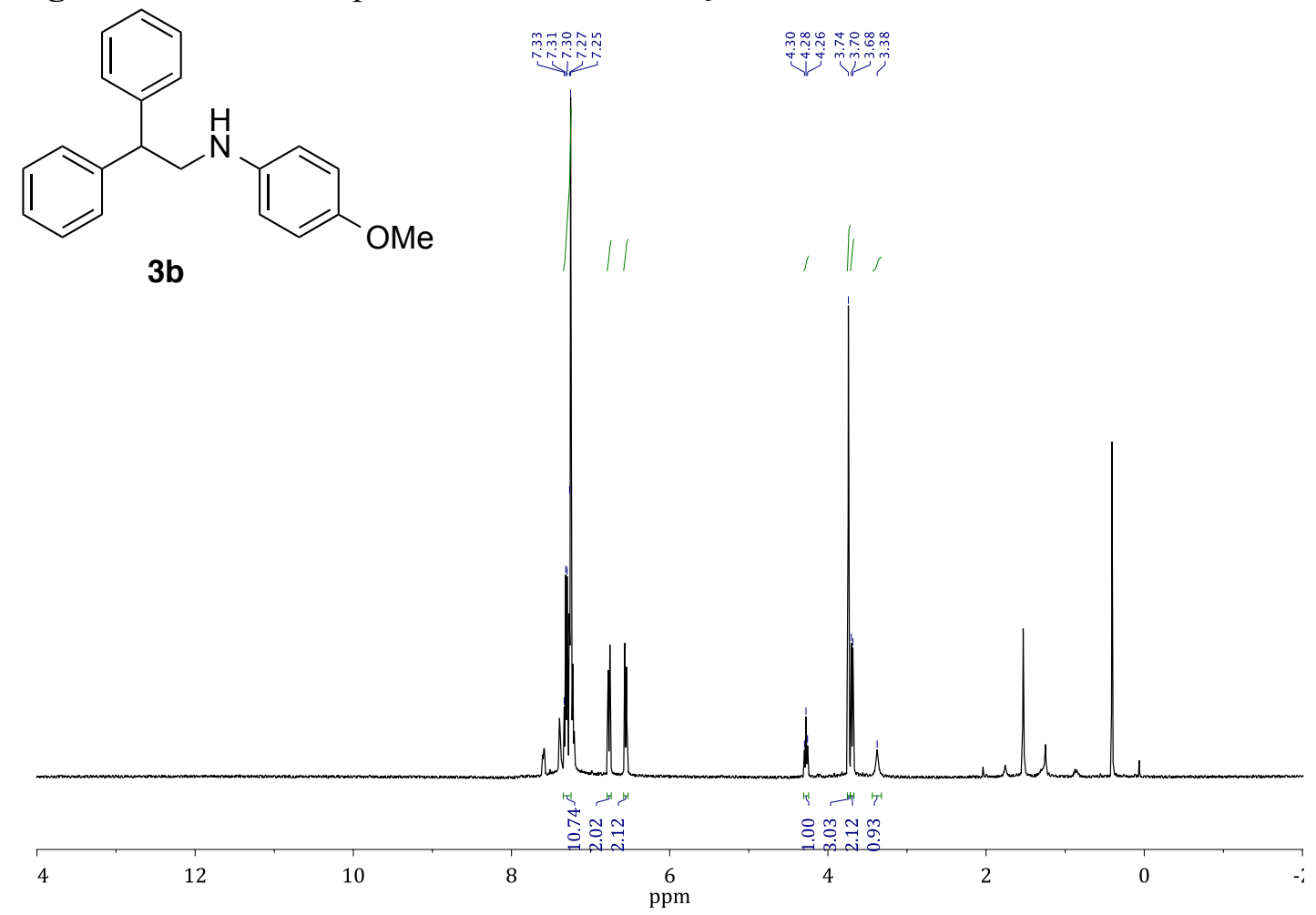

Figure S4: ${ }^{13} \mathrm{C}-\mathrm{NMR}$ spectrum of $\mathbf{3 b}$ in $\mathrm{CDCl}_{3}$

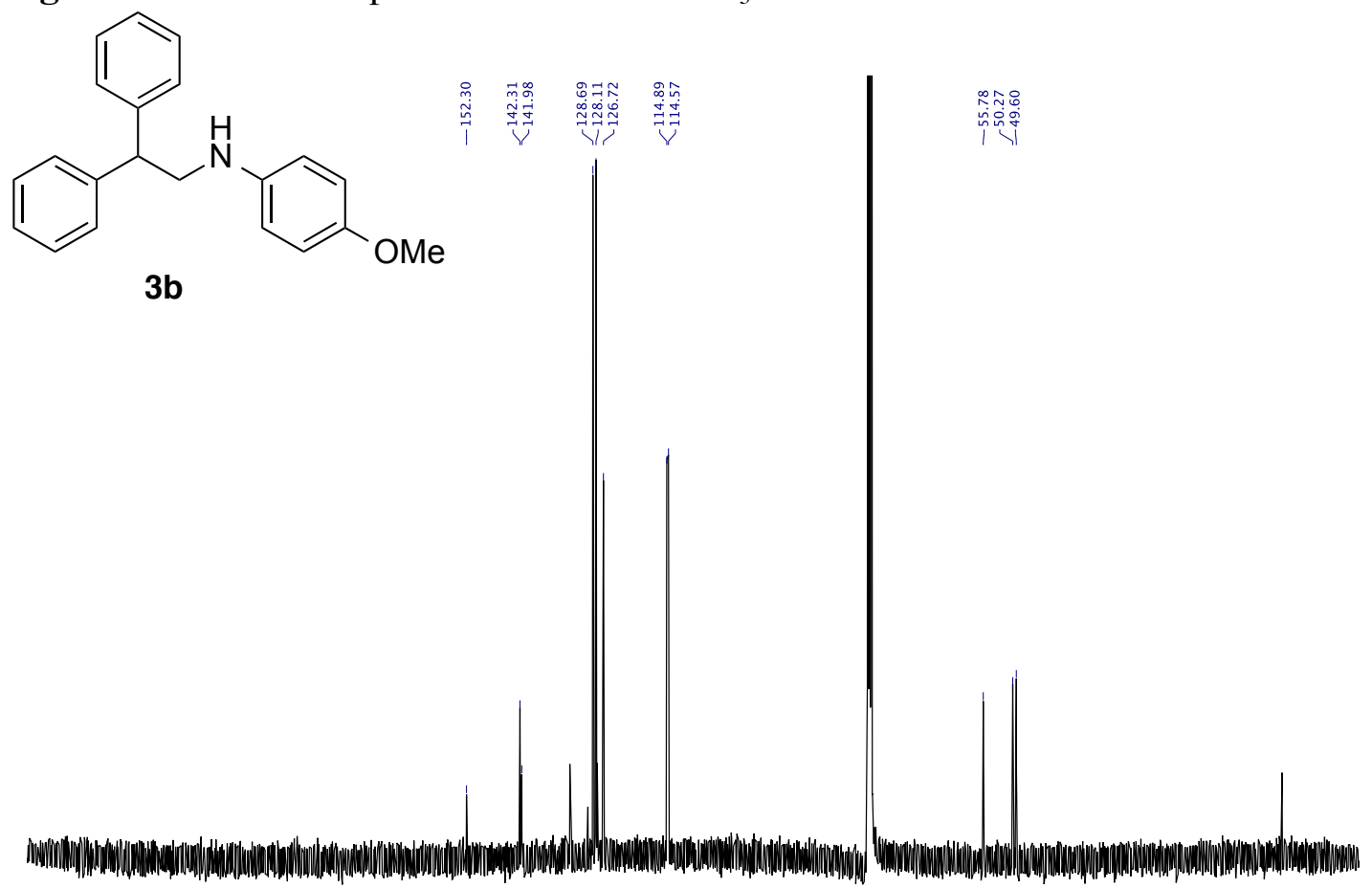

200

150

ppm 100

50

0 
Figure S5: ${ }^{1} \mathrm{H}-\mathrm{NMR}$ spectrum of $\mathbf{3 c}$ in $\mathrm{CDCl}_{3}$.

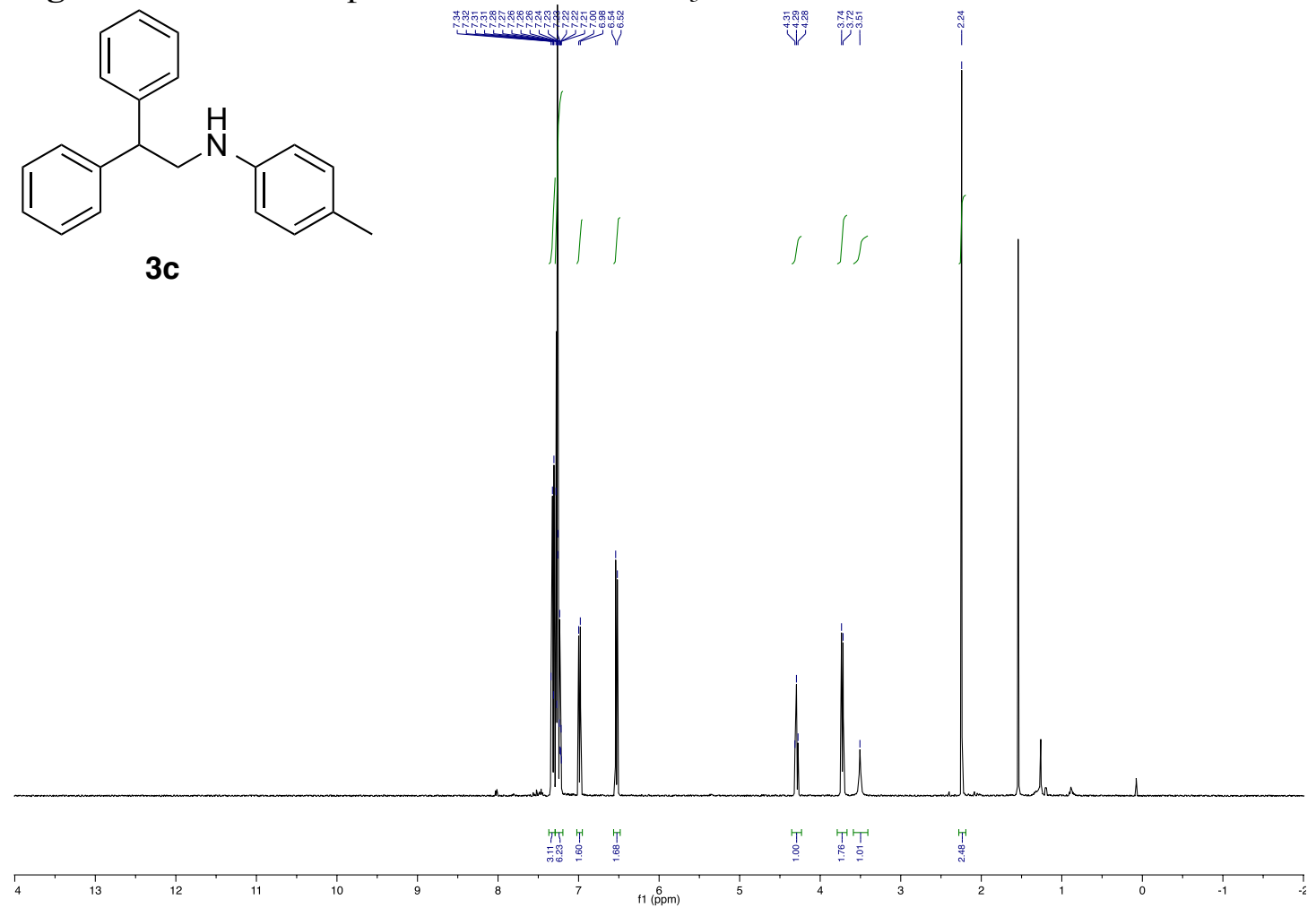

Figure S6: ${ }^{13} \mathrm{C}-\mathrm{NMR}$ spectrum of $\mathbf{3 c}$ in $\mathrm{CDCl}_{3}$.
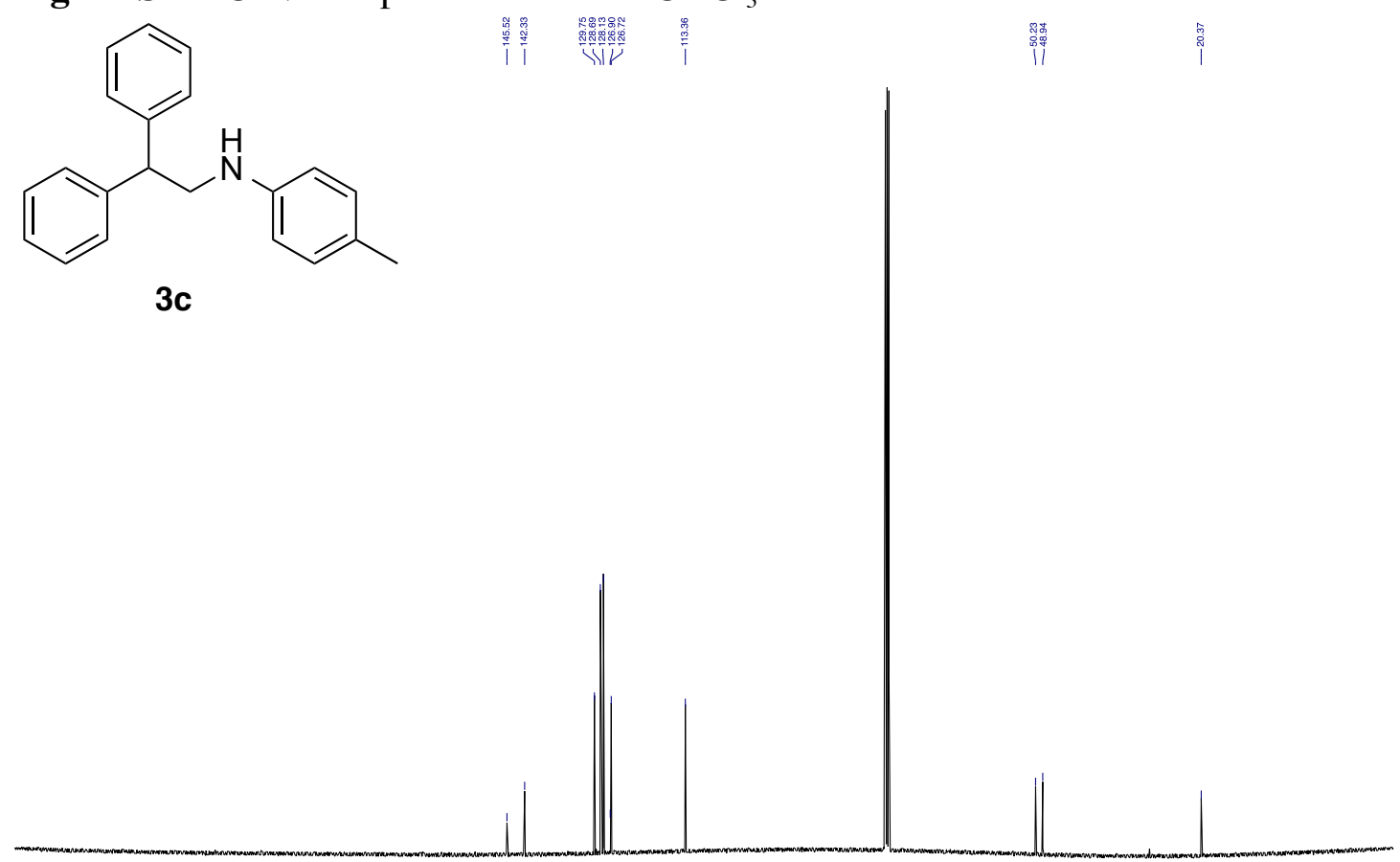
Figure S7: ${ }^{1} \mathrm{H}-\mathrm{NMR}$ spectrum of $\mathbf{3 d}$ in $\mathrm{CDCl}_{3}$.<smiles>Clc1ccc(NCC(c2ccccc2)c2ccccc2)cc1</smiles>

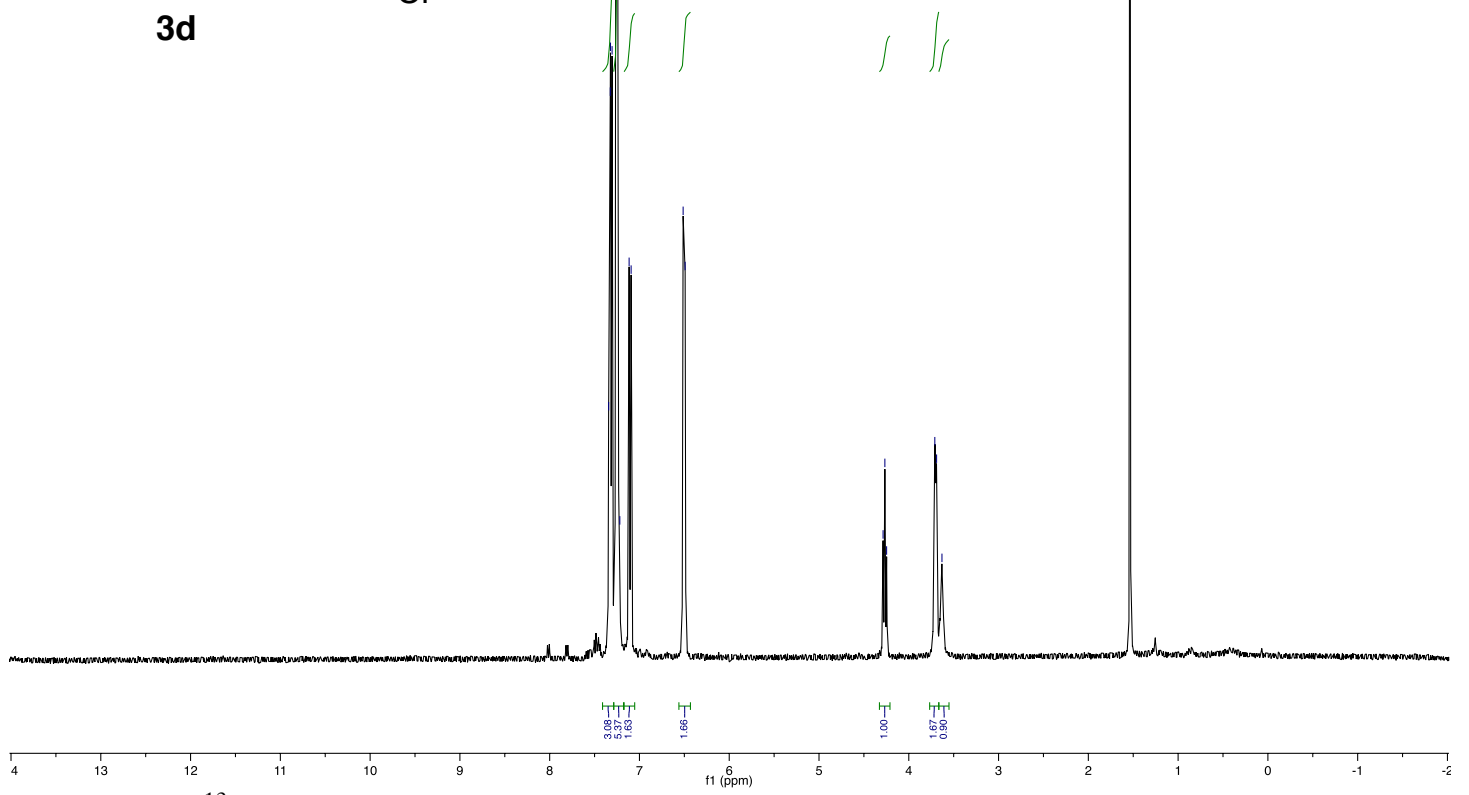

Figure S8: ${ }^{13} \mathrm{C}-\mathrm{NMR}$ spectrum of $\mathbf{3 d}$ in $\mathrm{CDCl}_{3}$.
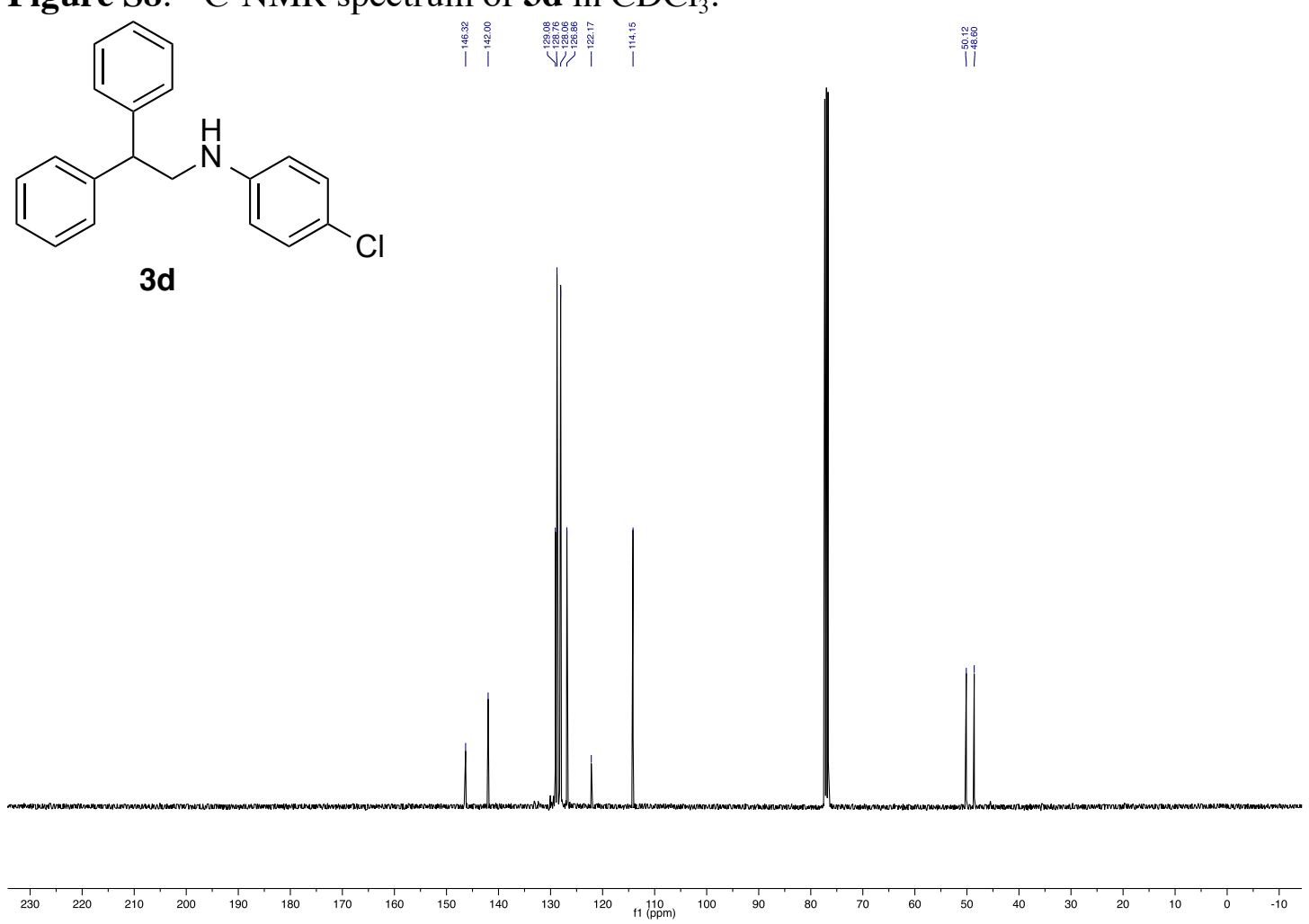

20 
Figure S9: ${ }^{1} \mathrm{H}-\mathrm{NMR}$ spectrum of $\mathbf{3 e}$ in $\mathrm{CDCl}_{3}$.

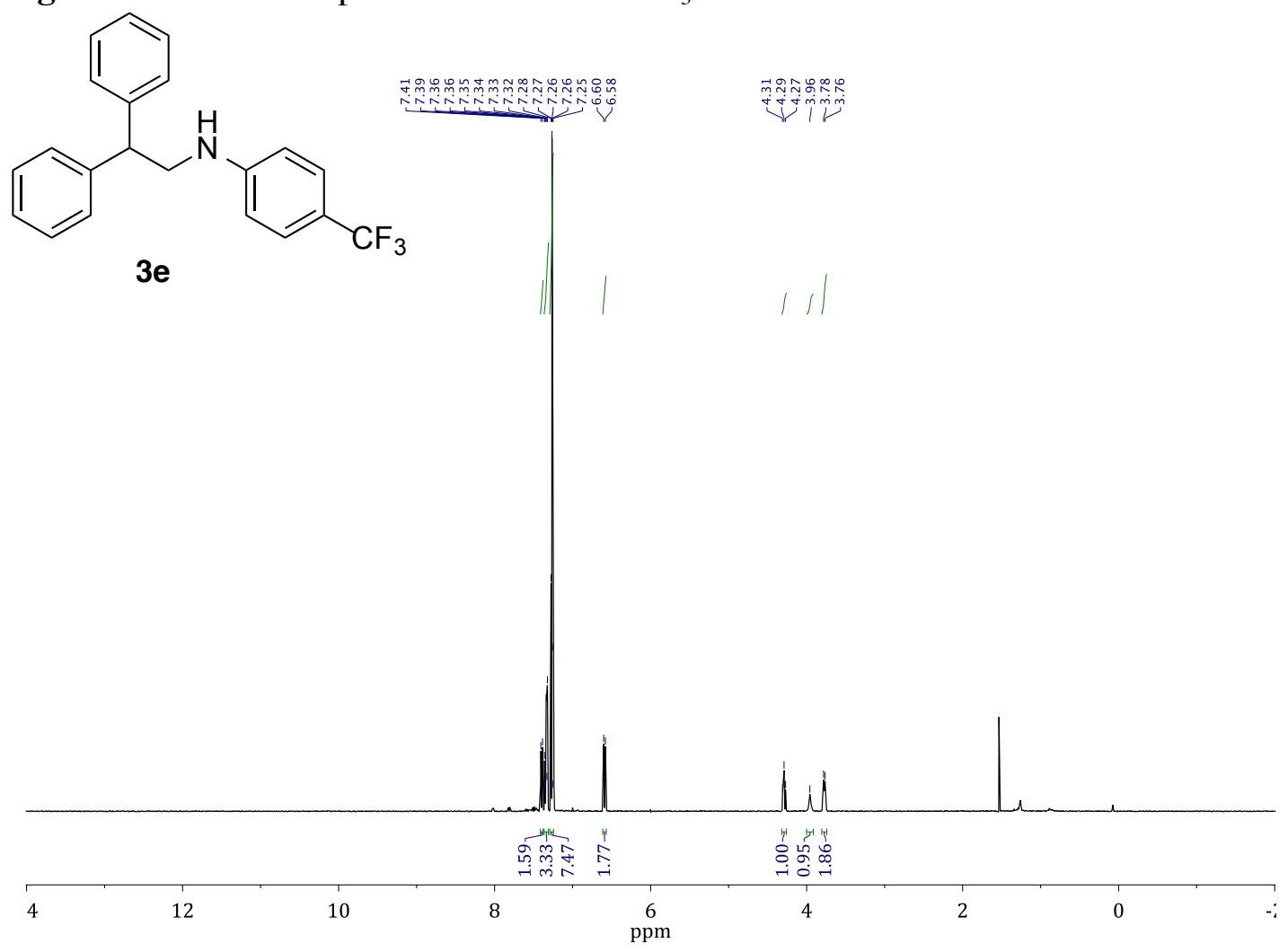

Figure S10: ${ }^{13} \mathrm{C}-\mathrm{NMR}$ spectrum of $\mathbf{3 e}$ in $\mathrm{CDCl}_{3}$.

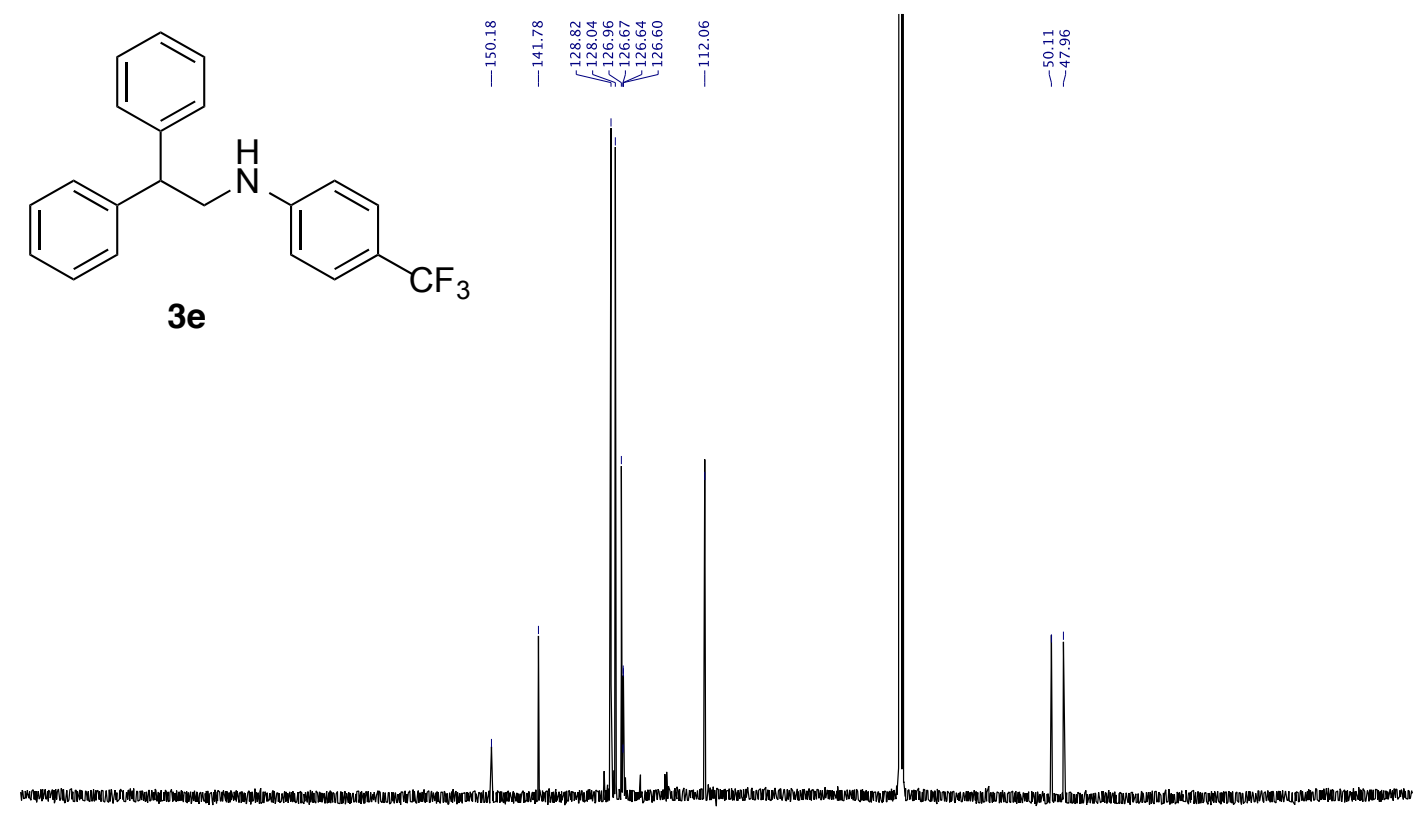

200

150

ppm 100

50

0 
Figure S11: ${ }^{1} \mathrm{H}-\mathrm{NMR}$ spectrum of $\mathbf{3 f}$ in $\mathrm{CDCl}_{3}$.<smiles>CCOC(=O)c1cccc(NCC(c2ccccc2)c2ccccc2)c1</smiles>

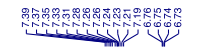

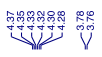

$\frac{\sqrt[3]{9 g h}}{\mathrm{~V}}$

$3 f$
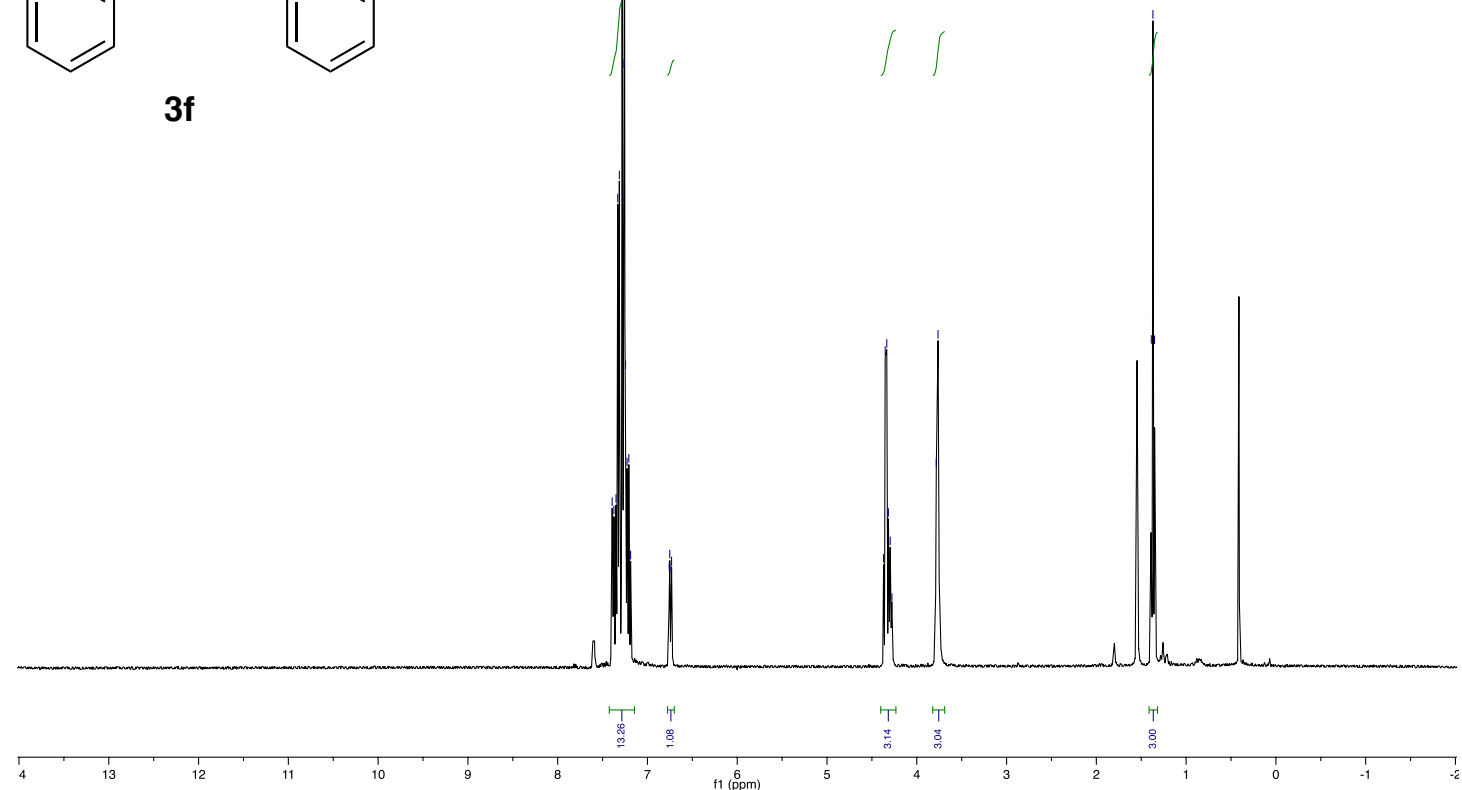

Figure S12: ${ }^{13} \mathrm{C}-\mathrm{NMR}$ spectrum of $\mathbf{3 f}$ in $\mathrm{CDCl}_{3}$.
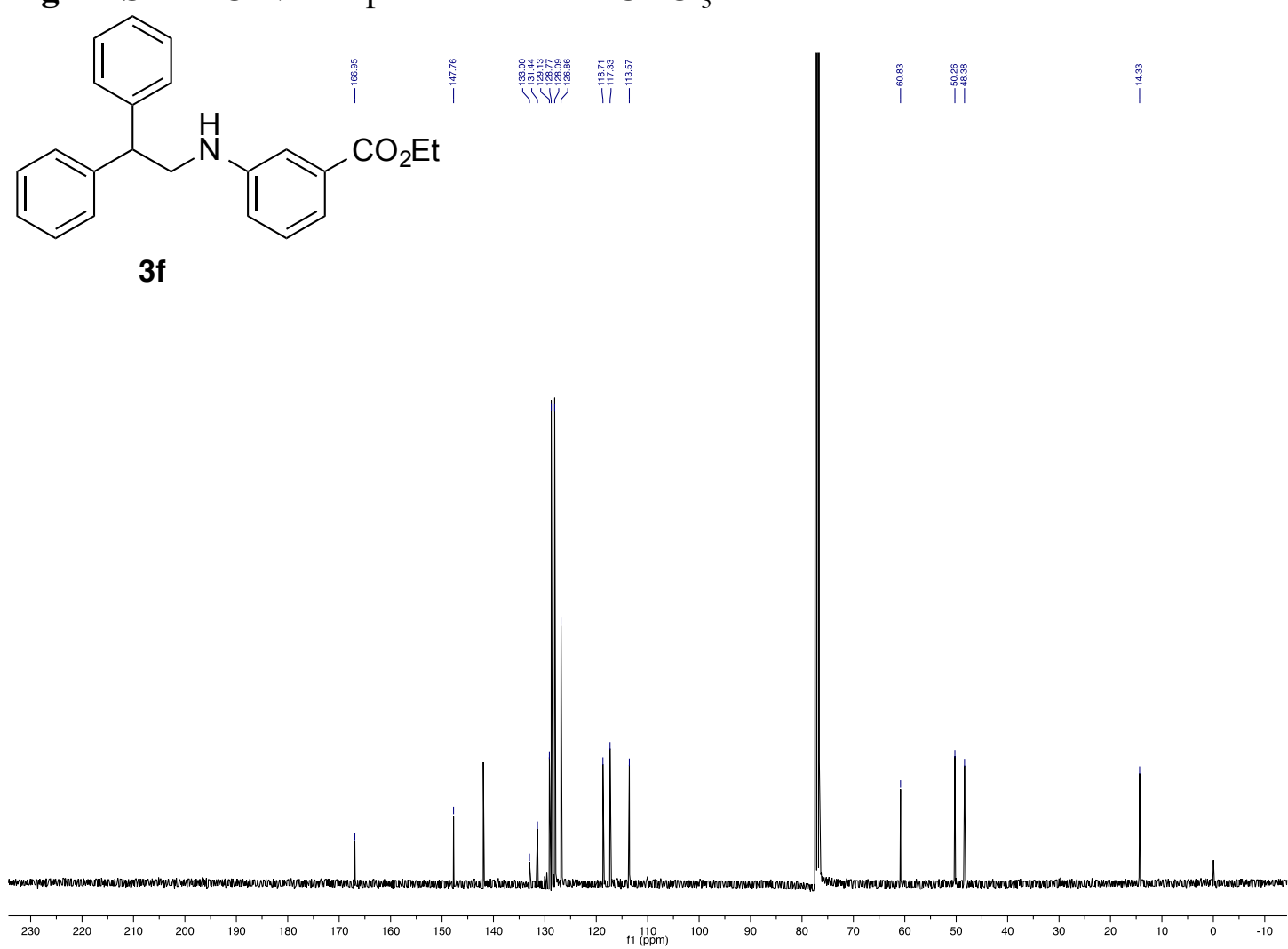

22 
Figure S13: ${ }^{1} \mathrm{H}-\mathrm{NMR}$ spectrum of $\mathbf{3 g}$ in $\mathrm{CDCl}_{3}$.<smiles>Cc1cccc(C)c1NCC(c1ccccc1)c1ccccc1</smiles>

$3 g$

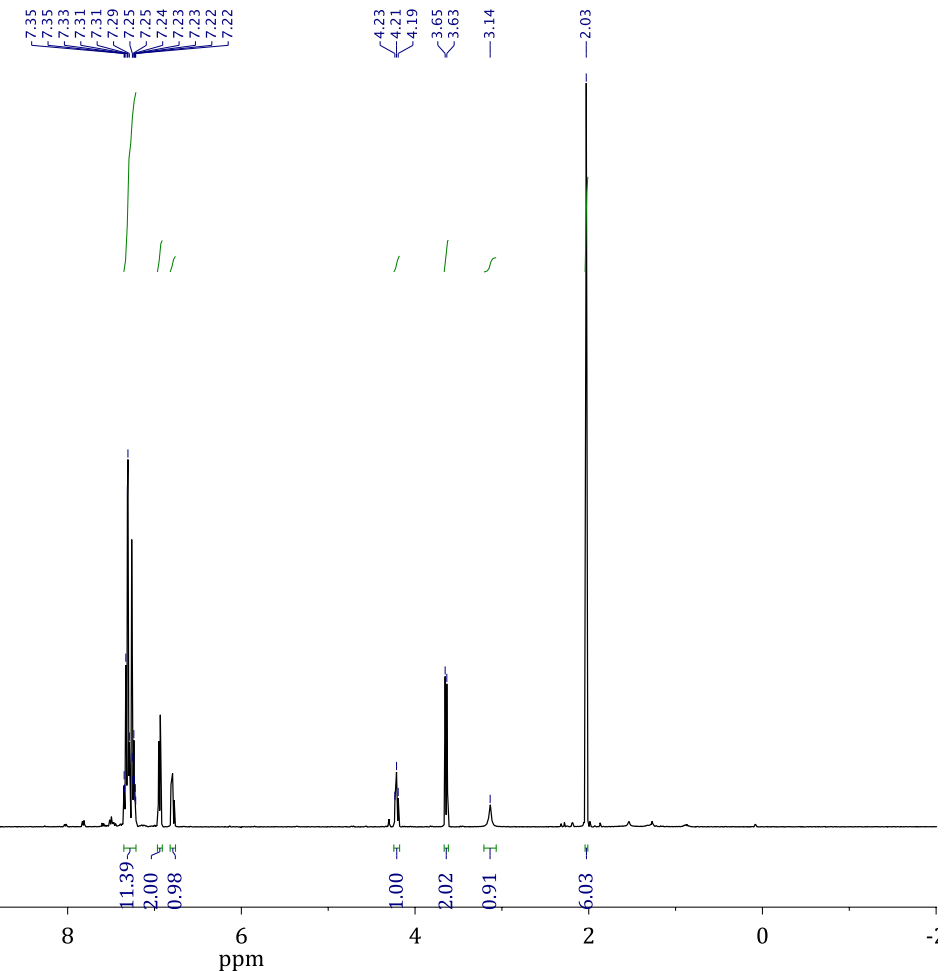

Figure S14: ${ }^{13} \mathrm{C}-\mathrm{NMR}$ spectrum of $3 \mathrm{~g}$ in $\mathrm{CDCl}_{3}$.<smiles>Cc1cccc(C)c1NCC(c1ccccc1)c1ccccc1</smiles>

$3 g$

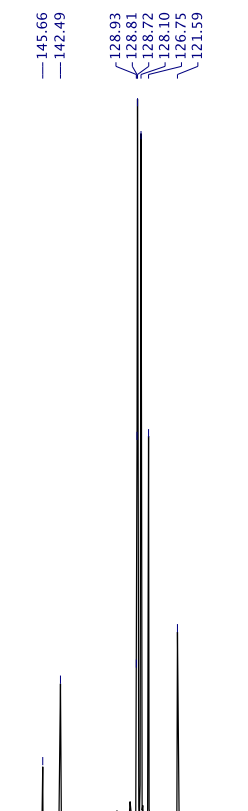


Figure S15: ${ }^{1} \mathrm{H}-\mathrm{NMR}$ spectrum of $\mathbf{3 h}$ in $\mathrm{CDCl}_{3}$.

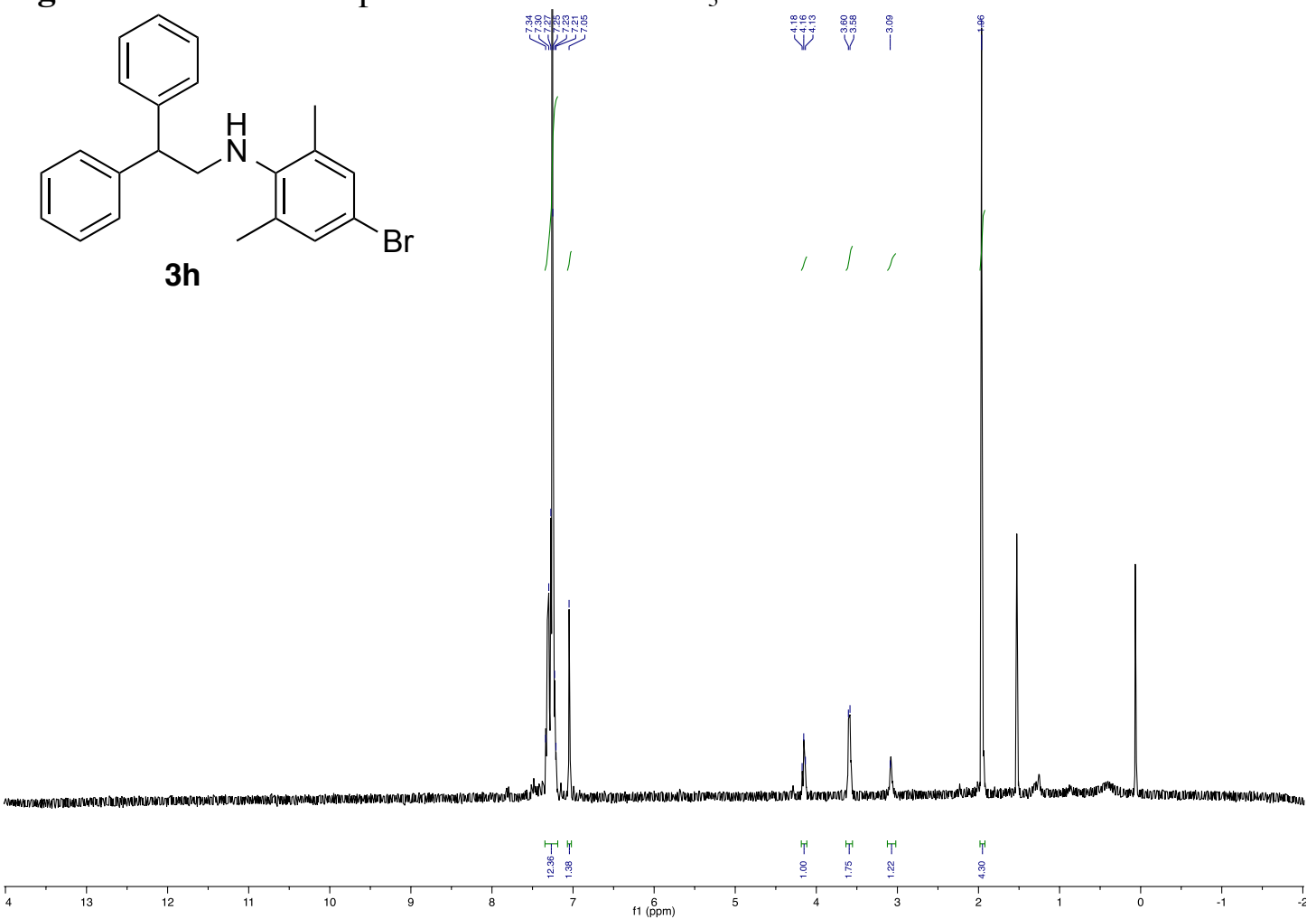

Figure S16: ${ }^{13} \mathrm{C}$-NMR spectrum of $\mathbf{3 h}$ in $\mathrm{CDCl}_{3}$.<smiles>Cc1cc(Br)cc(C)c1NCC(c1ccccc1)c1ccccc1</smiles>

$3 h$

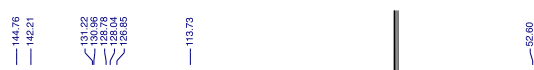

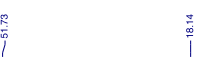

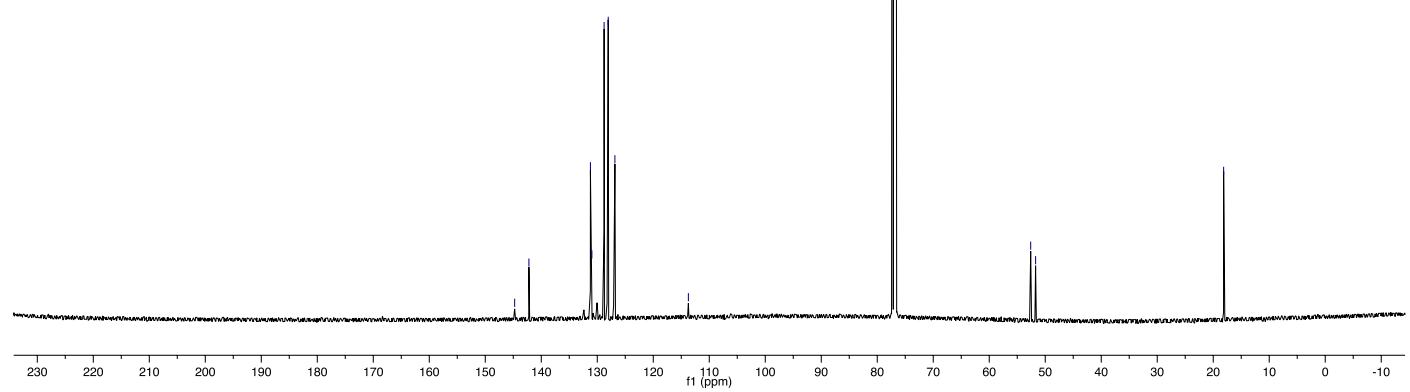


Figure S17: ${ }^{1} \mathrm{H}-\mathrm{NMR}$ spectrum of $\mathbf{3 i}$ in $\mathrm{CDCl}_{3}$.

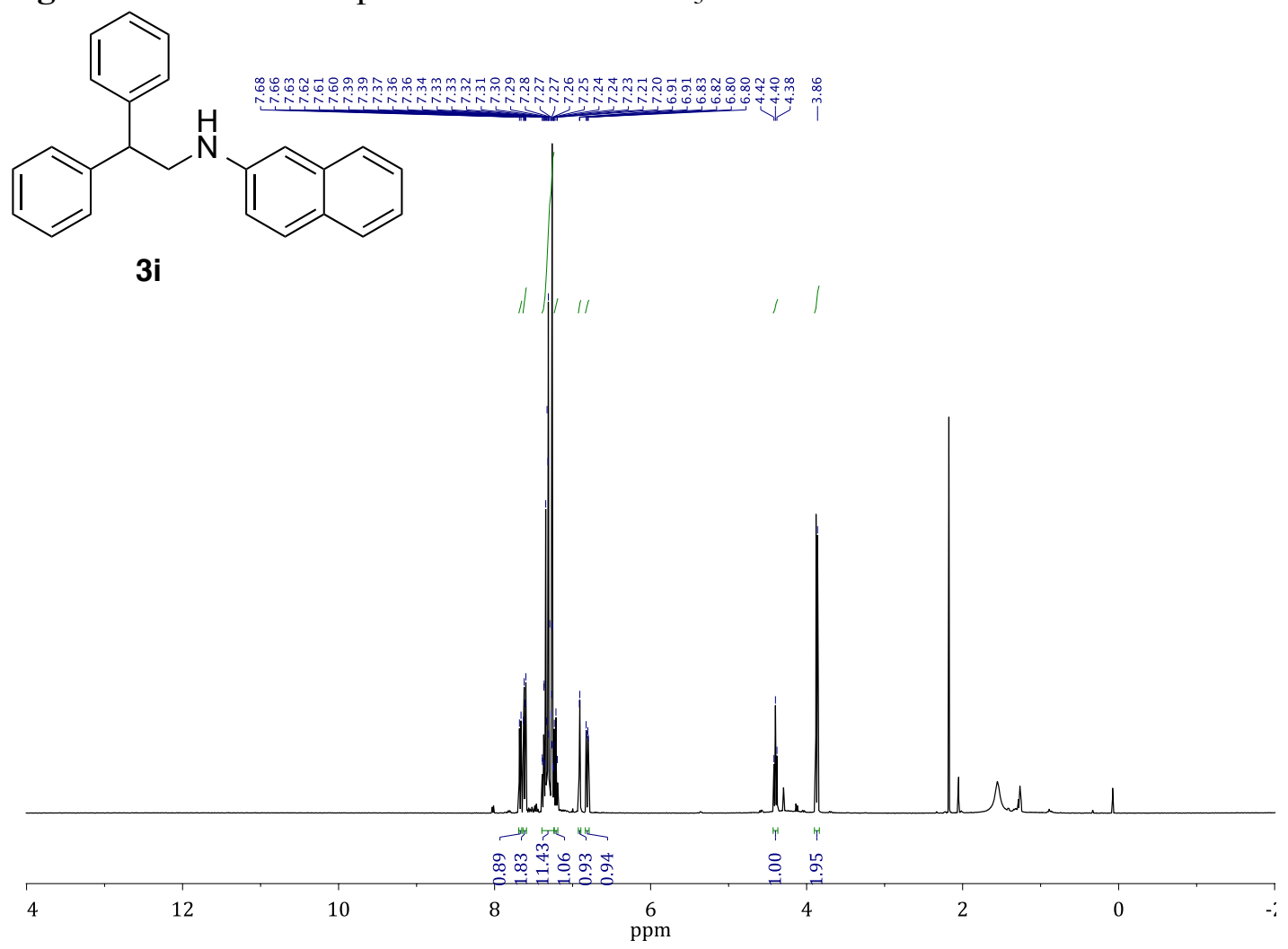

Figure S18: ${ }^{13} \mathrm{C}-\mathrm{NMR}$ spectrum of $\mathbf{3 i}$ in $\mathrm{CDCl}_{3}$.

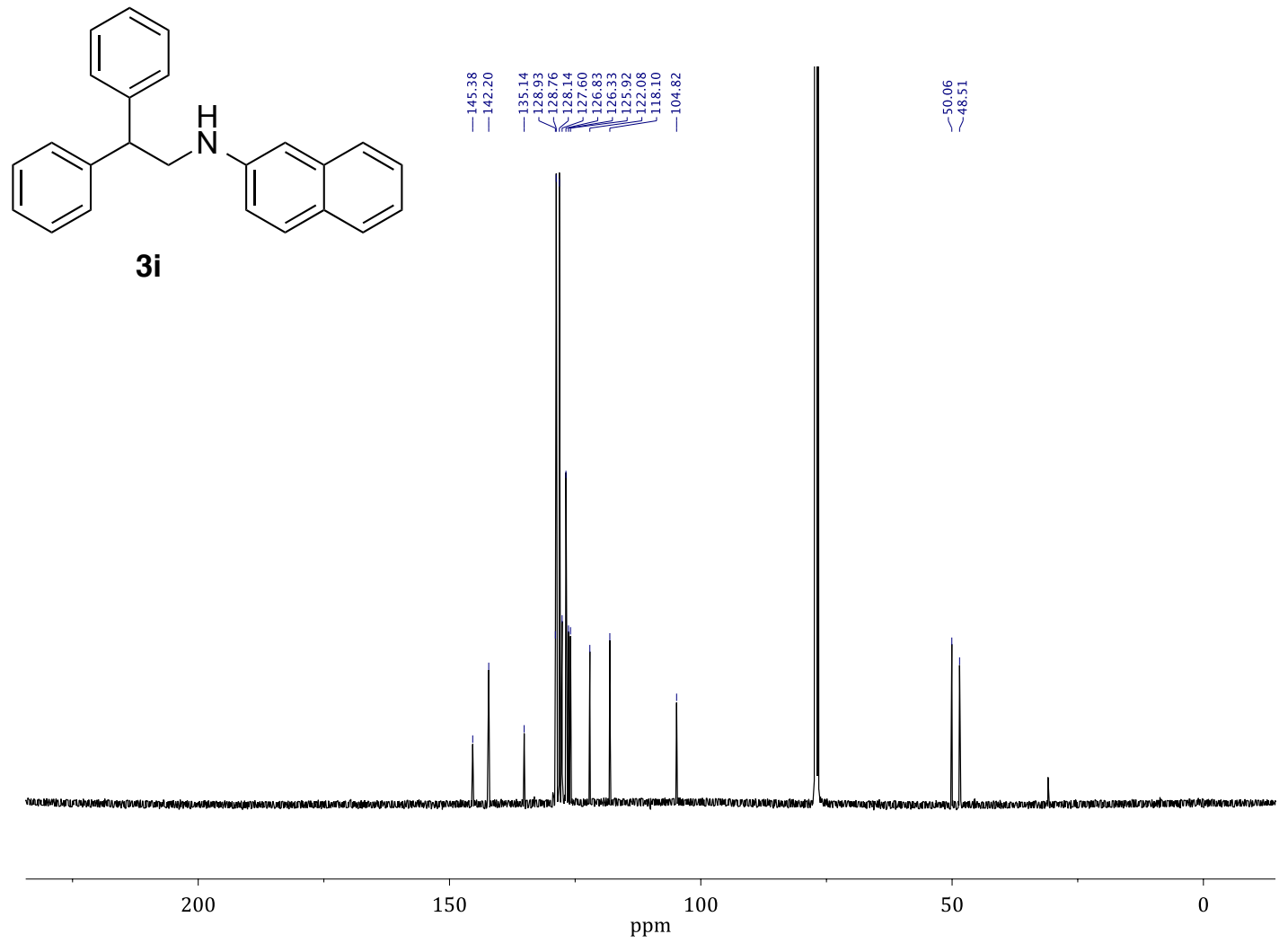


Figure S19: ${ }^{1} \mathrm{H}-\mathrm{NMR}$ spectrum of $\mathbf{3 j}$ in $\mathrm{CDCl}_{3}$.

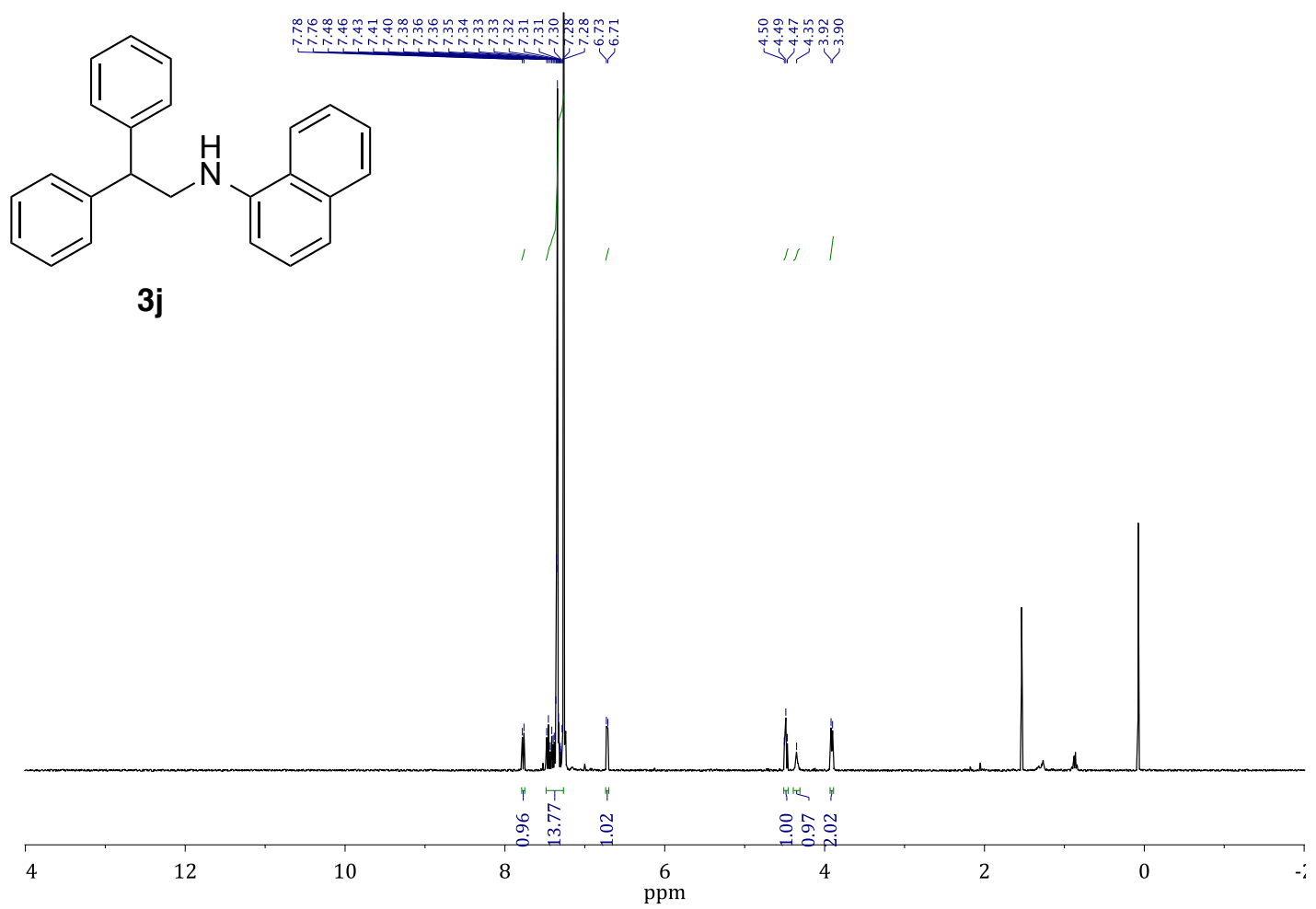

Figure S20: ${ }^{13} \mathrm{C}-\mathrm{NMR}$ spectrum of $\mathbf{3 j}$ in $\mathrm{CDCl}_{3}$.<smiles>c1ccc(C(CNc2cccc3ccccc23)c2ccccc2)cc1</smiles>

3j

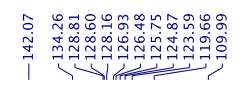

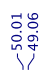

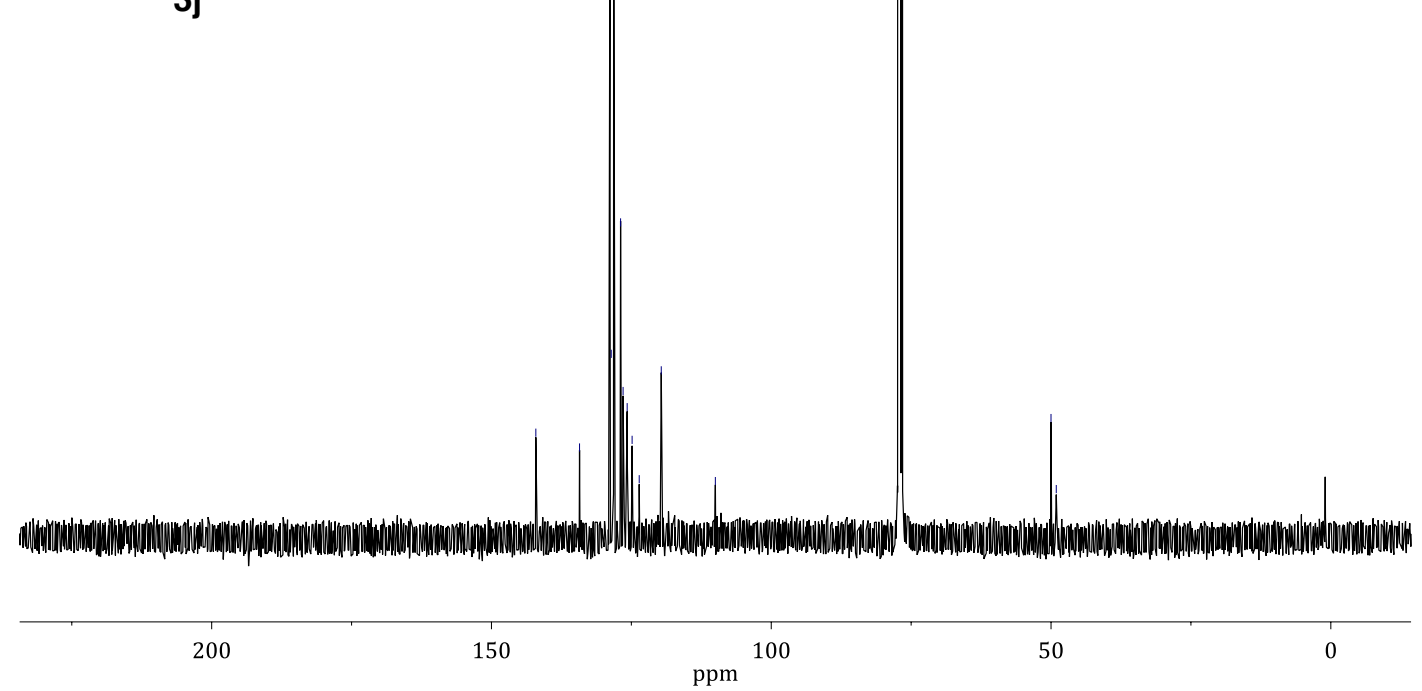


Figure S21: ${ }^{1} \mathrm{H}-\mathrm{NMR}$ spectrum of $\mathbf{3 n}$ in $\mathrm{CDCl}_{3}$.

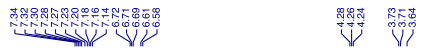
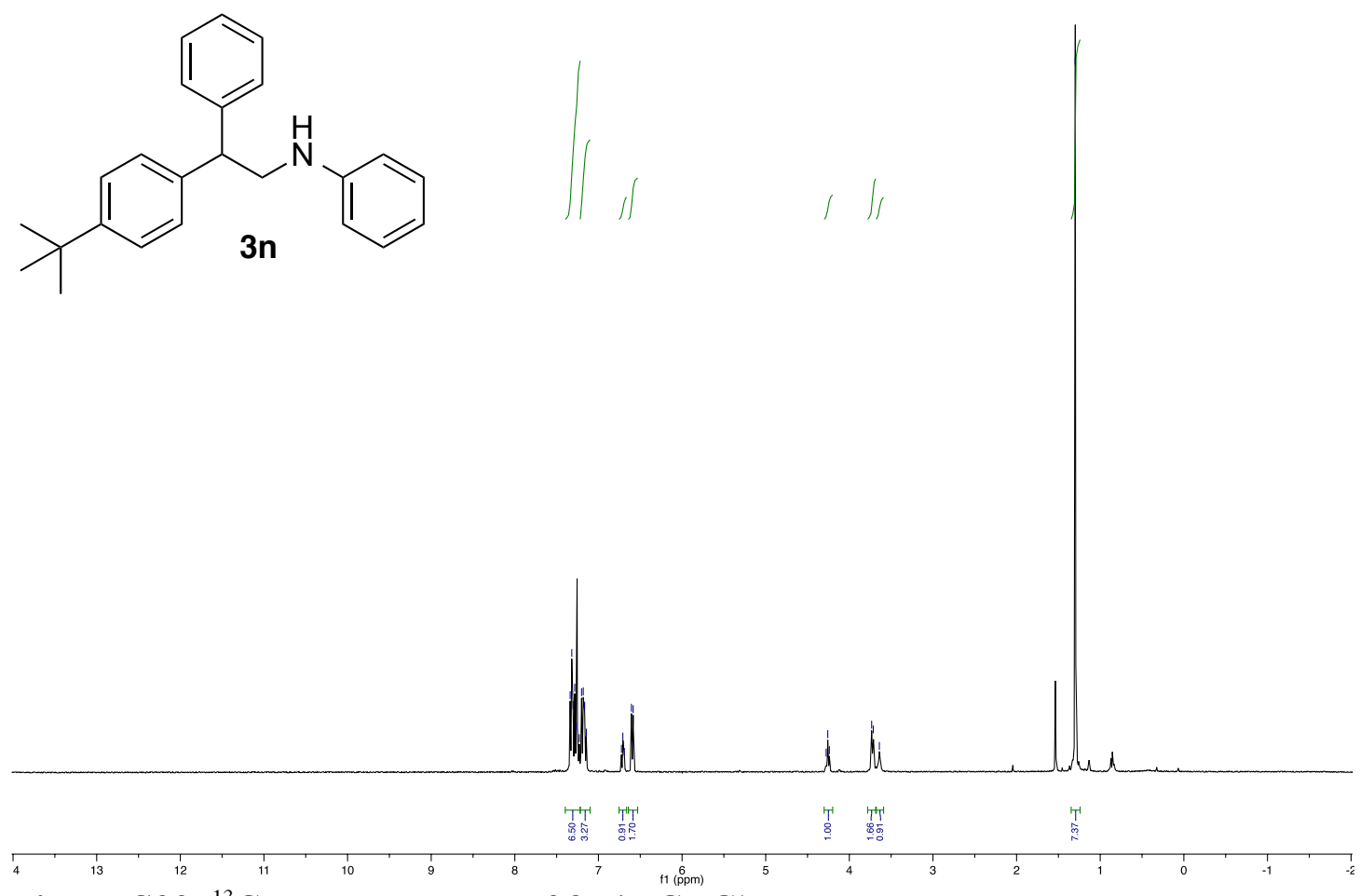

Figure S22: ${ }^{13} \mathrm{C}-\mathrm{NMR}$ spectrum of $\mathbf{3 n}$ in $\mathrm{CDCl}_{3}$.
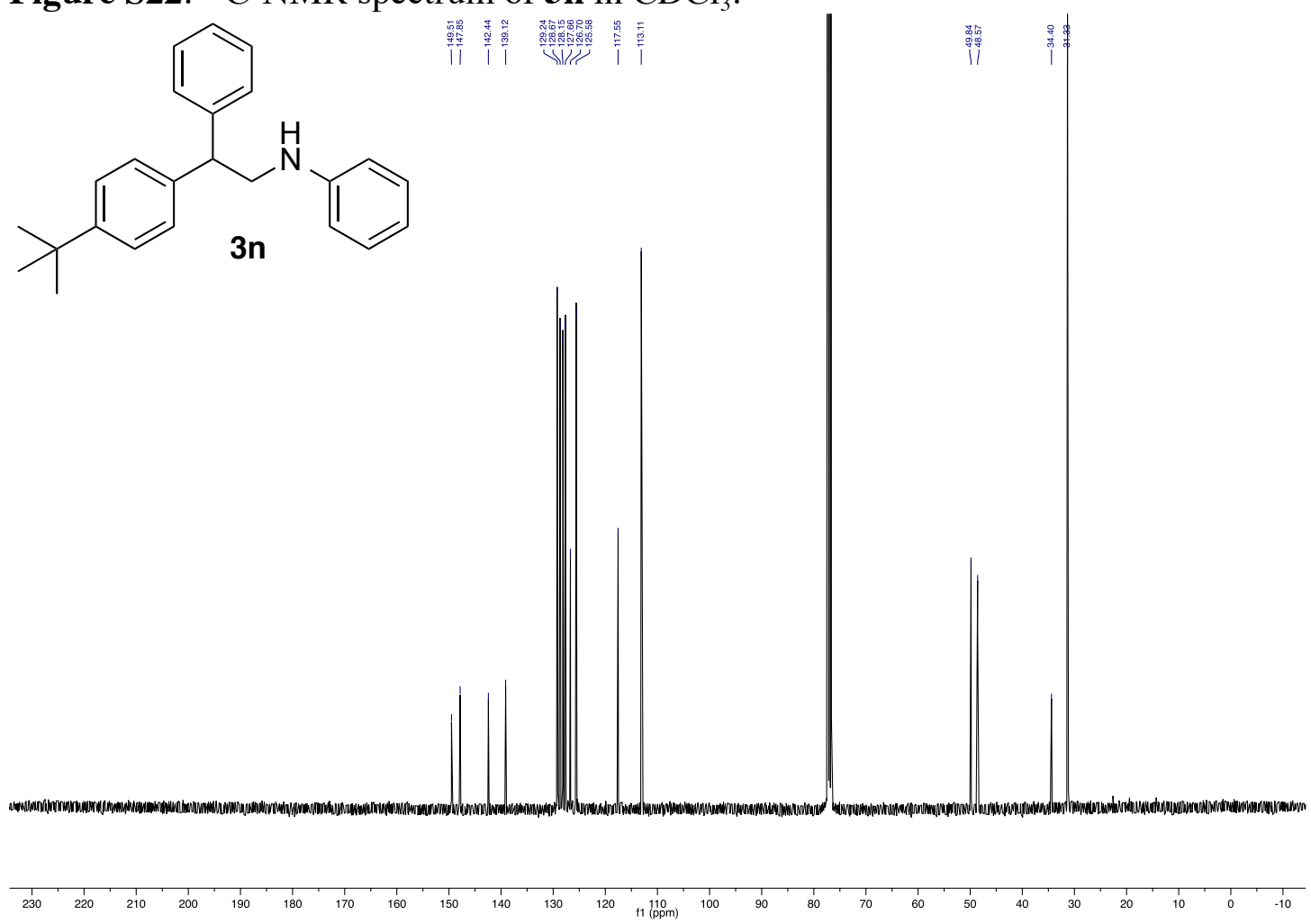

27 
Figure S23: ${ }^{1} \mathrm{H}-\mathrm{NMR}$ spectrum of $\mathbf{3 o}$ in $\mathrm{CDCl}_{3}$.
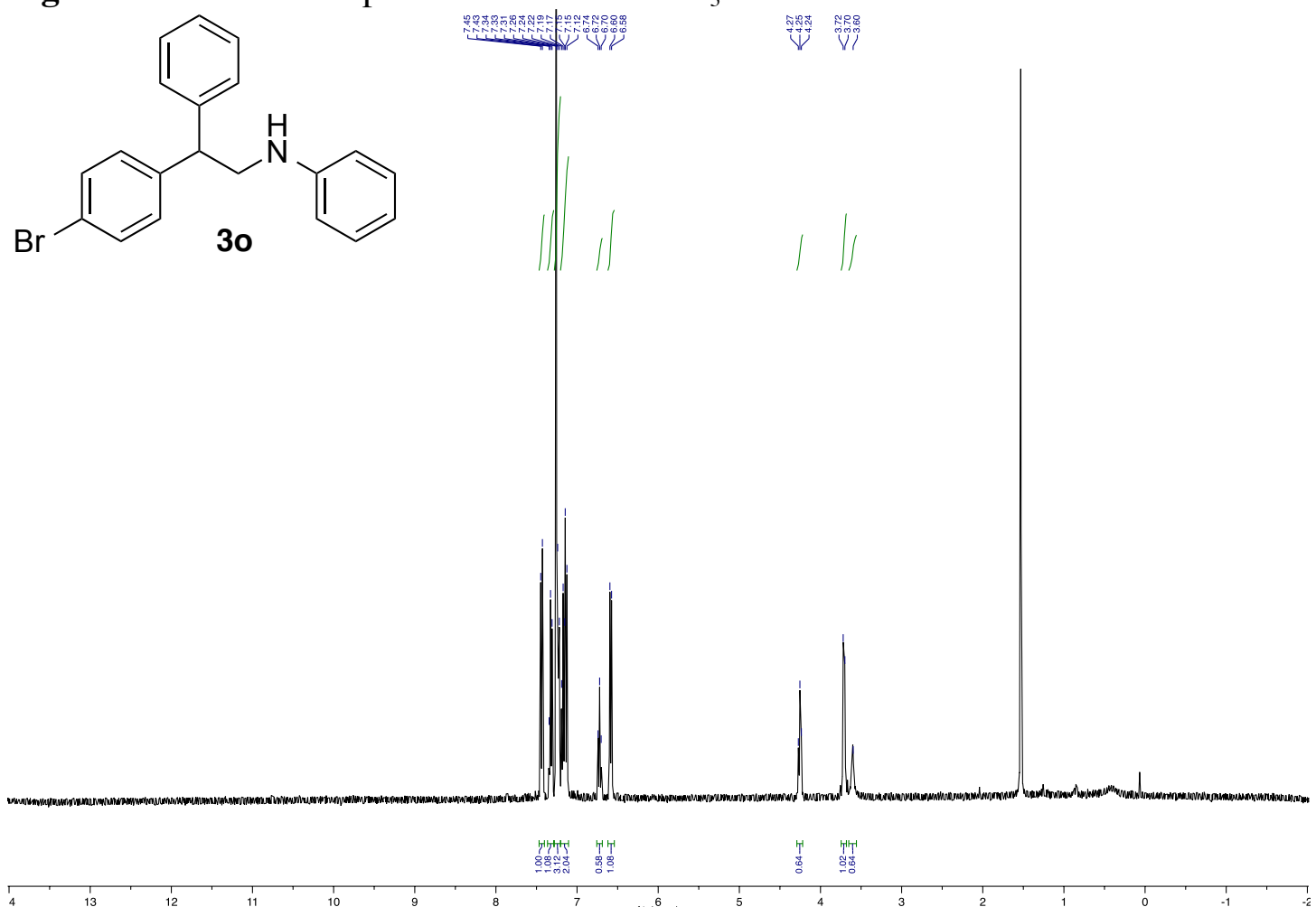

Figure S24: ${ }^{13} \mathrm{C}$-NMR spectrum of 30 in $\mathrm{CDCl}_{3}$.
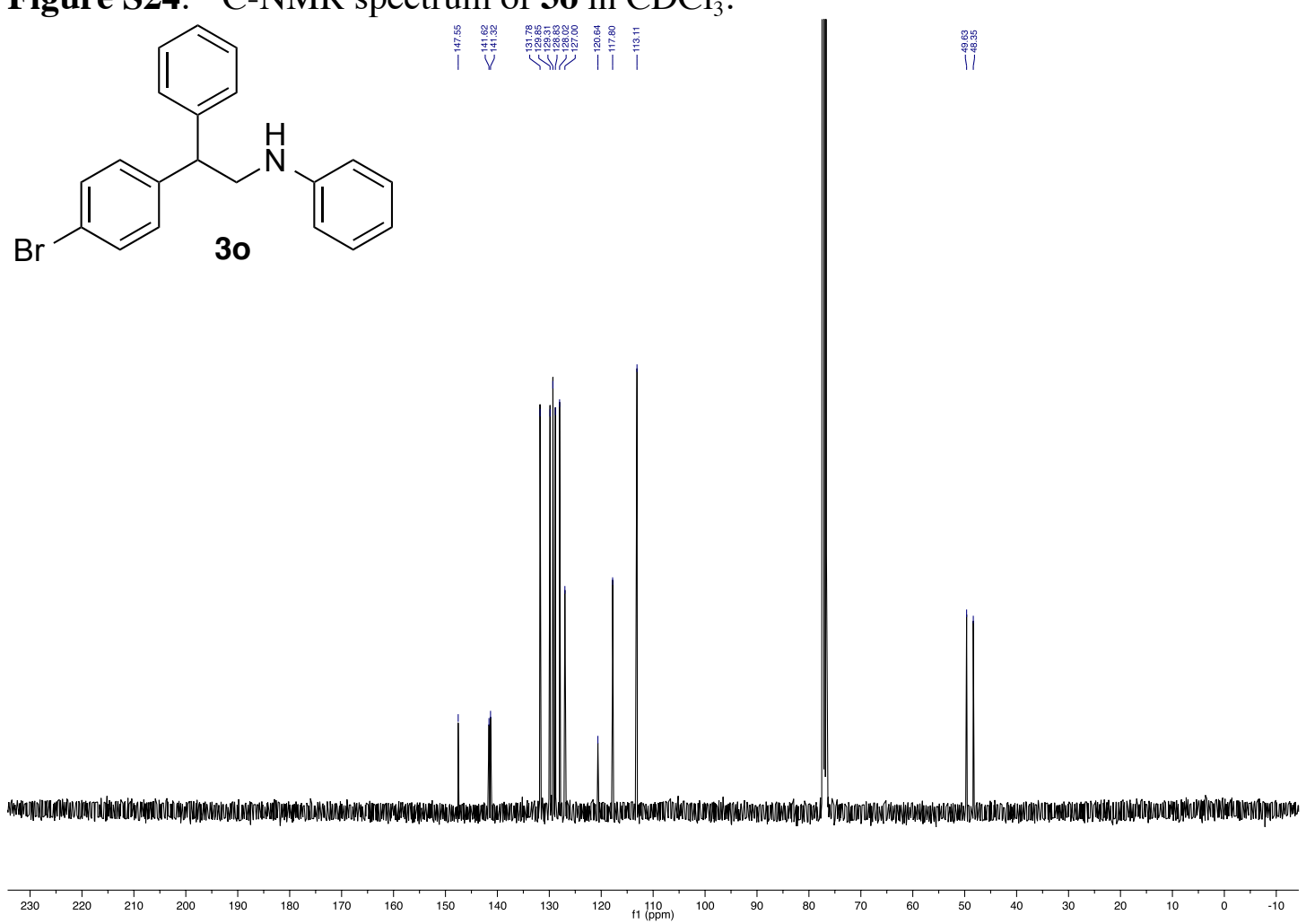

28 
Figure S25: ${ }^{1} \mathrm{H}-\mathrm{NMR}$ spectrum of $\mathbf{3 p}$ in $\mathrm{CDCl}_{3}$.
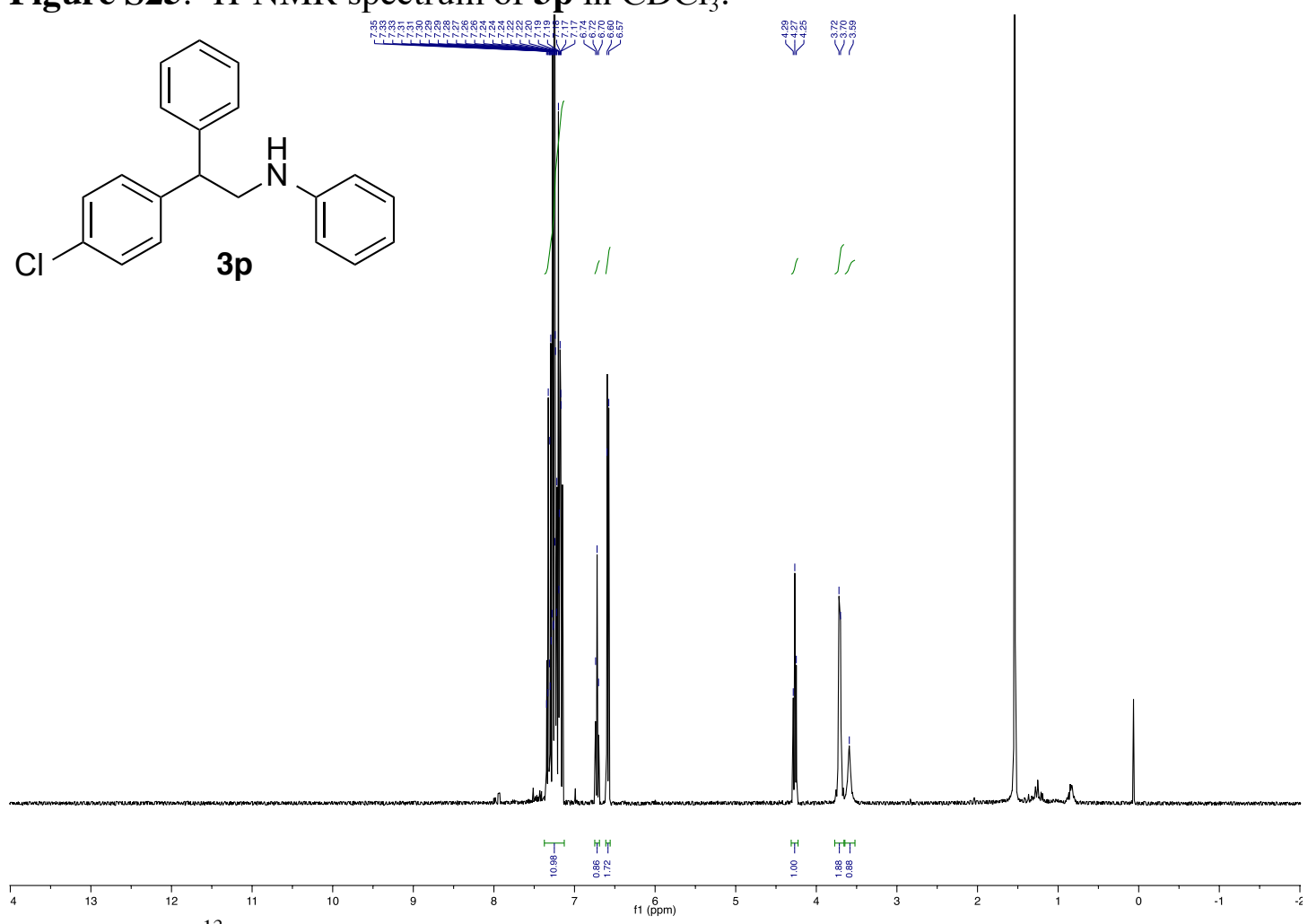

Figure S26: ${ }^{13} \mathrm{C}-\mathrm{NMR}$ spectrum of $\mathbf{3 p}$ in $\mathrm{CDCl}_{3}$.
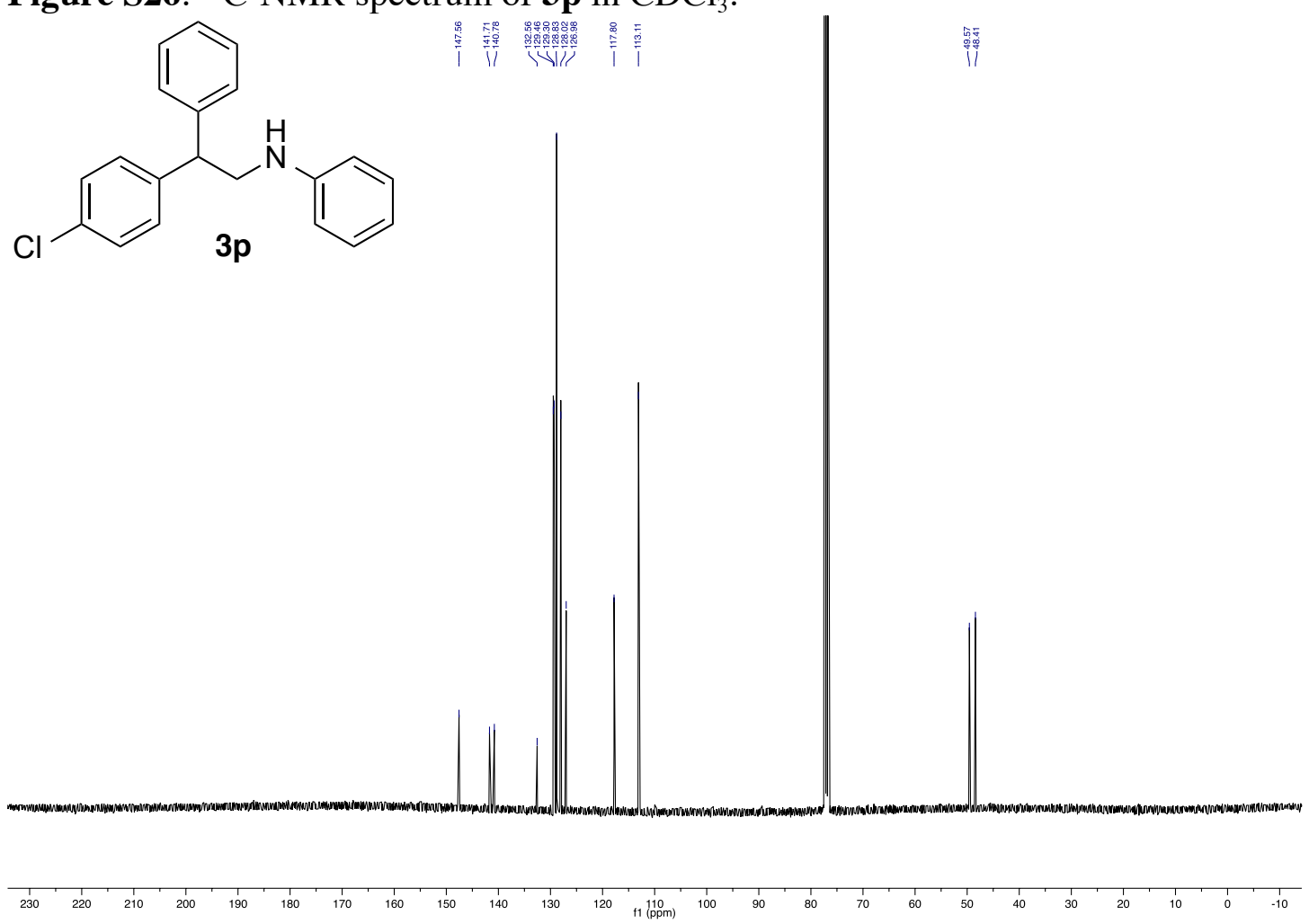

29 
Figure S27: ${ }^{13} \mathrm{C}-\mathrm{NMR}$ spectrum of $\mathbf{3 q}$ in $\mathrm{CDCl}_{3}$.

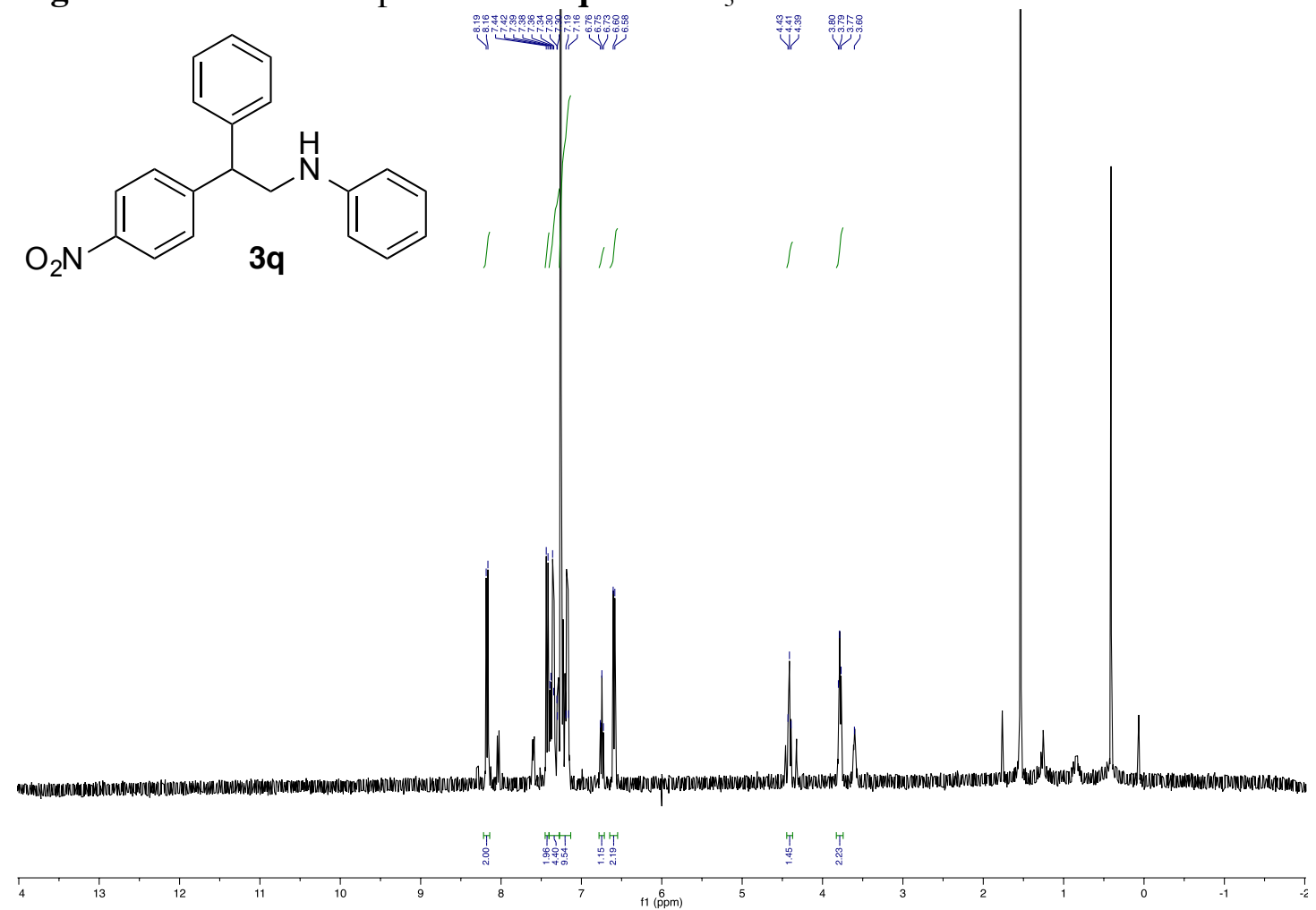

Figure S28: ${ }^{13} \mathrm{C}-\mathrm{NMR}$ spectrum of $\mathbf{3 q}$ in $\mathrm{CDCl}_{3}$.<smiles>O=[N+]([O-])c1ccc(C(CNc2ccccc2)c2ccccc2)cc1</smiles>

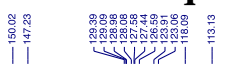

$3 q$

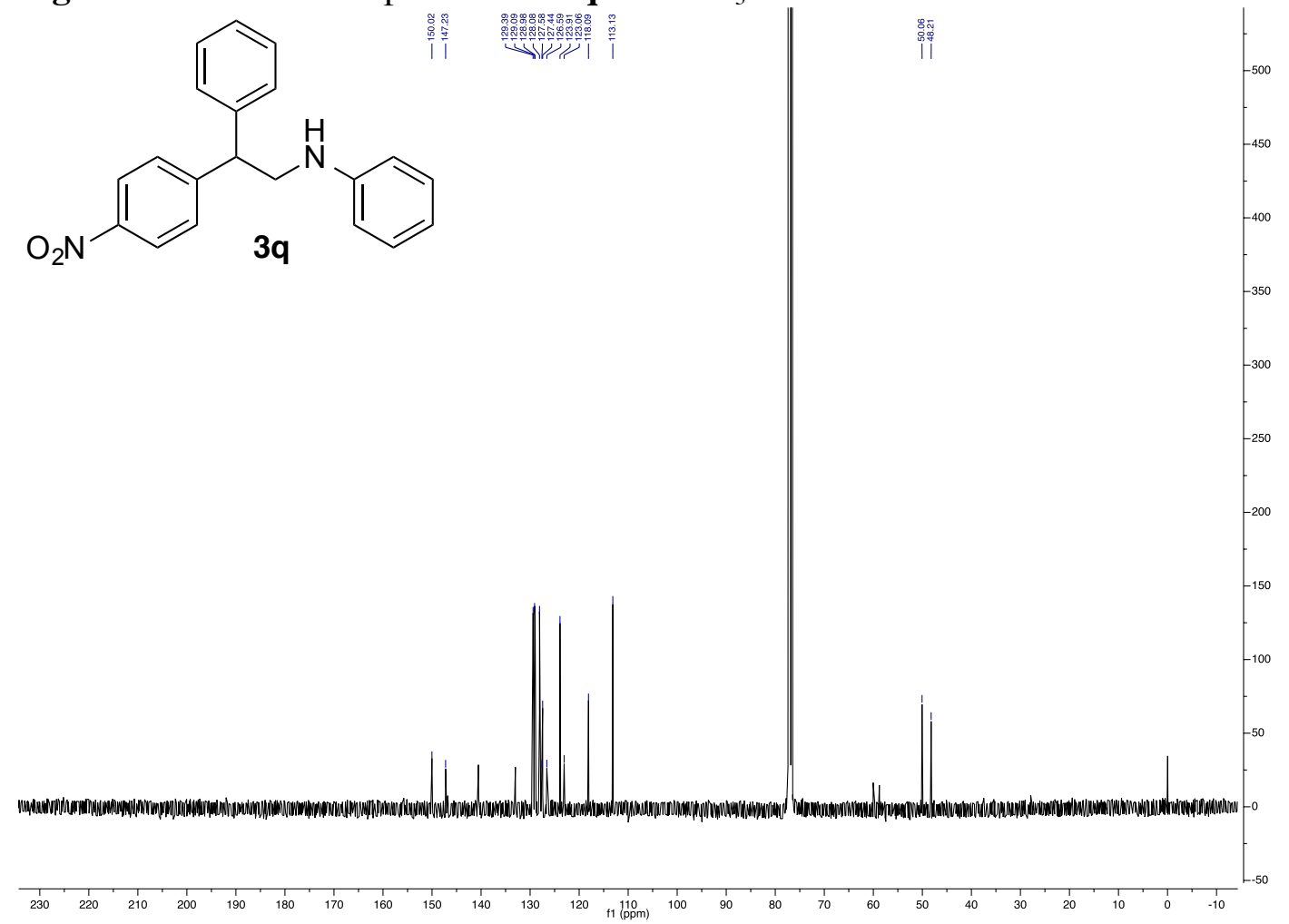

率 
Figure S29: ${ }^{1} \mathrm{H}-\mathrm{NMR}$ spectrum of crude $\mathbf{3 a}$ in $\mathrm{CDCl}_{3}$ with 1,3,5-trimethoxybenzene as internal standard.<smiles>c1ccc(NCC(c2ccccc2)c2ccccc2)cc1</smiles>

3a
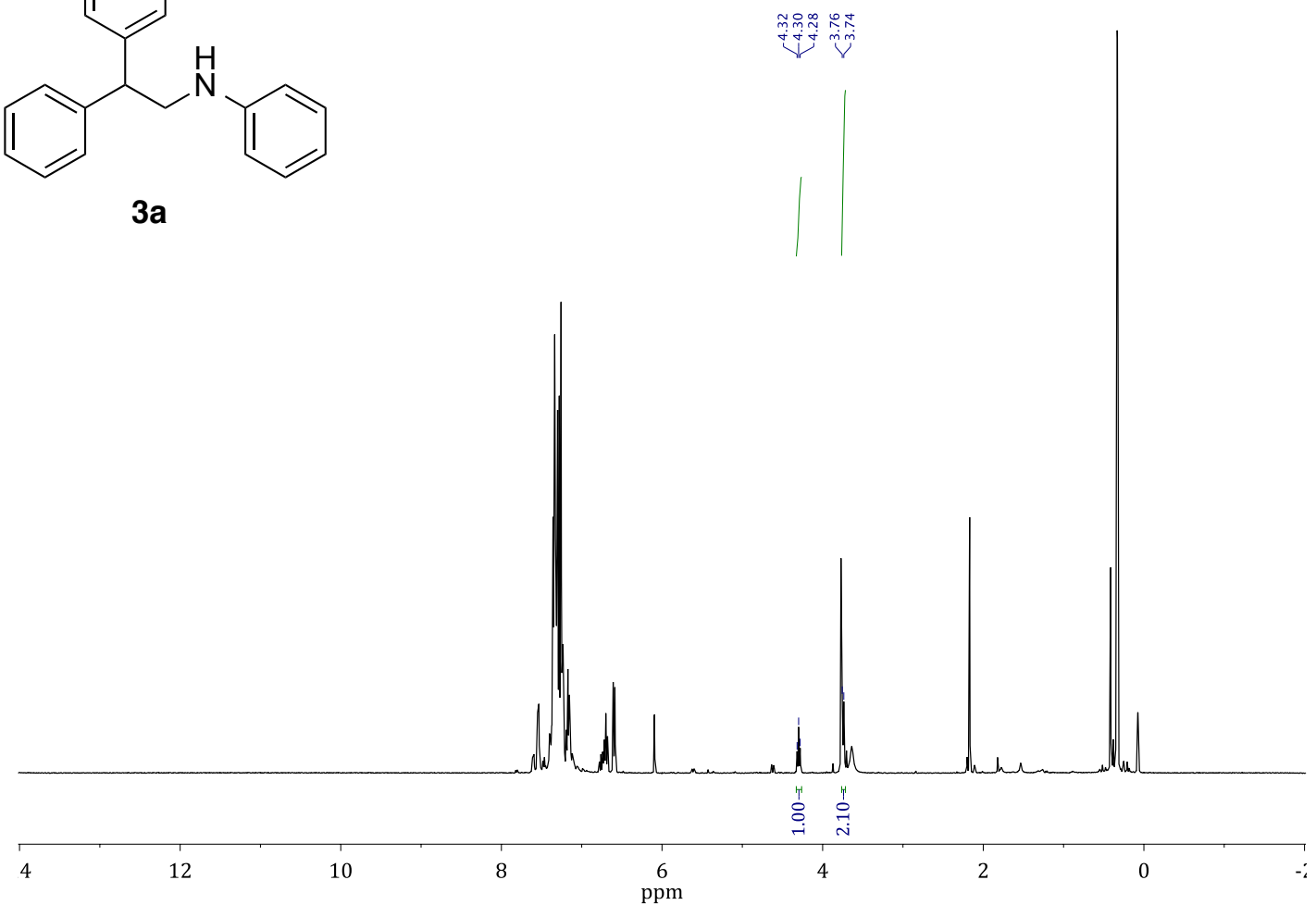

Figure S30: ${ }^{1} \mathrm{H}-\mathrm{NMR}$ spectrum of crude $3 \mathbf{r}$ in $\mathrm{CDCl}_{3}$ with 1,3,5-trimethoxybenzene as internal standard.

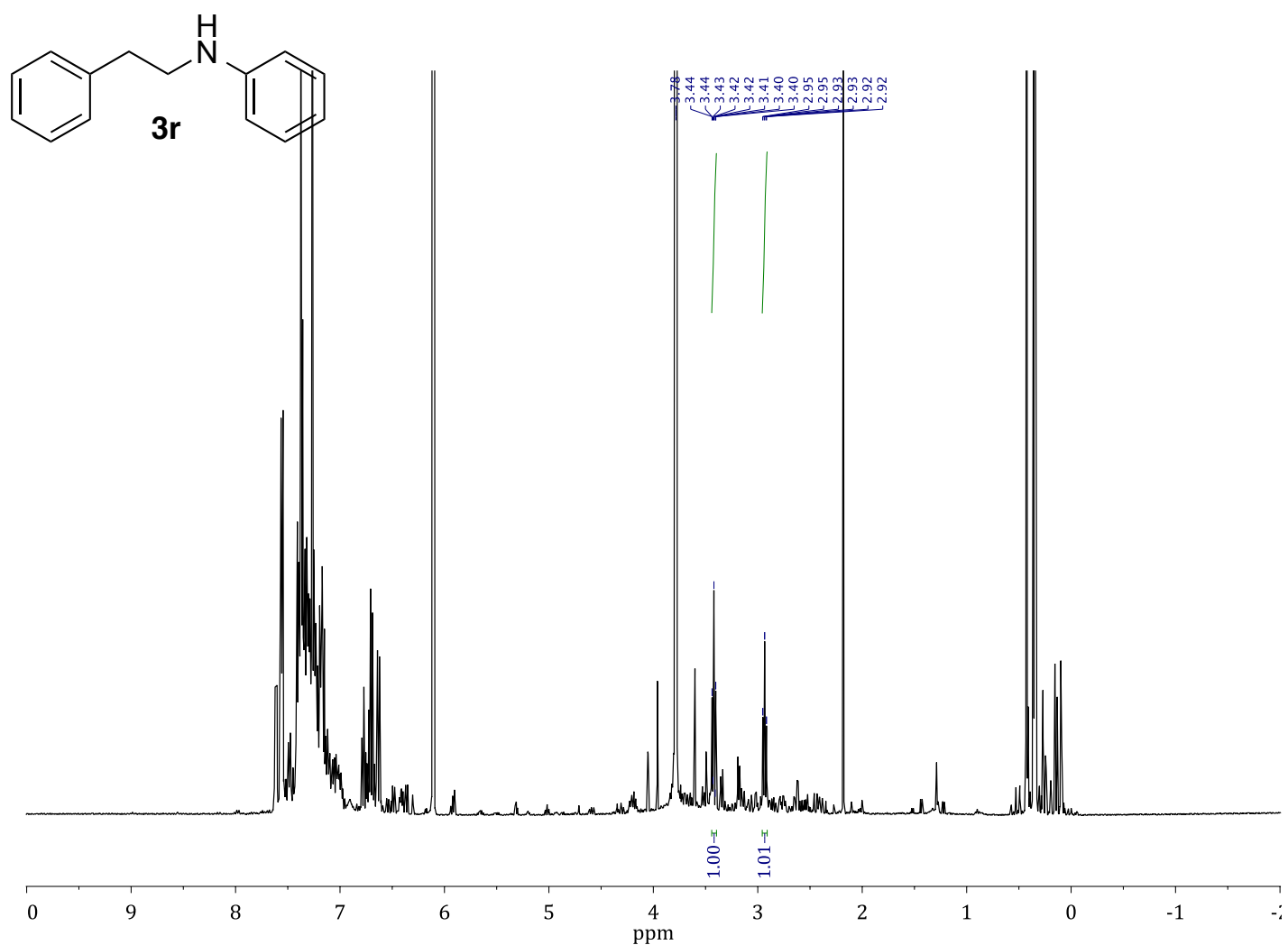


Figure S31: ${ }^{1} \mathrm{H}-\mathrm{NMR}$ spectrum of crude $3 \mathrm{~s}$ in $\mathrm{CDCl}_{3}$ with 1,3,5-trimethoxybenzene as internal standard.<smiles>Clc1ccc(CCNc2ccccc2)cc1</smiles>
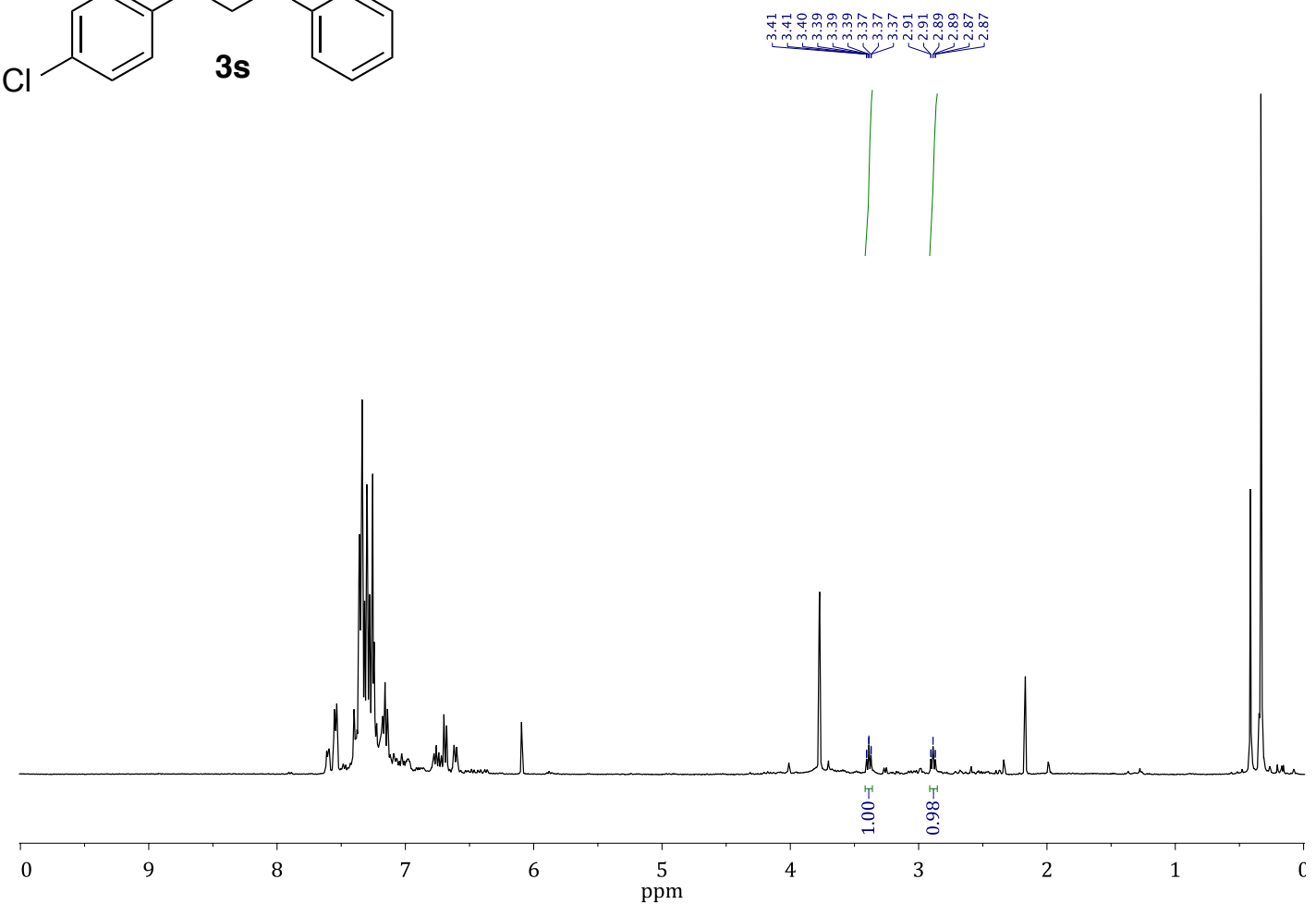

Figure S32: ${ }^{1} \mathrm{H}-\mathrm{NMR}$ spectrum of crude $3 \mathrm{t}$ in $\mathrm{CDCl}_{3}$ with mesitylene as internal standard.<smiles>CC(CNc1ccccc1)c1ccc(C(C)(C)C)cc1</smiles>

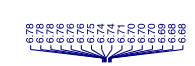

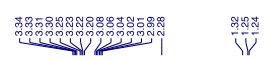
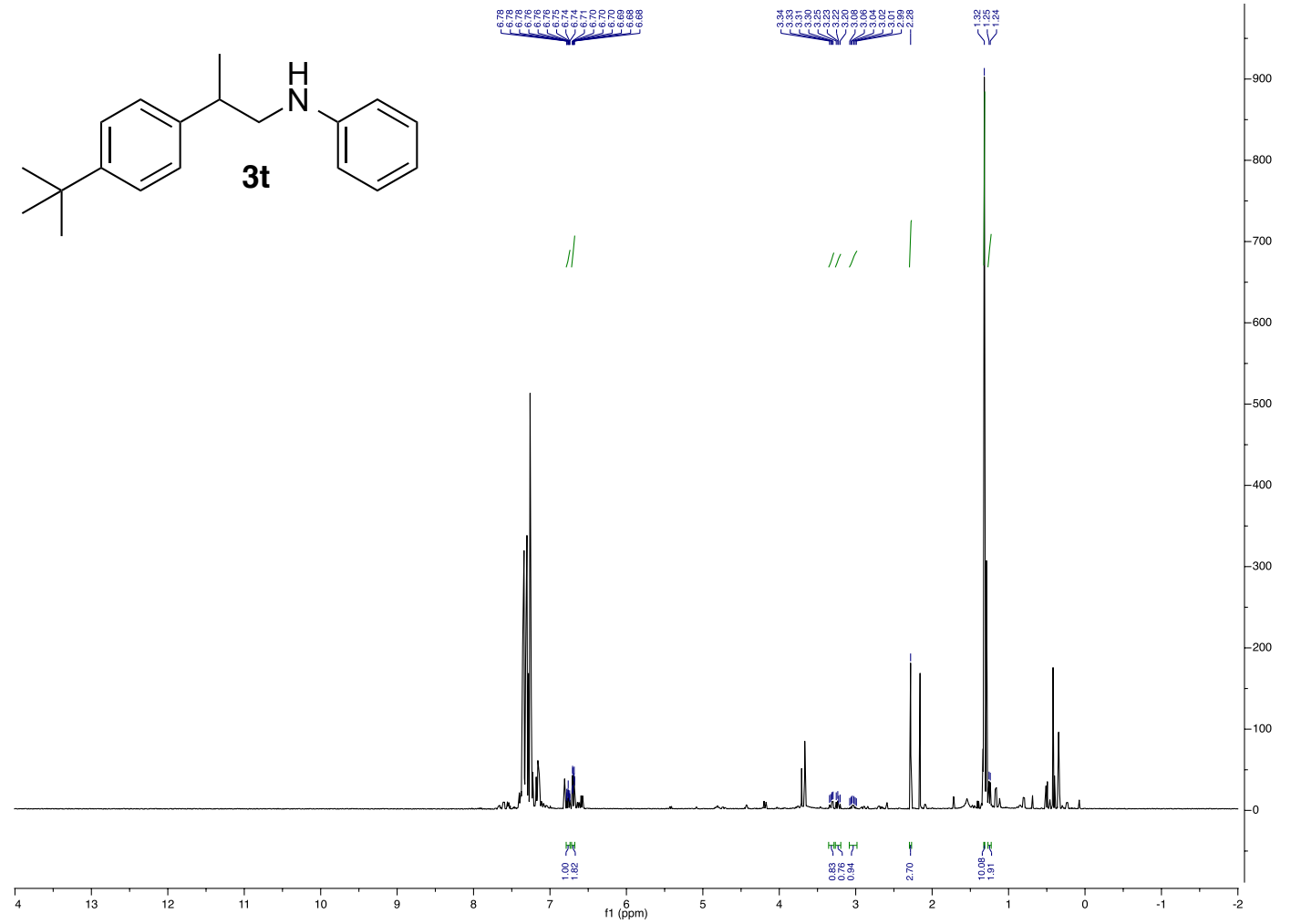
Figure S33: ${ }^{1} \mathrm{H}-\mathrm{NMR}$ spectrum of $\mathbf{S 3}$ in $\mathrm{CDCl}_{3}$.<smiles>CC(C)(C)c1ccc(C=Cc2ccccc2)cc1</smiles>
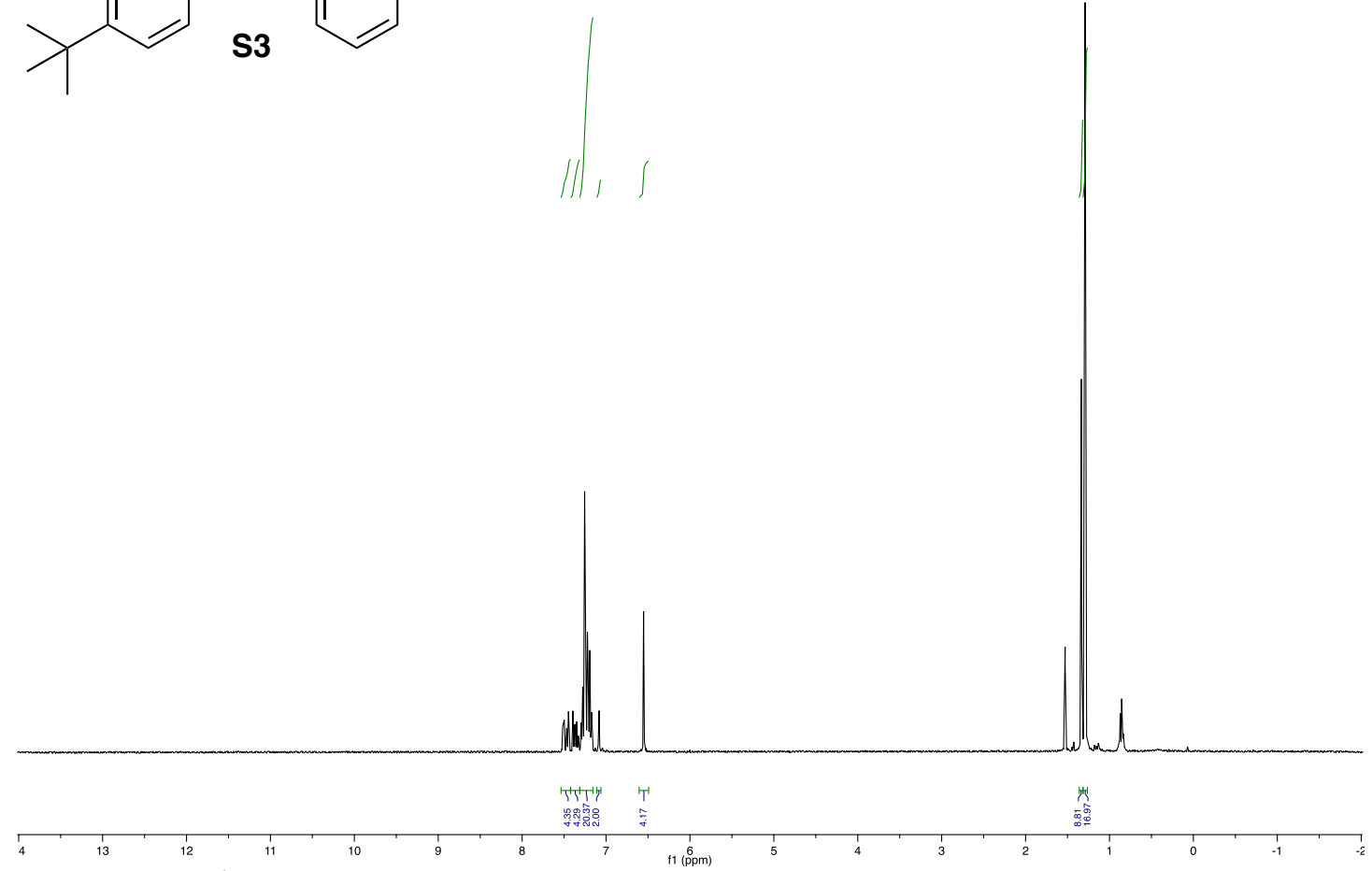

Figure S34: ${ }^{1} \mathrm{H}-\mathrm{NMR}$ spectrum of $\mathbf{1 b}$ in $\mathrm{CDCl}_{3}$.<smiles>CC(C)(C)c1ccc(C2OC2c2ccccc2)cc1</smiles><smiles>[C+]=[13CH]</smiles>

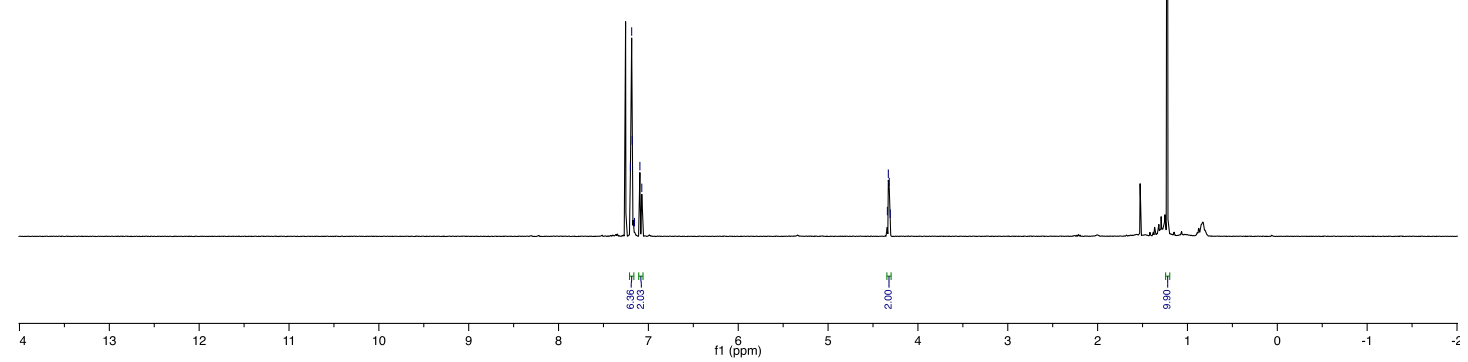


Figure S35: ${ }^{13} \mathrm{C}$-NMR spectrum of $\mathbf{1 b}$ in $\mathrm{CDCl}_{3}$.
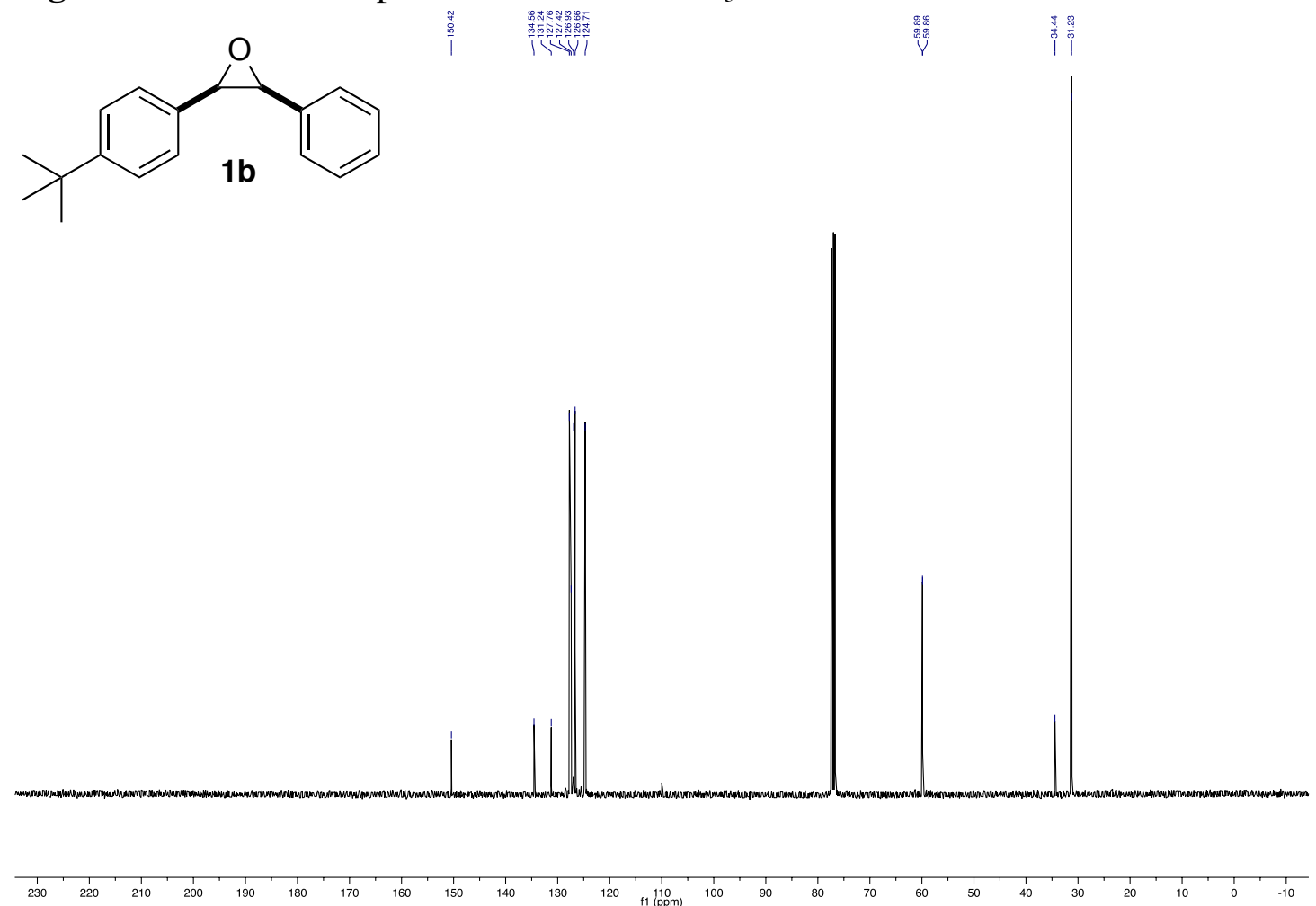

Figure S36: ${ }^{1} \mathrm{H}-\mathrm{NMR}$ spectrum of $1 \mathrm{c}$ in $\mathrm{CDCl}_{3}$.<smiles>Brc1ccc(C2OC2c2ccccc2)cc1</smiles>

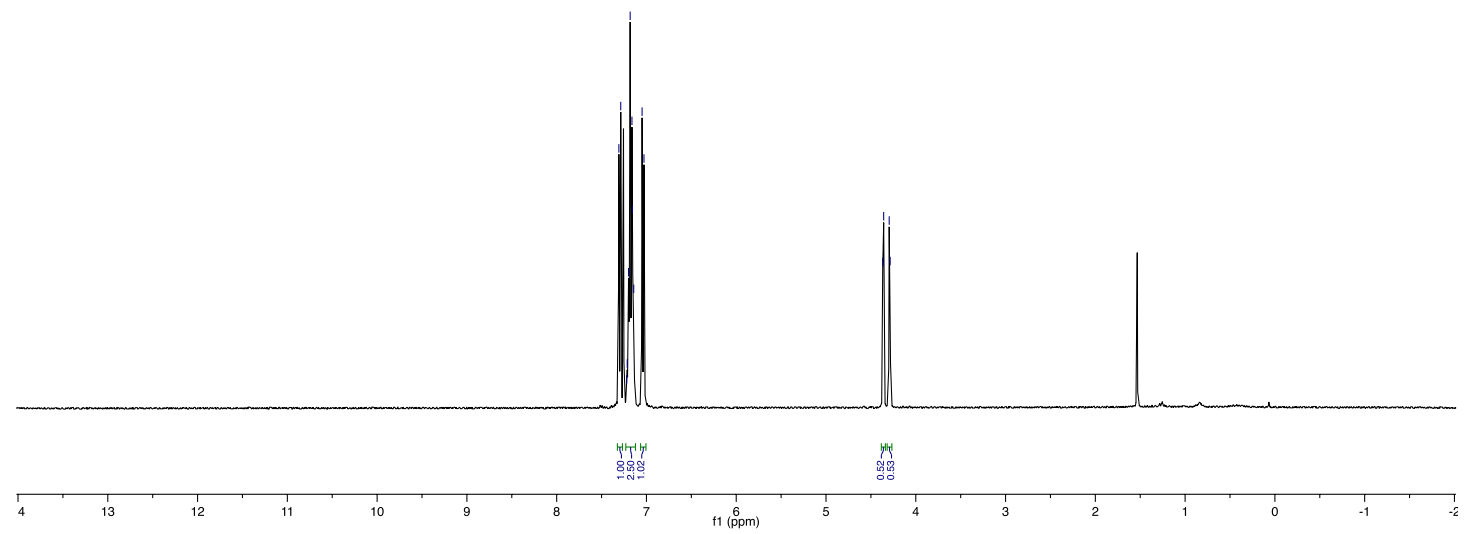


Figure S37: ${ }^{13} \mathrm{C}-\mathrm{NMR}$ spectrum of $1 \mathrm{c}$ in $\mathrm{CDCl}_{3}$.
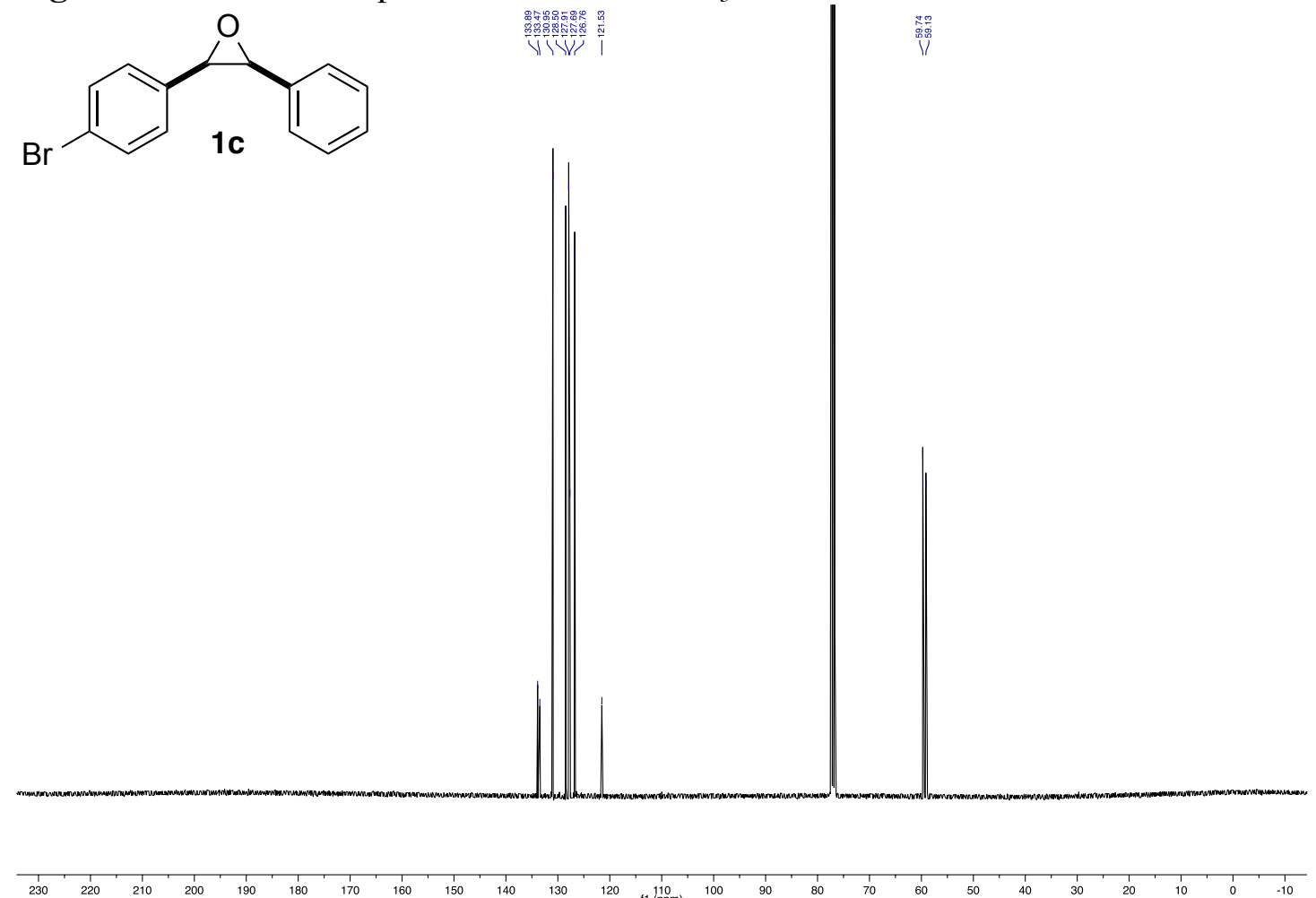

Figure S38: ${ }^{1} \mathrm{H}-\mathrm{NMR}$ spectrum of $\mathbf{1 d}$ in $\mathrm{CDCl}_{3}$.<smiles>Clc1ccc(C2OC2c2ccccc2)cc1</smiles>

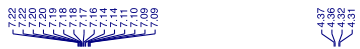

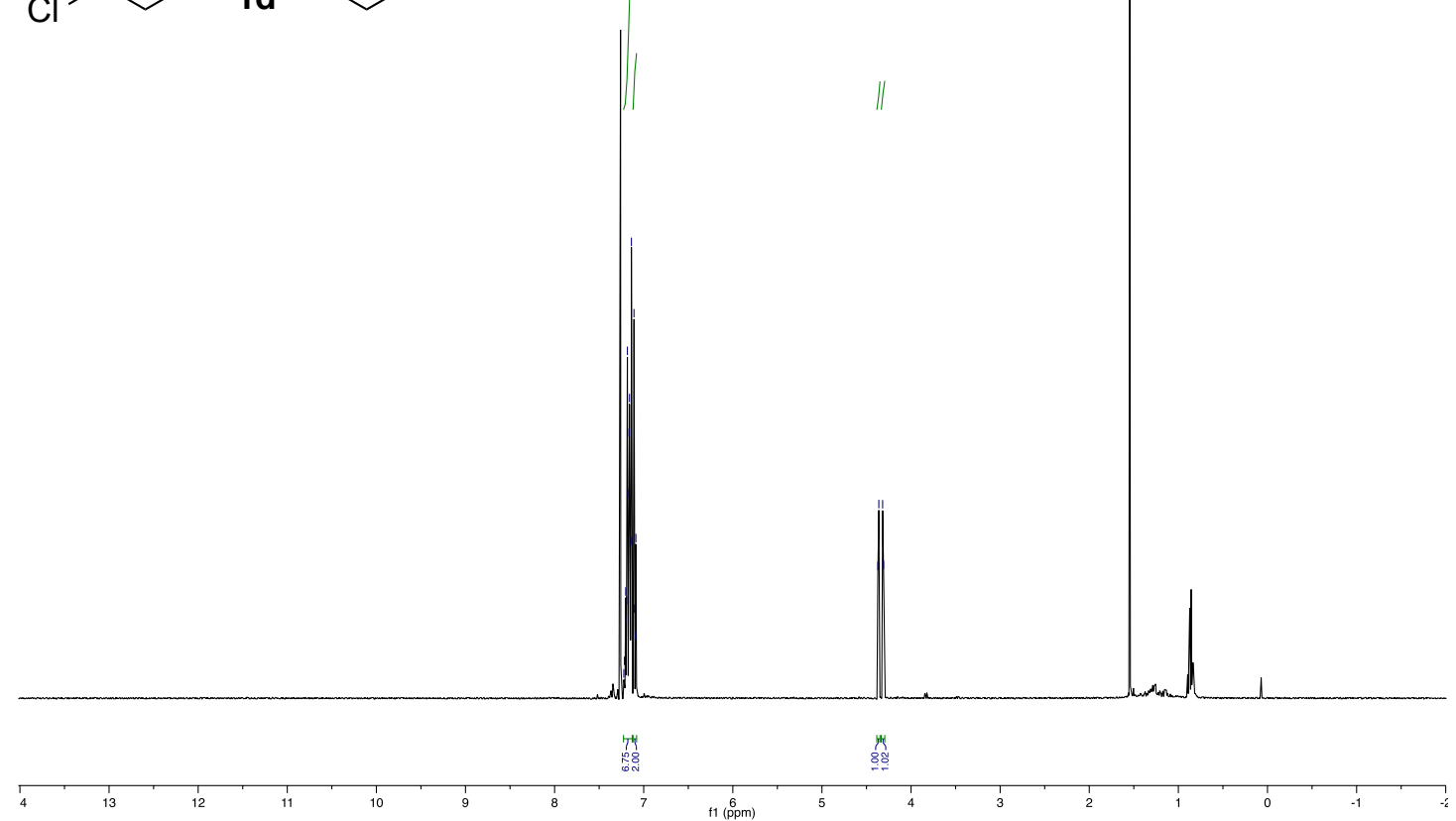


Figure S39: ${ }^{1} \mathrm{H}-\mathrm{NMR}$ spectrum of $1 \mathrm{e}$ in $\mathrm{CDCl}_{3}$.

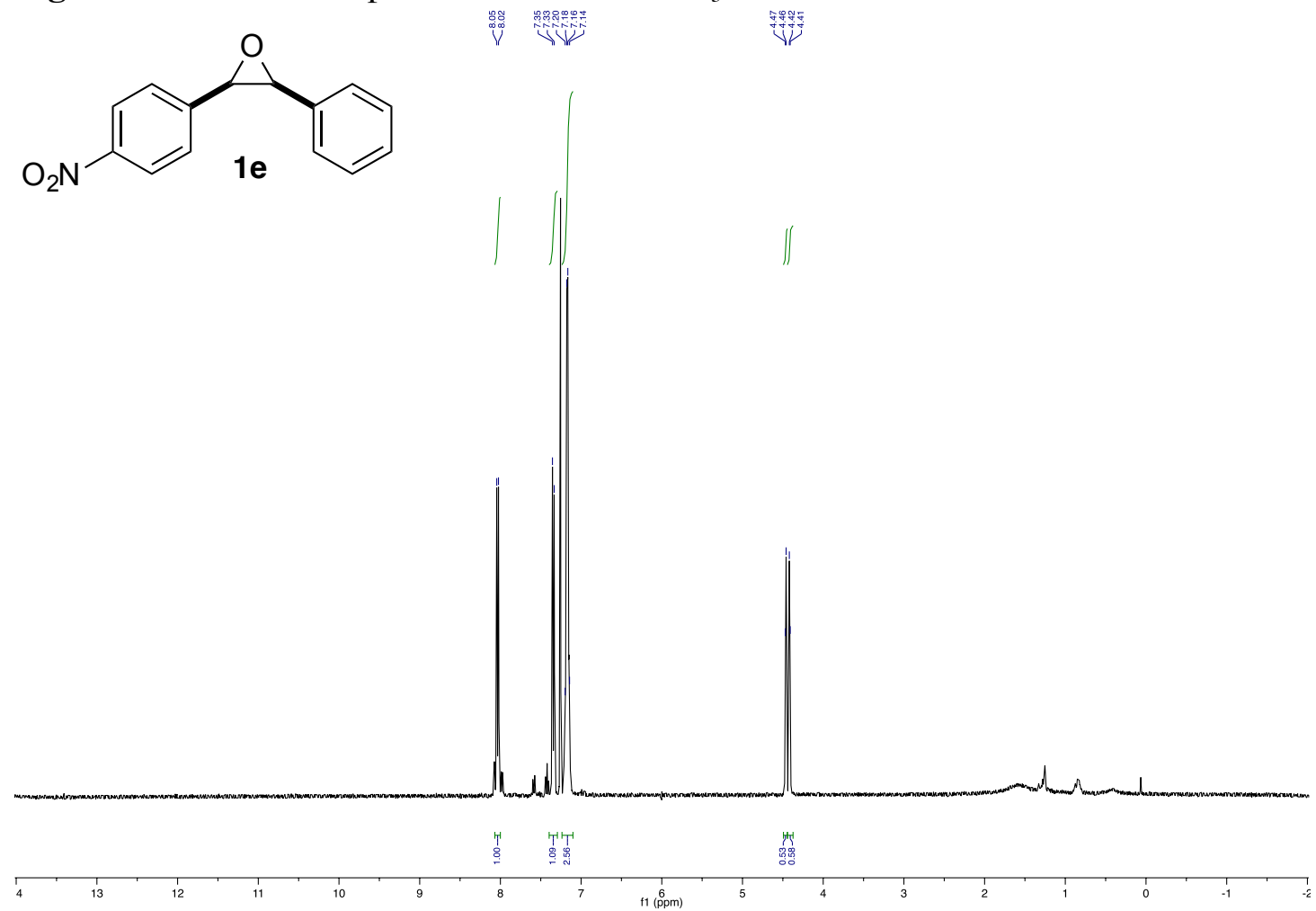

Figure S40: ${ }^{13} \mathrm{C}-\mathrm{NMR}$ spectrum of $1 \mathrm{e}$ in $\mathrm{CDCl}_{3}$.
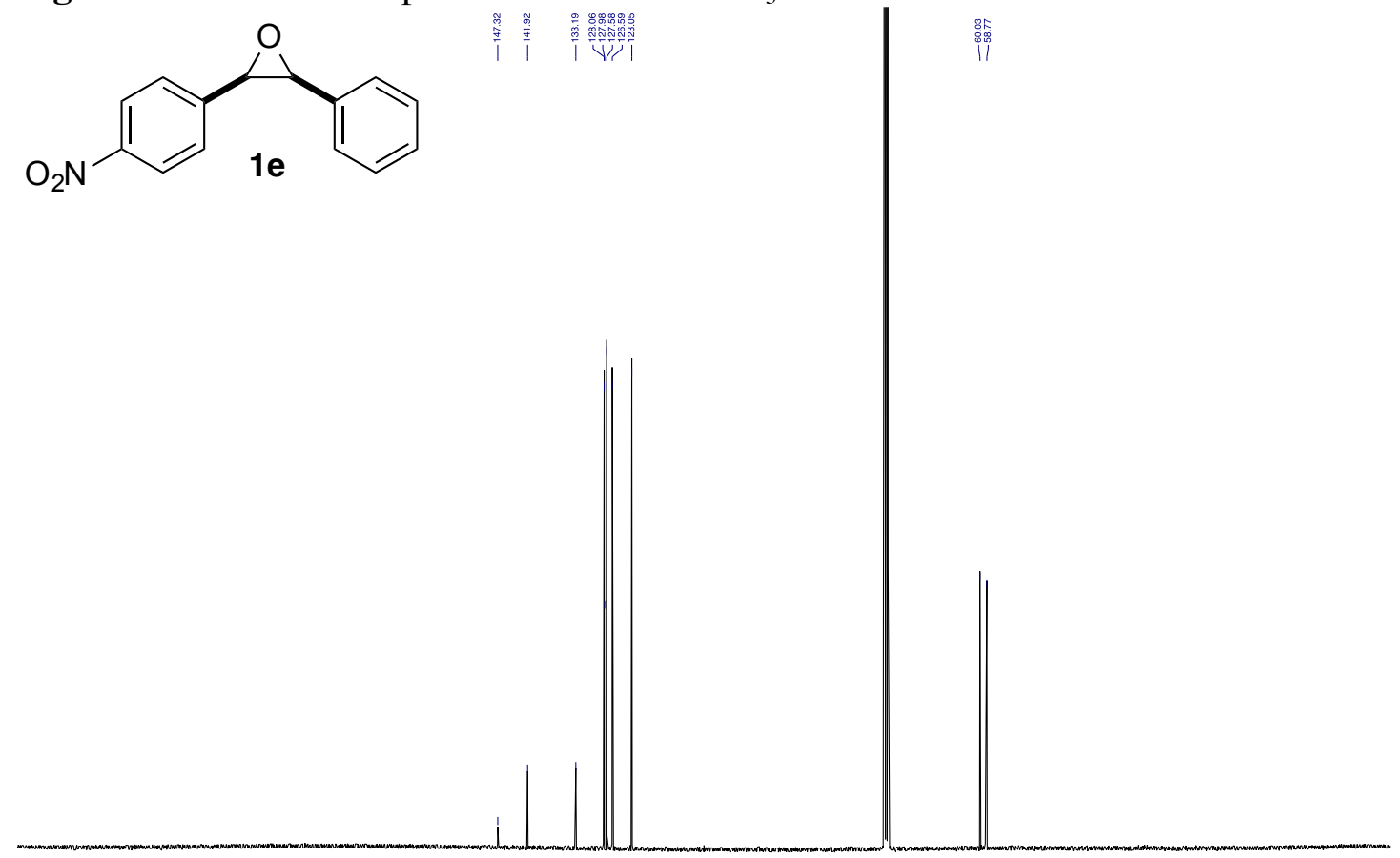
Figure S41: ${ }^{1} \mathrm{H}-\mathrm{NMR}$ spectrum of $\mathbf{S 1 1}$ in $\mathrm{CDCl}_{3}$.<smiles>CC(C)(C)c1ccc(C=CCS)cc1</smiles><smiles></smiles>

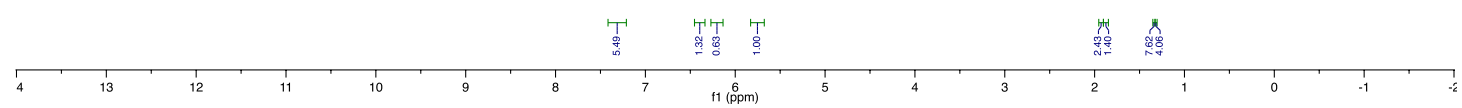

Figure S42: ${ }^{13} \mathrm{C}-\mathrm{NMR}$ spectrum of $\mathbf{S 1 1}$ in $\mathrm{CDCl}_{3}$.<smiles>C/C=C\c1ccc(C(C)(C)C)cc1</smiles>

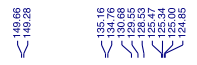

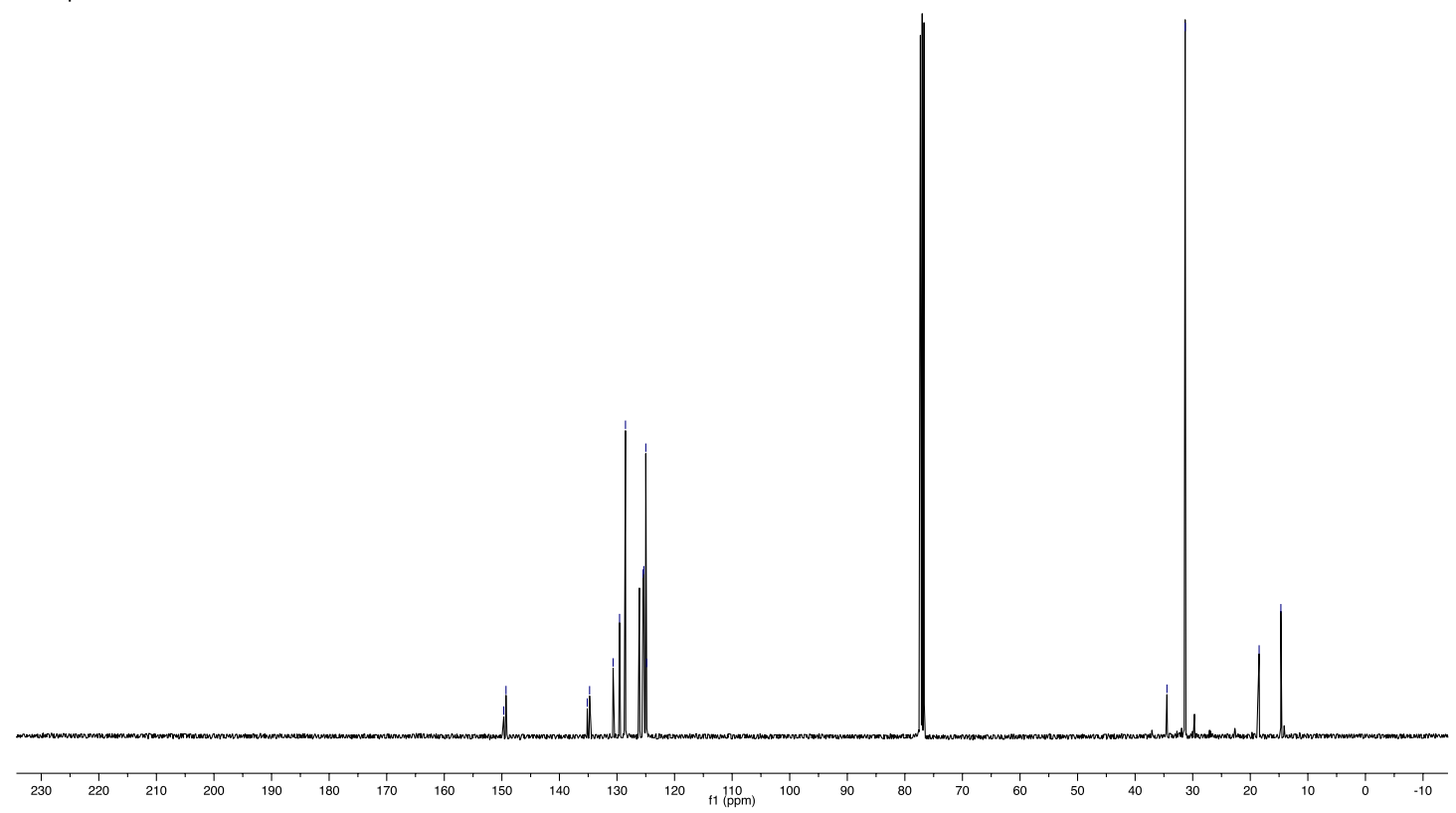


Figure S43: ${ }^{1} \mathrm{H}-\mathrm{NMR}$ spectrum of $3 \mathbf{i}$ in $\mathrm{CDCl}_{3}$.<smiles>CC1OC1c1ccc(C(C)(C)C)cc1</smiles>

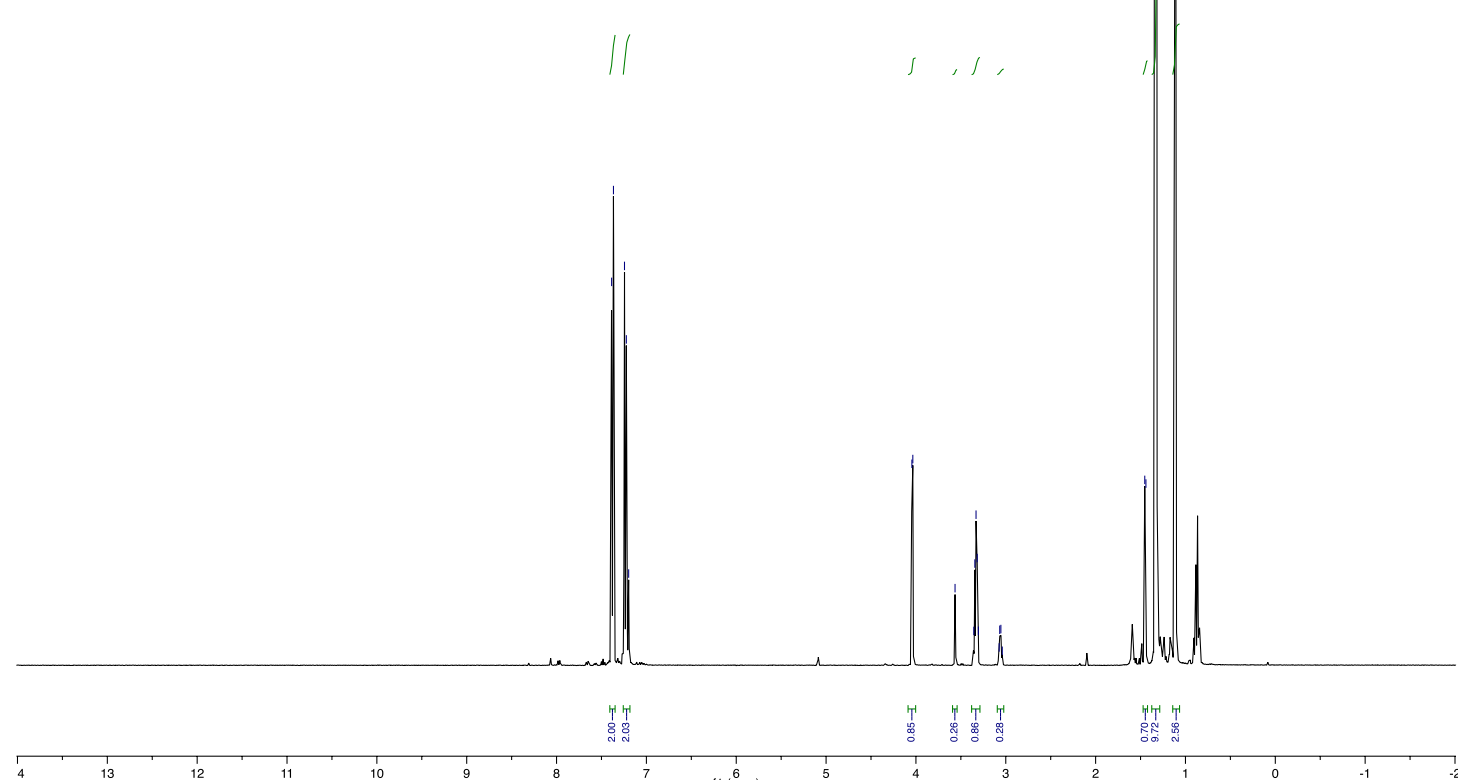

Figure S44: ${ }^{13} \mathrm{C}-\mathrm{NMR}$ spectrum of $3 \mathrm{i}$ in $\mathrm{CDCl}_{3}$.
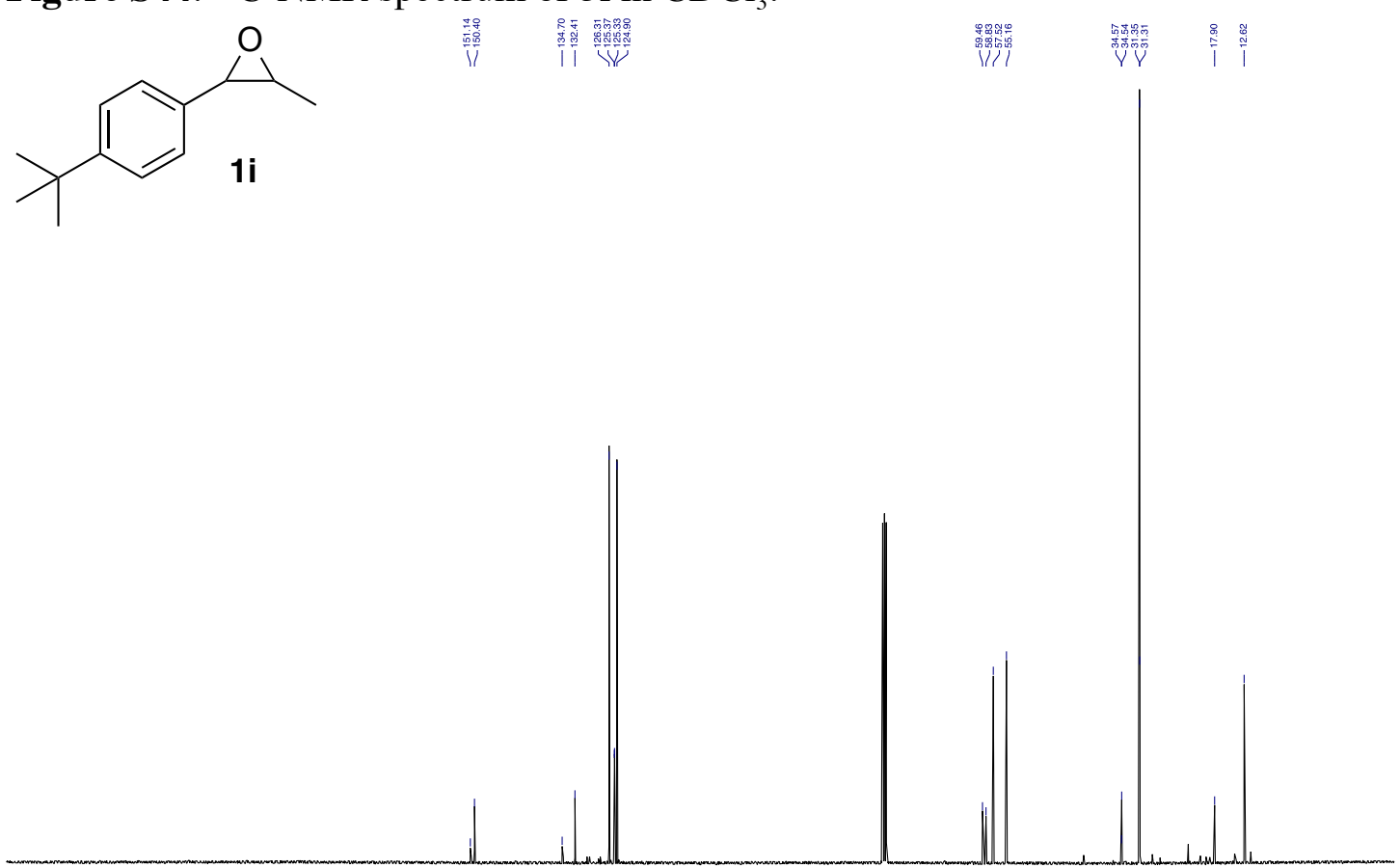
Figure S45: ${ }^{1} \mathrm{H}-\mathrm{NMR}$ spectrum of $\mathrm{B}(p \text {-tol })_{3}$.

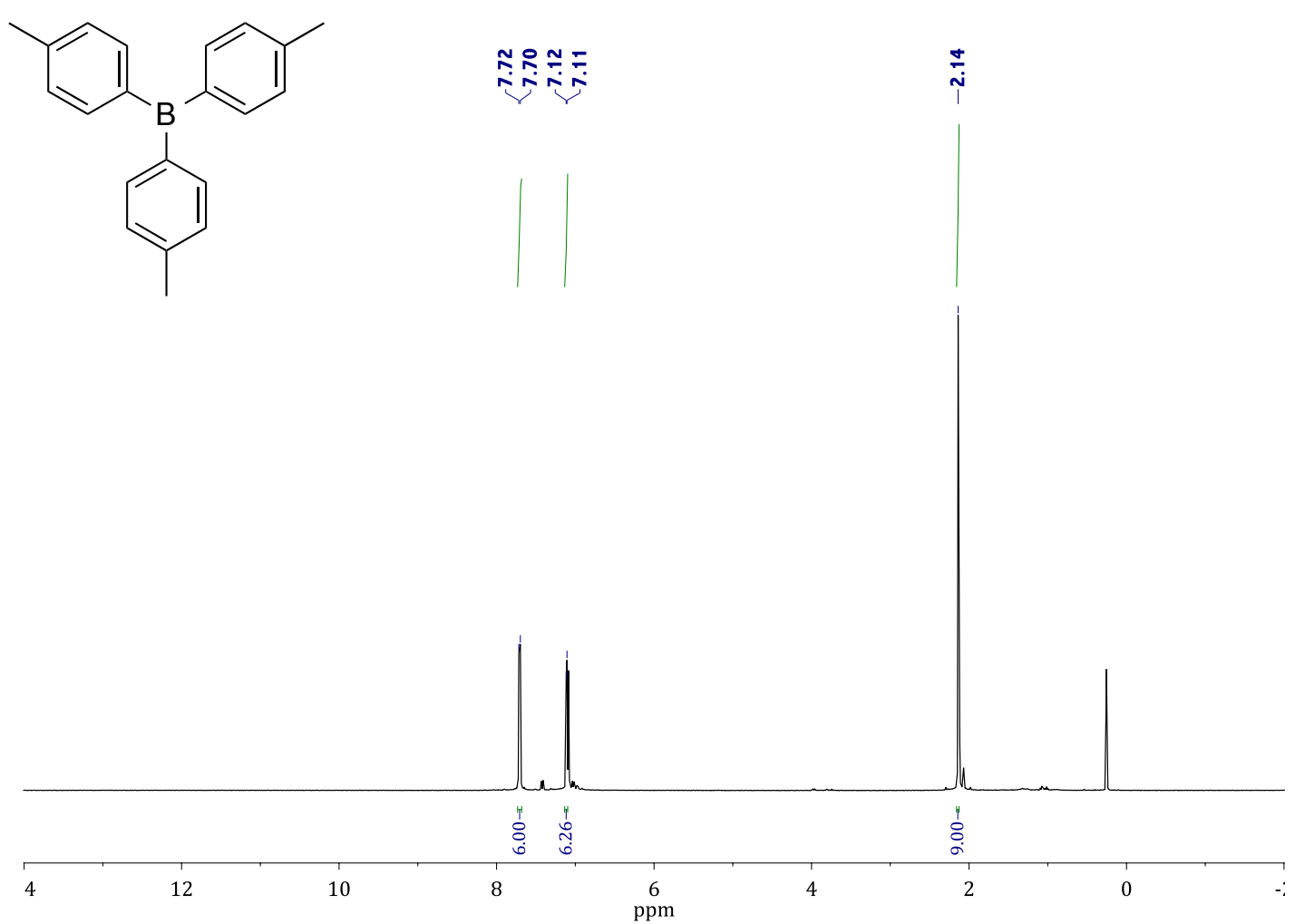

